\title{
Real time cAMP dynamics in the vicinity of phospholemman in healthy and failing cardiomyocytes
}

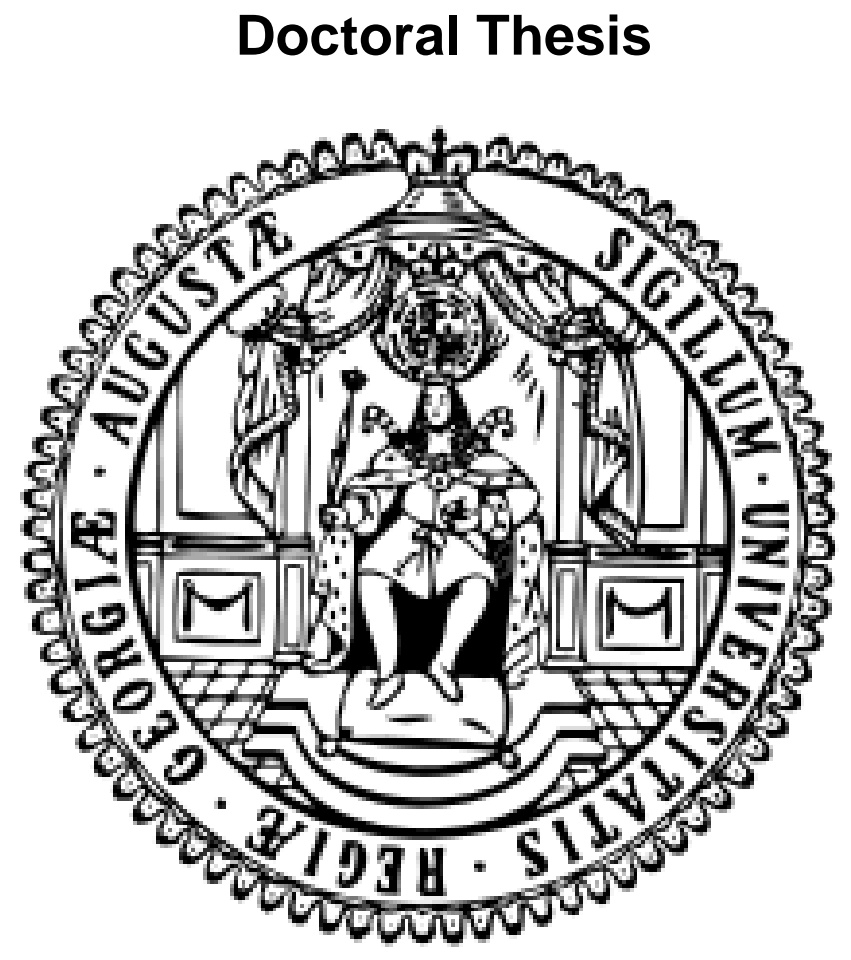

In partial fulfillment of the requirements for the degree "Doctor of Philosophy (Ph.D.)" in the Molecular Medicine Study Programme at the Georg-August University Göttingen

submitted by Zeynep Baştuğ-Özel born in Kassel, Germany 


\section{Dedication}

This dissertation is dedicated

to my uncle Prof. Turgut Baştuğ, who always inspired me by broadening my horizons

and

to my beloved parents Hülya and llyas Baştuğ for their endless support throughout my life. 


\section{Members of the Thesis Committee}

First member of the thesis committee/ supervisor:

Prof. Dr. Viacheslav Nikolaev

Institute of Experimental Cardiovascular Research,

University Medical Centre, UKE, Hamburg

Second member of the thesis committee:

Prof. Dr. Blanche Schwappach

Department of Molecular Biology

University Medical Centre, UMG, Goettingen

Third member of the thesis committee:

Prof. Dr. Walter Stühmer

Department Molecular Biology of Neuronal Signals

Max-Planck-Institute for Experimental Medicine, MPI, Goettingen

Date of Disputation: $15^{\text {th }}$ July 2016 


\section{$\underline{\text { Affidavit }}$}

Here I declare that my doctoral thesis entitled

"Real time cAMP dynamics in the vicinity of phospholemman in healthy and failing cardiomyocytes"

has been written independently with no other sources and aids than quoted.

\section{Zeynep Baştuğ-Özel}

Göttingen, June 2016 


\section{List of publications}

Book chapter:

Zeynep Bastug and Viacheslav O. Nikolaev. Monitoring Real-Time Cyclic Nucleotide Dynamics in Subcellular Microdomains, Chapter 8: 135-146. Cyclic Nucleotide Signaling (Methods in Signal Transduction Series) edited by Xiaodong Cheng, May 12, 2015 by CRC Press. ISBN 9781482235562.

Manuscript in preparation:

Zeynep Bastug-Özel, Peter Wright, Davor Pavlovic, Jacqueline Howie, William Fuller, Julia Gorelik, Michael Shattock and Viacheslav O. Nikolaev. Heart failure leads to altered local $\beta_{2^{-}}$ adrenoceptor/cAMP dynamics in the vicinity of phospholemman. 2016 


\section{$\underline{\text { Table of contents }}$}

List of Abbreviations

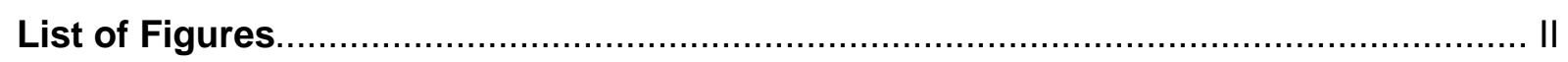

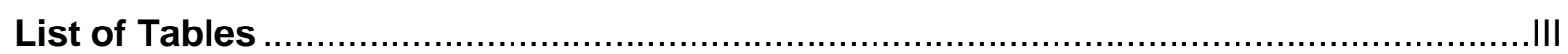

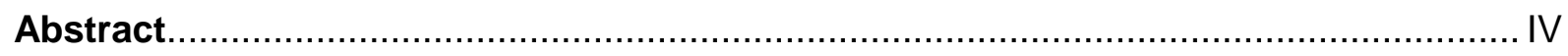

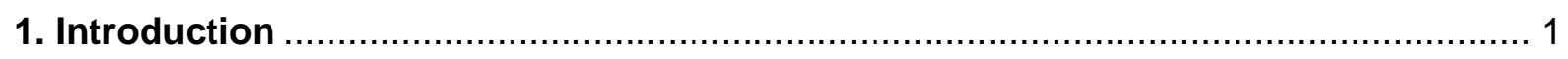

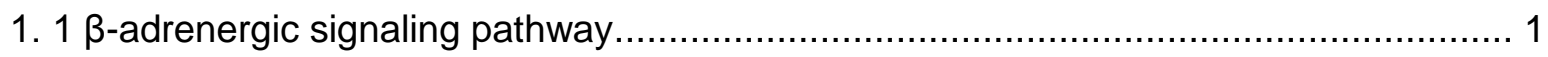

1. 1. 1 Subtype specific $\beta$-adrenergic signaling in the heart .................................... 1

1. 1. 2 CAMP - one second messenger with several functions ................................... 4

1. 1. 3 cAMP compartmentation in the heart - role of Phosphodiesterases and AKAPs.. 5

1. 1. 4 Changes of local cAMP signaling and $\beta$-AR subtype modules in cardiac

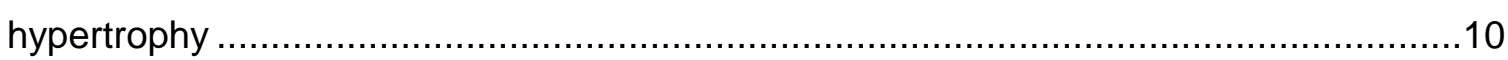

1. 2 Differential regulation of the Na/K-ATPase by Phospholemman in the microdomain..12

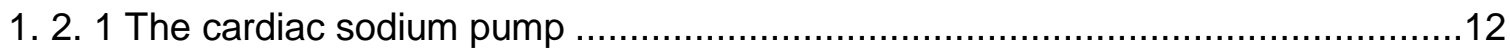

1. 2. 2 Cardiac subunit expression and composition of the Na pump............................13

1. 3 Kinase mediated control of Phospholemman over the Na/K-ATPase ........................14

1. 3. 1 Phospholemman- FXYD1 Protein .............................................................

1. 3. 2 Kinase phosphorylation of Phospholemman …………...................................15

1. 4 Changes of PLM/ NKA complex in diseased heart .............................................17

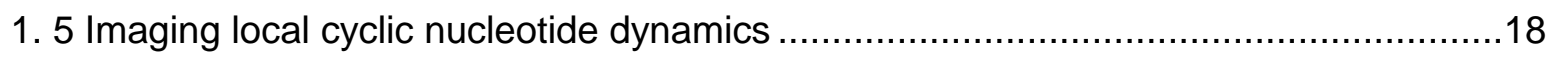

1. 5. 1 Fundamentals of Förster/Fluorescence resonance energy transfer ...................18

1. 5. 2 cAMP sensitive FRET Biosensors ………………......................................19

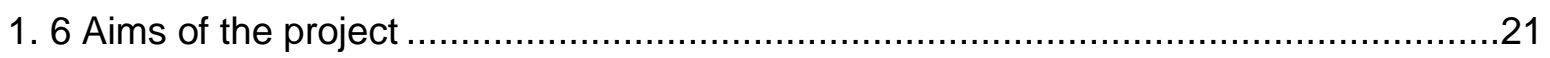

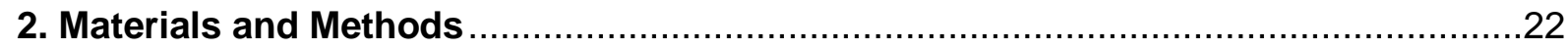

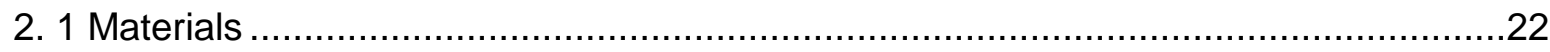

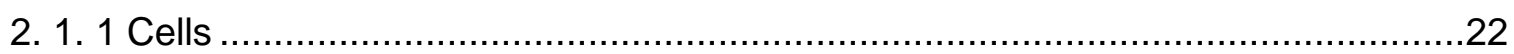

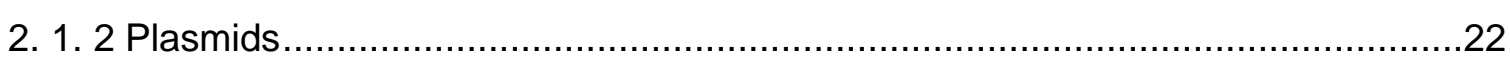

2. 1. 3 Bacteria strains

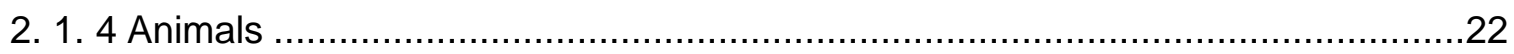

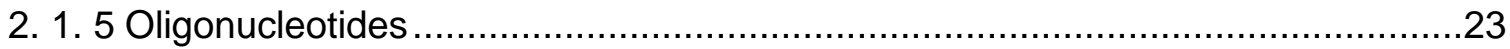

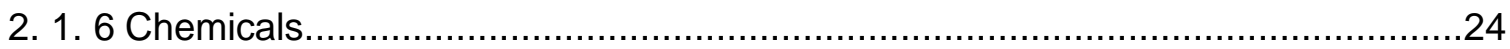

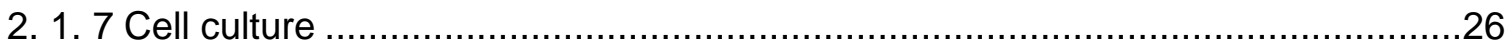




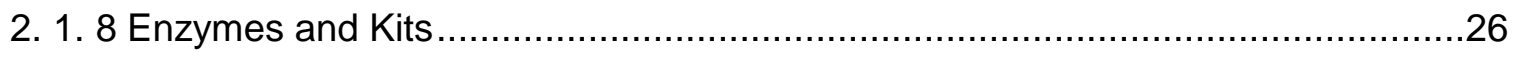

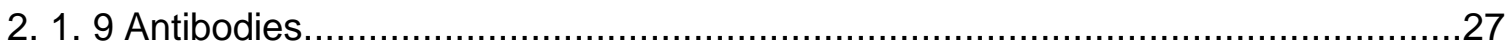

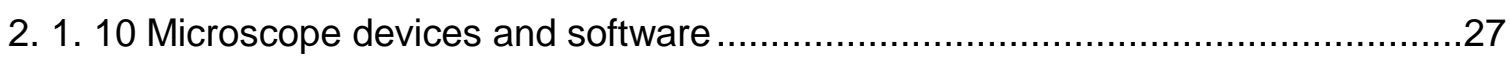

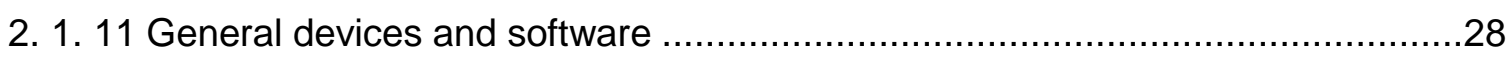

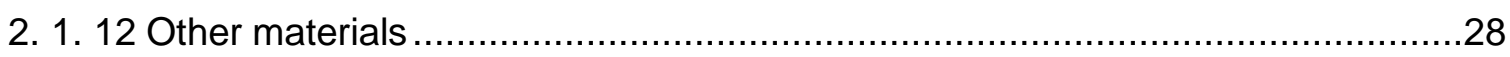

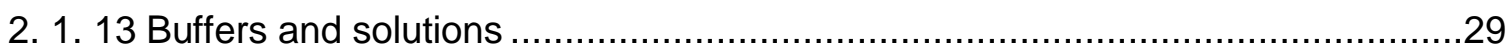

2. 2 Methods

2. 2. 1 Generation of the recombinant plasmids and adenoviral construction of E1-camps and PLM-E1 biosensors .31

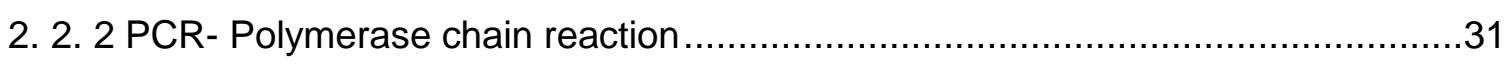

2. 2. 3 Gel electrophoresis, DNA purification and quantification..................................

2. 2. 4 Gel electrophoresis, DNA purification and quantification....................................

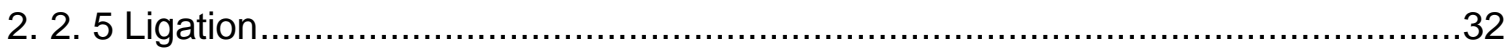

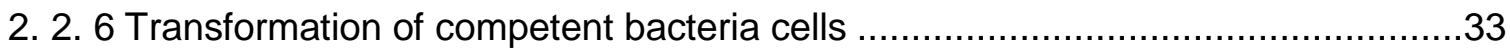

2. 2. 7 Cloning the humanPLM-E1 FRET biosensor based on the PCDNA3 Epac1-camps

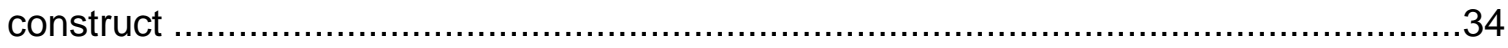

2. 2. 8 Cloning the canine PLM-E1 FRET biosensor based on the PLM-YFP construct .....34

2. 2. 9 Generation of the adenoviral constructs Epac1-camps, human PLM-E1 and

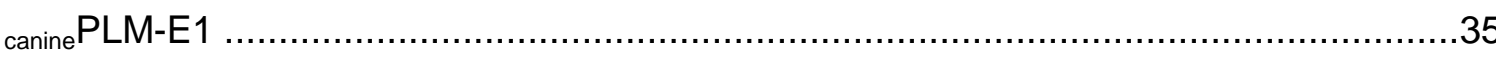

2. 2. 10 Calculating the multiplicity of infection and adenoviral transduction of ARVM ...37

2. 2. 11 Cell culture and transfection of the HEK293A cell line .....................................37

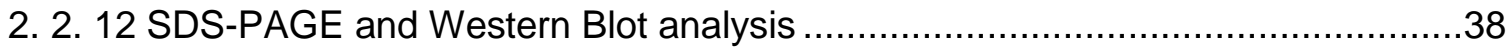

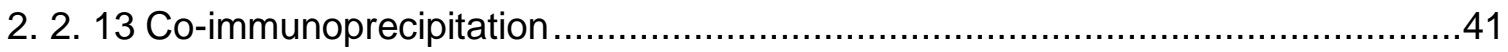

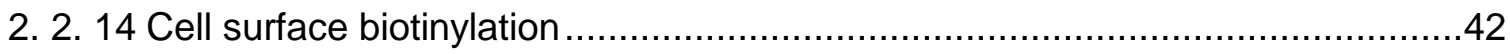

2. 2. 15 Generation of a HEK293A cell line stably expressing PLM-E1 .........................42

2. 2. 16 Ouabain sensitive ${ }^{86} \mathrm{Rb}$ uptake measurements ............................................43

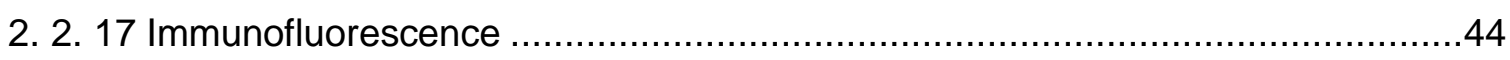

2. 2. 18 Heart failure rat model: Myocardial Infarction................................................44

2. 2. 19 ARVM Isolation by Langendorff perfusion.....................................................44

2. 2. 20 FRET measurements of ARVMs transduced with PLM-E1 and E1-camps.......46

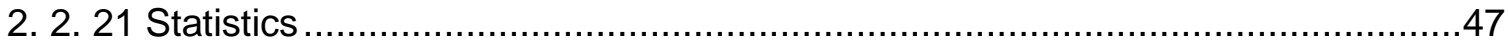

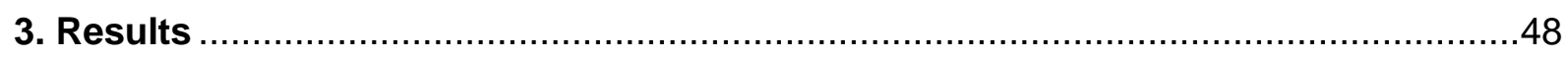

3. 1 Generation of the first version of the PLM-E1 FRET Biosensor ............................48

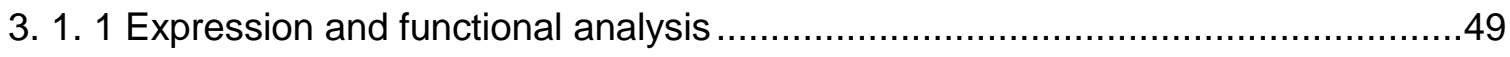

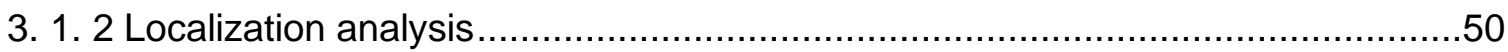


3. 2 Generation of the optimized PLM-E1 FRET Biosensor and the adenoviral construct .51

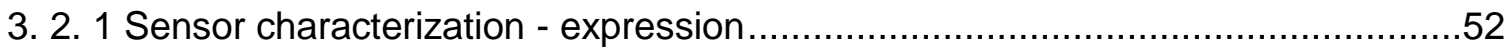

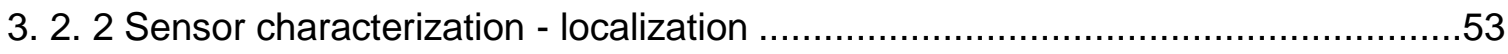

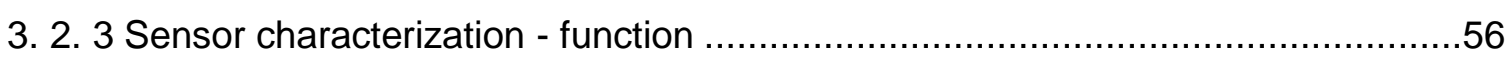

3. 3 Comparative FRET analysis of E1-camps and PLM-E1 transduced ARVMs .............58

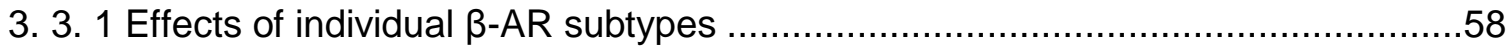

3. 3. 2 PDE Profiles in PLM-E1 and E1-camps transduced ARVMs.............................59

3. 3. 3 ANP/cGMP mediated PDE3 response in the PLM/NKA microdomain.................61

3. 3. 4 FRET measurements in PLM-E1 and E1-camps transfected ARVMs ................63

3. 3. 5 Alterations in local PDE dependent cAMP regulation in the PLM/NKA microdomain

3. 3. 6 cAMP degrading PDE3 pools in the PLM/NKA microdomain are delocalized in the hypertrophied heart .65

4. Discussion

4. 1 Challenges in generating novel PLM-E1 FRET biosensors with correct structural and functional properties.

4. 2 Comparative FRET analysis of healthy ARVMs expressing adenoviral PLM-E1 and

E1-camps biosensors reveal differentially regulated local $\beta$-adrenergic signaling 69

4. 2. 1 Restricted cAMP diffusion to the NKA/PLM complex via basal activity of phosphodiesterases

4. 2. 2 Locally confined $\beta_{2}$-AR mediates cAMP signaling pathway proximal to the PLM/NKA complex . .70

4. 2. 3 Critical PDE3-dependent regulation of $\beta_{2}-A R$ mediated cAMP in the vicinity of PLM.

4. 3. Alterations of $\beta_{2}-A R$ control over subsarcolemmal PLM microdomain through localized PDE2/PDE3 subsets in ARVM with chronic heart failure.

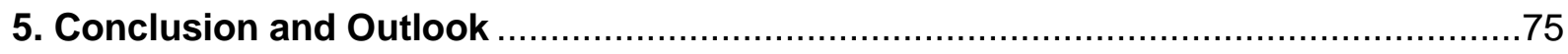

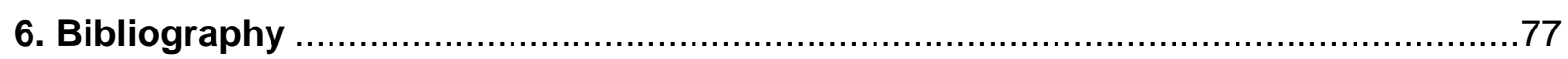

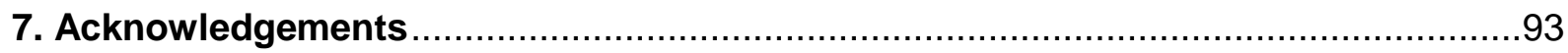




\section{List of Abbreviations}

AC

AKAP

Akt

AMC

ANP

ARVM

Bay

$\beta$-Arr

$\beta_{1}-\mathrm{AR}$

$\beta_{2}-A R$

$\mathrm{Ca}^{2+}$

CaMKII

cAMP

Cav

CFP

cGMP

CGP

CHF

Cilo

CNGC

CREB

ECC

Epac

FRET

for

FXYD

GC

$\mathrm{G}_{\mathrm{i}}$

$\mathrm{G}_{\mathrm{s}}$

GFP

GPCR

GRK

GTPase

HCN2

HEK cell

I-1 adenylyl cyclase

A kinase anchoring protein

protein kinase $B$

aged matched control

atrial natriuretic peptide

adult rat ventricular myocyte

BAY 60-7550

$\beta$-arrestin

$\beta_{1}$-adrenergic receptor

$\beta_{2}$-adrenergic receptor

calcium ion

$\mathrm{Ca}^{2+}$-calmodulin-dependent protein kinase

3',5'-cyclic adenosine monophosphate

caveolin

cyan fluorescent protein

3',5'-cyclic guanosine monophosphate

CGP-20-712A

congestive heart failure

cilostamide

cyclic nucleotide gated channel

cAMP response element binding (protein)

excitation contraction coupling

exchange protein directly activated by cAMP

Förster (fluorescence) resonance energy transfer

forward

consensus motif Proline-Phenylalanine-x-tyrosine-Aspartic acid guanylyl cyclase

inhibitory G-protein

stimulatory G-protein

green fluorescent protein

G-protein coupled receptor

G-protein coupled receptor kinase

guanosine triphosphatase

hyperpolarization-activated cyclic nucleotide-gated channel 2 human embryonic kidney cell

inhibitor-1 
IBMX

ICI

IP3

Iso

$\mathrm{K}^{+}$

$\mathrm{K}_{\mathrm{m}}$

$\mathrm{KO}$

LTCC

$\mathrm{MI}$

$\mathrm{Na}^{+}$

NCX

NKA

NO

PDE

PI3KY

PKA

PKC

PKG

PLM

PLN

PLCE

PP1

Rap

$\mathrm{Rb}$

rev

Roli

RT

RyR2

Ser

SERCA2a

SICM

SR

SU

Thr

Tnl

$V_{\text {max }}$

YFP
3-Isobutyl-1-methylxanthine

ICl 118,551

inositol-1,4,5-triphosphate

isoproterenol/ isoprenaline

potassium ion

Michaelis-Menten constant

knockout

L-type calcium channe I/ $\mathrm{Ca}_{v} 1.2$

myocardial infarction

sodium ion

sodium-calcium exchanger

sodium-potassium ATPase / sodium pump

nitric oxide

phosphodiesterase

phosphoinositide-3 kinase gamma

Protein kinase $A$

Protein kinase $C$

cGMP dependent protein kinase

phospholemman

phospholamban

phospholipase $\mathrm{C}$ epsilon

protein phosphatase 1

Ras-related protein

rubidium

reverse

rolipram

room temperature

ryanodine receptor 2

serine residue

sarcoplasmic/endoplasmic reticulum $\mathrm{Ca}^{2+}$ ATPase $2 \mathrm{a}$

scanning ion conductance microscopy

sarcoplasmic reticulum

subunit

threonine

troponin I

maximal velocity (maximal reaction rate)

yellow fluorescent protein 


\section{List of Figures}

Figure 1. Schematic representation of the cellular cAMP signaling cascade 2

Figure 2. Cartoon describing $\beta_{1} A R s$ and $\beta_{2} A R s$ signaling pathways in cardiomyocytes 3

Figure 3. Cartoon depicting the differential role of cardiac cAMP in confined microdomains leading to differential phosphorylation of PKA targets

Figure 4. A-kinase anchoring proteins (AKAPs) controlling subcellular cAMP microdomains in the heart

Figure 5. Alterations of local cAMP signaling in compensated hypertrophy and heart failure

Figure 6. NKA - an integral player in the 'fight-or-flight' response

Figure 7. Structure of the Na/K-ATPase

Figure 8. Amino acid sequence of currently known mammalian FXYD proteins 15

Figure 9. Phosphorylation of phospholemman regulates sodium pump activity 16

Figure 10. The principles of FRET instancing the CFP and YFP pair 19

Figure 11. Cartoon showing means to determine intracellular cAMP dynamics 20

Figure 12. The co-Immunoprecipitation method $\quad 41$

Figure 13. Layout of the FRET imaging setup $\quad 47$

Figure 14. PLM-E1 construct in pcDNA3.0 vector 48

Figure 15. Expression, function and localization of PLM-E1 in HEK 293 cells 49

Figure 16. Co-immunoprecipitation $\quad 50$

Figure 17. Design of the optimized PLM-E1 biosensor construct 52

Figure 18. Expression of the PLM-E1 sensor 53

Figure 19. Cell surface biotinylation of PLM-E1 transduced ARVM 53

Figure 20. Co-localization of PLM-E1 with the sodium pump 54

Figure 21. Association of PLM-E1 with the sodium-potassium pump a1 subunit 55

Figure 22. Inhibition of NKA activity by PLM-E1 56

Figure 23. Sensor function in PLM-E1 transduced ARVMs 56

Figure 24. Sensitivity of E1-camps and PLM-E1 to adrenergic stimuli 57

Figure 25. Predominant control of $\beta_{2}$-AR over the PLM/NKA microdomain 59

Figure 26. Significant PDE2 contribution to basal cAMP degradation in the vicinity of PLM 60 Figure 27. PDE3 pool in the vicinity of PLM confines $\beta_{2}$ AR mediated cAMP signals to the PLM/NKA microdomain 
Figure 28. Co-localization of the PDE3A isoform with the PLM microdomain

Figure 29. ANP induced PDE3 inhibition in ARVM results in high levels of $\beta_{2}-A R / C A M P$ in the PLM/NKA microdomain

Figure 30. Contributions of individual PDEs to cAMP hydrolysis after $\beta_{2}$-AR stimulation in $\mathrm{AMC}$ and $\mathrm{MI}$ cardiomyocytes

Figure 31. $\beta_{2}$-AR effects in AMC vs. MI cells

Figure 32. Confocal microscopy analysis of PDE3 localization

Figure 33. Cartoon showing highly confined basal cAMP dynamics and a distinct $\beta_{2}$-CAMP effect in the PLM microdomain of ARVMs

Figure 34. Cartoon demonstrating the change in local CAMP dynamics at the cardiac PLM microdomain 


\section{List of Tables}

Table 1. Cardiac PDE enzyme specifications and kinetic properties 6

Table 2. Primary antibodies $\quad 27$

Table 3. Secondary antibodies $\quad 27$

Table 4. Ingredients of the used gels for Western Blot analysis at King's College London 39

Table 5. Ingredients of the used gels for Western Blot analysis in UMG, Göttingen $\quad 40$

Table 6. Phenotyping of aged matched control and 16 weeks post MI hearts 63 


\section{$\underline{\text { Abstract }}$}

The ubiquitous second messenger $3^{\prime}, 5^{\prime}$-cyclic adenosine monophosphate (CAMP) is a crucial regulator of cardiac function and disease. It is known that cAMP signaling is mediated by discrete functional microdomains each containing a specific subset of differentially localized receptors, protein kinases and specific phosphodiesterases (PDEs). Cardiac phosphodiesterases (PDE1-5; PDE8 and 9) hydrolyze cyclic nucleotides, each with different selectivity and affinity for their substrates cAMP and 3',5'-cyclic guanosine monophosphate (cGMP). In one of these microdomains, the phosphorylation of the FXYD1 protein called Phospholemman (PLM), the negative regulator of $\mathrm{Na}^{+} / \mathrm{K}^{+}-\mathrm{ATPase}$ (NKA), via PKA or PKC leads to an increase of NKA activity and its sodium affinity, thereby lowering intracellular sodium levels and hence limiting cardiac inotropy. By obstructing high sodium levels during adrenergic stimulation it simultaneously favors calcium extrusion via the sodium-calcium exchanger (NCX). This mechanism may prevent calcium-overload, hypertrophy and triggered arrhythmias during cardiac stress. Interestingly, PLM expression is known to be altered in cardiomyocytes from postinfarcted rat hearts which results in depressed NKA activity. However, knowledge about the dynamics of CAMP pool coupled to PLM phosphorylation and the interactions of $\beta$-adrenergic receptors ( $\beta$-AR) with individual cardiac PDE subtypes forming this important microdomain as well as their alterations in cardiac disease is insufficient. To investigate these questions, we developed a Förster Resonance Energy Transfer (FRET)-based PLM targeted CAMP biosensor PLM-E1. It can be used to precisely measure CAMP dynamics and to understand the regulatory mechanisms behind a possible local restriction of $\beta-A R$ mediated CAMP signals in the PLM/NKA microdomain of adult rat ventricular myocytes (ARVMs).

Using functional ${ }^{86}$ Rubidium-flux measurements in a PLM-E1 expressing stable HEK cell line, co-immunoprecipitation analysis of PLM-E1 transfected ARVMs and confocal microscopy, we showed that the newly developed PLM-E1 biosensor is associated with the $\alpha_{1}$ subunit (SU) of the NKA. The obtained FRET results were compared with measurements in the bulk cytosol performed using the cytosolic E1-camps sensor. It is well known that PDE2 activity constitutes a relatively low proportion of total cardiac PDE activity in the rat heart. However, our findings suggest that the actions of PDE2 regulate CAMP signals in the PLM microdomain in a compartmentalized manner at basal state (without prestimulation with $\beta$-AR agonists). Using the targeted FRET biosensor PLM-E1 in ARVMs we further analyzed the subtype specific $\beta$-AR control of cAMP in the PLM/NKA domain. Interestingly, the $\beta_{2}$-AR showed a distinct control over the PLM microdomain during adrenergic stimulation. Local CAMP-FRET responses to PDE3 inhibition were well detectable in the PLM/NKA microdomain and showed a significant effect of this PDE in confining $\beta_{2}-A R$ signals to the 
vicinity of PLM upon adrenergic stimulation. Focusing on $\beta_{2}-A R$ stimulated cAMP pools in the PLM/NKA microdomain, we observed alterations of FRET responses in ARVMs from animals with chronic heart failure induced by myocardial infarction. These failing cells showed a significant loss of the PDE3 responses upon $\beta_{2}$-AR stimulation and an almost compensating increase in PDE2 dependent control of cAMP in the vicinity of PLM. Strikingly, in this disease model the overall response of $\beta_{2}$-AR to adrenergic stimulation in the PLM microdomain was reduced to a level comparable to the bulk cytosol.

In this study, the efficiency of the targeted PLM-E1 biosensor and its potential for real time monitoring of compartmentalized cAMP signaling in adult rat ventricular myocytes was successfully demonstrated. In addition, the practicability of the tagged biosensor in ARVMs in a cardiac disease model was confirmed and analyzed. In particular, our data show that real time dynamics of CAMP in the PLM/NKA microdomain are significantly different from cytosolic cAMP in terms of local PDE regulation and direct receptor-mediated control of the microdomain. In heart failure, these mechanisms are seriously altered which might explain impaired regulation of PLM in disease, bringing out a potential therapeutic target. 


\section{Introduction}

\section{1. $1 \beta$-adrenergic signaling pathway}

\subsection{Subtype specific $\beta$-adrenergic signaling in the heart}

As classic members of heptahelical G protein-coupled receptors (GPCRs), $\beta$-adrenergic receptors ( $\beta$-ARs) are important mediators of sympathetic nervous system control. They are functionally involved in various processes such as metabolic regulation ${ }^{1}$, immune reactions ${ }^{2}$, growth control ${ }^{3}$, vascular smooth muscle cell contraction and relaxation ${ }^{4}$ as well as cell survival and apoptosis 5 . The textbook paradigm for $\beta$-AR signaling in the heart is the regulation of cardiac function via their activation by catecholamines; the sympathetic stimulation increases heart rate (positive chronotropic effect), myocardial contractility (positive inotropic effect) and accelerates relaxation (positive lusitropic effect) $)^{6}$. There are three different types of $\beta$-ARs expressed in the mammalian heart: $\beta_{1^{-}}, \beta_{2^{-}}$and $\beta_{3^{-}}$ARs. The $\beta_{1}-A R$ is found primarily in the heart and comprises $75-80 \%$ of the cardiac $\beta$-ARs ${ }^{7}$. This receptor is coupled to stimulatory $G$-proteins $\left(G_{s}\right)$ which in turn activate adenyl cyclase $(A C)$, an enzyme responsible for the generation of the second messenger $3^{\prime}-55^{\prime}$-cyclic adenosine monophosphate (cAMP) from ATP. Increasing CAMP levels activate cAMP-dependent protein kinase (PKA) $)^{8}$, cyclic nucleotide gated channels (CNGCs) $)^{9}$ and the exchange protein directly activated by CAMP (Epac) ${ }^{10,11}$ (Figure 1). The latter seems to have prohypertrophic effects upon chronic $\beta_{1}-A R$ stimulation, being a PKA independent downstream effector of sympathetic stimulation. Epac expression is increased in heart failure, where Epac activates Rap (a small GTPase) and induces hypertrophy in a cAMP-dependent but PKA-independent manner ${ }^{12}$. In the heart, cAMP signal transduction is mainly mediated through PKA with its two regulatory $(\mathrm{R})$ - and two catalytic $(\mathrm{C})$-subunits ${ }^{8}$. When cardiac cAMP binds the R-subunits, the $\mathrm{C}$-subunits dissociate from the R-subunits ${ }^{13}$, which leads to a phosphorylation of downstream targets critical for the regulation of excitation contraction coupling (ECC). During cardiac ECC downstream effector proteins such as troponin I, the L-type $\mathrm{Ca}^{2+}$ channel (LTCC), phospholamban (PLB) ${ }^{14}$ and phospholemman (PLM) as the inhibitory protein of the sodium potassium pump (NKA) $)^{15}$ serve as PKA substrates, resulting in greater contractility and acute cardiac performance thereby facilitating diastolic function of the heart.

Contraction force is primarily controlled by sympathetic stimulation via $\beta_{1}-A R$ CAMP and elevated intracellular $\mathrm{Ca}^{2+}$ levels upon phosphorylation of voltage-gated LTCC, whereas the lusitropic effect of PKA is mediated by increased $\mathrm{Ca}^{2+}$ reuptake into the sarcoplasmic reticulum (SR) consequent to PLB phosphorylation and increased SERCA2a activity ${ }^{16}$. As an important part of the sympathetic 'fight or flight' response the NKA pump activity is upregulated by the PKA phosphorylation of PLM when sodium is pumped out of the cell in 
response to elevated intracellular $\mathrm{Na}^{+}$levels through the $\mathrm{Ca}^{2+}$ transient driven $\mathrm{Na}^{+}$entry via the $\mathrm{Na}^{+} / \mathrm{Ca}^{2+}$ exchanger $(\mathrm{NCX})^{17}$ (see section 1. 2. 1). Supported by several investigations about the effects of $\mathrm{Ca}^{2+} /$ calmodulin-dependent protein kinase II (CaMKII) upon $\beta$-AR stimulation, there is a great possibility that excessive $\beta-A R$ stimulation is detrimental because of CaMKII mediated hyperphosphorylation of $\mathrm{Ca}^{2+}$ handling proteins (e.g. ryanodine receptor, RyR2, phosphorylation leading to diastolic SR $\mathrm{Ca}^{2+}$ leak) ${ }^{18}$.

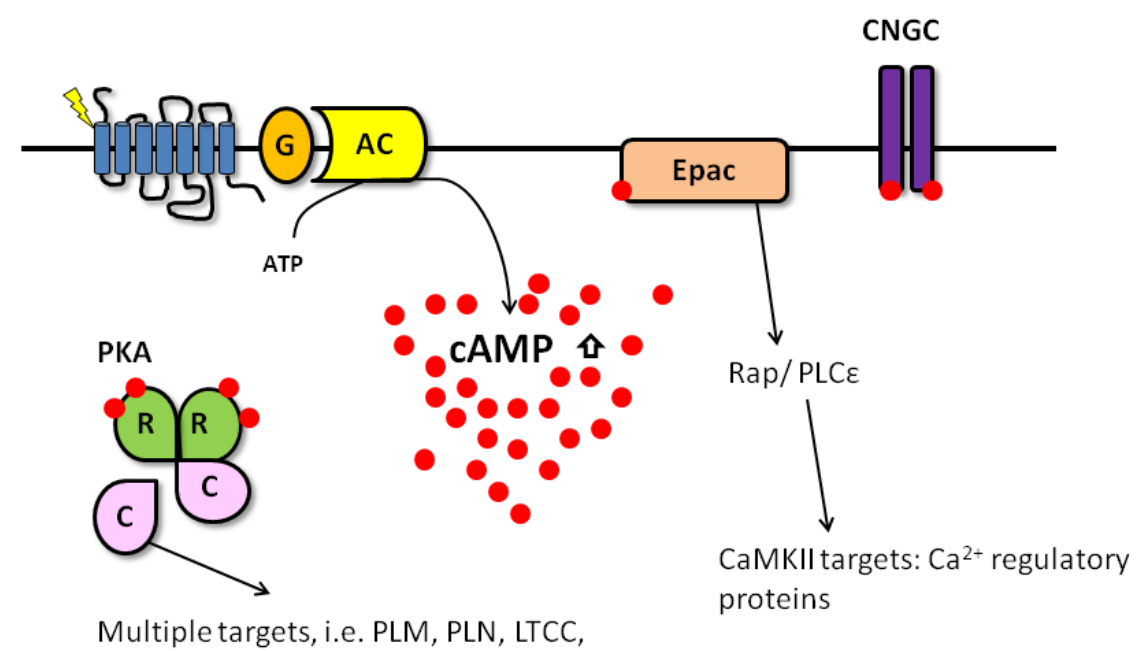

Troponin I, RyR, PDEs, nuclear CREB

Figure 1. Schematic representation of the cellular cAMP signaling cascade. After stimulation of a GPCR, cAMP production is accelerated via $G$ protein dependent $A C$ activation. CAMP has three different downstream effectors: PKA, Epac and CNGCs. The catalytic $(C)$ subunits of the PKA dissociate from the regulatory $(R)$ subunits and phosphorylate several downstream targets such as PLM, PLN, LTCC, Troponin I, RyR, PDEs and CREB. Epac and the small GTPases Rap, in response to CAMP, activate CaMKII through phospholipase $\mathrm{C} \varepsilon(\mathrm{PLC} \varepsilon)$ to regulate cardiac $\mathrm{Ca}^{2+}$ handling proteins. CNGCs show an increase in ion currents upon cAMP binding to these channels.

The $\beta_{2}$-AR comprises $20-25 \%$ of cardiac $\beta-\mathrm{ARs}^{7}$ and is expressed in several other tissues such as in the lung ${ }^{19}$ and kidney ${ }^{20}$. There is growing evidence for highly localized $\beta_{2}-A R$ dependent $A C / C A M P / P K A$ signaling ${ }^{21}$. Previous studies in cardiac myocytes ${ }^{22-24}$ have shown an inotropic effect of $\beta_{2}$-ARs mediated cAMP without having a direct influence on the phosphorylation status of PKA targets important for cardiac contraction, like PLB. It was suggested that $\beta_{2}-A R$ stimulation causes greater stimulation of the $G_{s}-A C$ system and has a greater efficacy compared to the $\beta_{1}-A R^{25-27}$. Not only for this reason, cardiac $\beta_{2}-A R$ mediated CAMP signal transduction has been described to be quantitatively and qualitatively different from $\beta_{1}-A R$ induced signals ${ }^{28}$ and markedly altered in heart failure ${ }^{29}$. However, in addition to $G_{s}$, the $\beta_{2}-A R$ couples to pertussis toxin - sensitive inhibitory $G$ protein $\left(G_{i}\right)^{22}$.

Cehn-Izu et al. suggested, that the local $\beta_{2}$-AR signaling character is $G_{i}$ dependent; the joint signaling of $\beta_{2}-A R$ to both $G_{s^{-}}$and $G_{i}$-proteins leads to a highly localized $\beta_{2}-A R$ signaling pathway to modulate sarcolemmal L-type $\mathrm{Ca}^{2+}$ channels in rat ventricular myocytes ${ }^{30}$. Through the additional signaling pathway, the $\beta_{2}-A R-t o-G_{i}$ branch might protect cells from 
apoptosis induced by sustained sympathetic $G_{s}$ mediated stimulation ${ }^{31}$. One theorized, that the switch of the $\beta_{2}-A R$ to $G_{i}$ coupled survival pathway via phosphoinositide 3-kinase (PI3K)/Protein Kinase B (Akt) may be caused by PKA phosphorylation of the receptor and serve as a protective mechanism against catecholamine cardiotoxicity ${ }^{32}$. In heart failure, $\beta_{1^{-}}$ ARs start to get desensitized via ligand stimulated phosphorylation by associated G proteincoupled receptor kinases (GRKs) ${ }^{33}$. GRKs prepare the activated receptor for internalization via the recruitment of $\beta$-arrestin $\left(\beta\right.$-Arr) ${ }^{34}$, and the receptor loses its functional quality in addition to their desensitization by GRKs in spite of the increased circulation of catecholamines $^{35-37}$. On the other hand, $\beta_{2}$-ARs may support the failing/aging heart by enhancing myocardial contractility ${ }^{38,39}$ (Figure 2).

The $\beta_{3}-A R$ is found primarily in adipose tissue where it is coupled to $G_{s}$-proteins ${ }^{40}$, and little in the heart. Previous studies in $\beta_{1} / \beta_{2} A R-K O$ mice have suggested, that the Isoprenaline (nonselective $\beta$-AR agonist, Iso) induced $\beta_{3}$-AR signaling leads to very modest cardiac contractility and seems to desensitize more rapidly than does signaling by either the $\beta_{1} A R$ or the $\beta_{2} A R^{41}$. Conversely, the cardiac-restricted overexpression of $\beta_{3}-A R$ in a transgenic mouse model showed enhanced cardiac contractility after treatment with a selective $\beta_{3}-A R$ agonist ${ }^{42}$. Reasons for disparities in $\beta_{3}$-AR effects may be attributed to the use of different compounds and different transgenic animal models. It has been reported that $\beta_{3} A R$ couples to $G_{s}$ - and $\mathrm{G}_{\mathrm{i}}$-proteins in diverse cell types ${ }^{43,44}$. Recently, it was also demonstrated that cardiomyocyte expression of this receptor is increased in rodent and human failing heart and it might be cardioprotective by its coupling to $G_{i}$ and nitric oxide synthase-dependent NO/soluble guanylyl cyclase/cGMP pathway ${ }^{45,46}$.

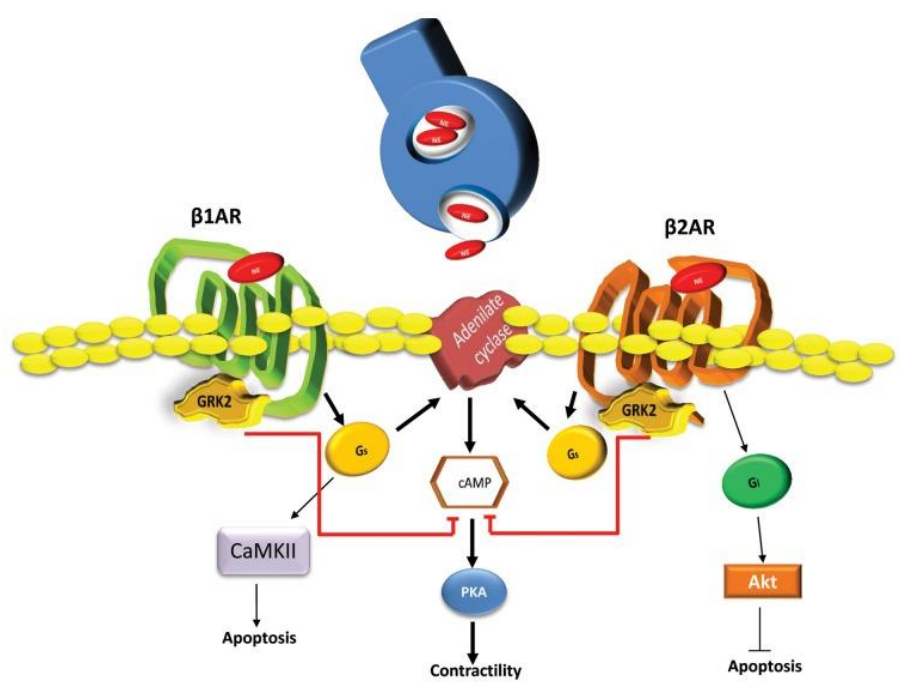

Figure 2. Cartoon describing $\beta_{1}$-ARs and $\beta_{2} A R s$ signaling pathways in cardiomyocytes. $G_{s}$ coupled $\beta_{1}$-ARs activates PKA through the production of cAMP resulting in positive chronotropic and inotropic effects. Secondly, activated CaMKII can induce apoptotis with increased catecholamine stimulation. In contrast, antiapoptotic signaling through Akt is mediated by $G_{i}$ dependent $\beta_{2}$-ARs. Both $\beta$-ARs are regulated by GRK2 leading to receptor downregulation in case of chronic activation. Taken from Cannavo et al. ${ }^{29}$. 


\section{1. 2 CAMP - one second messenger with several functions}

Discovered in 1958 by Earl W. Sutherland and co-workers ${ }^{47}$ cAMP remains to be interesting for many researchers as a universal second messenger that produces different physiological effects depending on cell nature and composition. cAMP plays central roles in memory formation $^{48}$ and mid brain system ${ }^{49}$, the secretion of insulin from pancreatic cells ${ }^{50,51}$, regulation of gene expression ${ }^{52}$ and metabolism ${ }^{53}$, immune reactions ${ }^{54}$ and most important for this work: cardiovascular regulation ${ }^{55}$. These effects can even be opposing in different cell types. For example, cAMP increases cardiac cell contractility but relaxes airway and vascular smooth muscle cells ${ }^{56}$. Moreover, studies on rat ventricular myocytes provided first insights into the idea of the spatially segregated cAMP signals organized in microdomains containing several proteins as molecular components of the signaling pathway (see section 1. 1. 3). Briefly, cardiomyocytes stimulated by the $\beta$-adrenergic agonist isoprenaline (Iso) or by prostaglandine $E_{1}$ led to comparable amounts of synthesized cAMP resulting in PKA activation in both cases, but only Iso treated cells augmented cardiomyocyte contractility. The conclusion was that prostaglandine $E_{1}$ mediated cAMP showed no classical substrate phosphorylation (especially at cardiac troponin I) due to the activation of different pools of PKA within the cardiomyocyte ${ }^{57}$. Likewise, cAMP signals elicited from glucagon receptors were demonstrated as not being involved in cardiac contractility in contrast to cAMP generated through $\beta$-ARs after Iso stimulation, which was another example of functionally distinct AC/cAMP/PKA microdomain ${ }^{58}$. The idea that not all cAMP pools and its respective downstream effects in the heart are equal, was established and demonstrated in several other studies on cardiac myocytes ${ }^{23,59,60}$. As mentioned above, there are differentially localized PKA subsets of PKA type I and PKA type ${ }{ }^{61}$, defined by their varying subunit isoforms and by differential binding to a variety of A-kinase anchoring proteins (AKAPS). This anchor proteins can also bind phosphodiesterases (PDEs), enzymes responsible for cAMP degradation, ensuring localized complexes and specific physiological responses to any given CAMP stimulus ${ }^{62}$. Next to the idea that CAMP has physical membrane barriers formed by different kind of organelles inside the cell ${ }^{63,64}$, PDEs have a key role in shaping intracellular gradients of CAMP and disabling random cAMP diffusion ${ }^{65}$.

Most studies were focused on comparing CAMP dynamics between soluble and particulate fractions $^{66,67}$, but it was also clear that the $\beta$-AR-AC-cAMP-PKA signaling pathway is not uniformly active at the plasma membrane either. For example, there are differences in lipid raft (cholesterin and sphingolipid rich planar domains) and non-lipid raft membrane domains $^{10,68}$. Caveolae (omega shaped membrane invaginations) represent a special type of cardiac lipid rafts, containing caveolin (Cav) proteins as main components. These membrane domains have been shown to harbor $\beta$-AR-cAMP pathway components important for $\mathrm{Ca}^{2+}$ cycling ${ }^{69,70}$ forming, for example, a macromolecular complex comprised of receptor and L- 
type $\mathrm{Ca}^{2+}$ channel (LTCC) in the heart ${ }^{71}$. Furthermore, studies demonstrated that such gradients of cAMP specifically derived from $\beta_{2}-\mathrm{ARs}^{29}$ activate a subset of protein kinase $A$ molecules anchored in proximity to T-tubules ${ }^{72}$ (large sarcolemmal invaginations at $Z$-lines of cardiomyocytes). Cardiac LTCCs are proposed to be a sarcolemmal substrates of PKA ${ }^{73,74}$ and presumably organized in cardiac T-tubules together with NKA and NCX ${ }^{75-78}$. There is an increasing body of evidence that PLM is a central player in the sympathetic response by kinase dependent inhibition of the NKA activity and indirect regulation of the cellular calcium load and inotropy of the heart ${ }^{15}$. In this context, elements important for the local tailoring of cAMP concentrations to distinct compartments and protection of possible substrates against inappropriate PKA phosphorylation within a cell are pointed out in the next section.

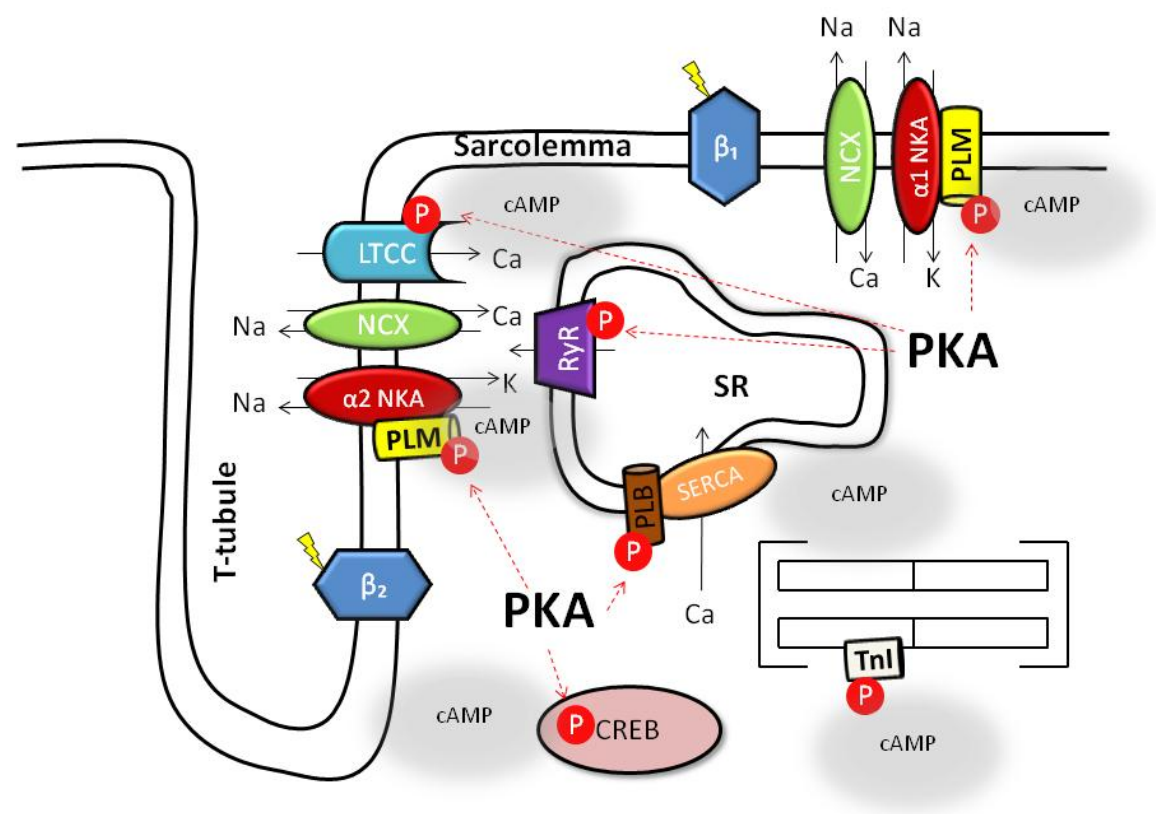

Figure 3. Cartoon depicting the differential role of cardiac cAMP in confined microdomains leading to differential phosphorylation of PKA targets. During the action potential, $\mathrm{Ca}^{2+}$ flows into the cell, which triggers $\mathrm{Ca}^{2+}$ dependent $\mathrm{Ca}^{2+}$ release from the SR facilitating $\mathrm{Ca}^{2+}$ binding to troponin I (Tnl) at myofilaments and contraction ${ }^{151}$. The NKA is found in both sarcolemma and T-tubules regulating cellular $\mathrm{Ca}^{2+}$ and $\mathrm{Na}^{+}$ concentrations functionally linked to the NCX, which predominantly removes intracellualar $\mathrm{Ca}^{2+}$ (forward mode $)^{172}$ during diastole. Upon $\beta$-AR stimulation, cAMP activates PKA, which phosphorylates several downstream targets important for the cardiac excitation contraction coupling such as LTCC, RyR2, PLN, PLM and Tnl, thereby increasing chronotropy, inotropy and lusitropy. The different $\mathrm{Ca}^{2+}$ and $\mathrm{Na}^{+}$handling proteins are part of cAMP microdomains containing additional pools of PKA and PDEs (not shown here for simplicity).

\section{1. 3 cAMP compartmentation in the heart - role of Phosphodiesterases and AKAPs}

The compartmention of cAMP derived signals in functional microdomains and their specific effects are widely recognized and demonstrated in different kinds of cells over the years. Most remarkable attempt showing local PDE activity in distinct intracellular microdomains was by whole cell patch clamp recordings which indicated a local PDE dependant cAMP pool 
involved in $\beta$-AR stimulation of LTCC currents in frog ventricular myocytes ${ }^{70}$. Aberrant cAMP signals and changes in highly regulated microdomains may occur in diseased cells and contribute hypertrophy and heart failure ${ }^{79}$. For this reason it is of extreme importance to maintain the architecture of positively and negatively CAMP modulating and scaffolding proteins in the given microdomains.

PDEs as CAMP degrading enzymes catalyze its hydrolysis and are critical for shaping the CAMP pools in mammalian cells ${ }^{65,80}$. This enzyme superfamily is comprised of 11 families and 70 different isoenzymes, which are encoded by 21 genes ${ }^{81}$. Cardiac myocytes express several PDE families: PDEs 1-5, 8 and $9^{82,83}$. PDEs are categorized in their affinity and catalytic rates, in which PDEs 1-3 can hydrolyze both cAMP and 3',5'-cyclic guanosine monophosphate (cGMP), while PDE4 and 8 are cAMP specific and PDE5 and 9 show a high selectivity for cGMP. Besides their kinetics, various PDE enzymes have differences in regulatory properties and sensitivity to inhibitors ${ }^{84}$ (see Table 1).

Table 1. Cardiac PDE enzyme specifications and kinetic properties; $\mathrm{Km}=$ Michaelis-Menten constant, Vmax $=$ maximal velocity (maximal reaction rate), - no information available; modified from Eiki Takimoto ${ }^{73}$

\begin{tabular}{|c|c|c|c|}
\hline \multirow{2}{*}{ PDE Isoenzyme } & \multicolumn{2}{c|}{ Km $(\mu \mathrm{M})$} \\
\cline { 2 - 4 } & $\mathrm{Ca}^{2+} /$ CaM regulated, dual specificity & PDE1A: $73-120$ & PDE1 A: $2.6-5$ \\
& & $\begin{array}{c}\text { PDE1B: } 10-24 \\
\text { PDE1C: } 0.3-1.2\end{array}$ & $\begin{array}{c}\text { PDE1B: } 1.2-5.9 \\
\text { PDE1C: } 0.6-2.2\end{array}$ \\
\hline PDE1 & $30-50$ & $10-30$ \\
\hline PDE2 & cGMP stimulated, dual specifity & $0.02-0.15$ & 0.18 \\
\hline PDE4 & cGMP inhibited, dual specifity & $2.9-10$ & - \\
\hline PDE5 & cAMP-specific & - & $1.0-6.2$ \\
\hline PDE8 & cGMP-specific & 0.15 & - \\
\hline PDE9 & cAMP-specific & - & $0.17-0.39$ \\
\hline
\end{tabular}

PDE inhibitors are commonly used to characterize subcellular contributions of PDEs to cAMP hydrolysis and spatio-temporal dynamics ${ }^{72,85}$, dissecting their key roles in shaping intracellular microdomains (see section 1.4).

In particular, PDE1 has three subfamilies (PDE1A, PDE1B and PDE1C). PDE1 enzyme activity is increased via $\mathrm{Ca} 2+/$ calmodulin binding at the regulatory domain in the $\mathrm{N}$-terminus, PDE1 is also often referred to as the $\mathrm{Ca}^{2+} /$ calmudilin stimulated $\mathrm{PDE}^{86}$. Having different affinities for both cyclic nucleotides (dual substrate specificity), PDE1 isoenzymes are believed to be important in the crosstalk between the second messenger $\mathrm{Ca}^{2+}$ and cyclic nucleotide signaling ${ }^{87}$. Albeit PDE1 has been suggested to play a role in cardiac disease ${ }^{88,89}$, 
there is still a need for potent selective PDE1 inhibitors to better investigate a possible cardioprotective effect.

PDE2 is another dual specific enzyme family with three cardiac isoforms (PDE2A1-3). cGMP can enhance the catalytic activity of PDE2 through binding to a special regulatory domains at the N-terminus, referred to as GAF domains ${ }^{90}$ (found in: cGMP-specific phosphodiesterases, Adenylyl cyclases and FhIA). Via an allosteric binding mechanism, this leads to a direct increase of cAMP hydrolysis (positive cGMP/CAMP crosstalk). Mongillo and co-workers have found that PDE2 is strongly involved in cGMP/CAMP interplay after $\beta$-adrenergic stimulation $^{91}$. Using real time Fluorescence Resonance Energy Transfer (FRET)-based imaging in neonatal rat cardiomyocytes, they showed that 'cGMP activated' PDE2 modulation of cAMP was potentiated upon cGMP production via the $\beta_{3}-A R / N O S / c G M P$ pathway which exhibited a negative inotropic effect after beta-AR stimulation in neonatal rat cardiomyocytes $^{91}$. Furthermore, studies in ventricular myocytes of guinea pigs ${ }^{92}$ and ARVMs $^{93}$ have supported their findings that PDE2 is highly membrane associated.

In contrast to PDE2, cGMP inhibits the hydrolyzing activity of PDE3 for both cyclic nucleotides. This competitive inhibition provides a means by which an increase in cGMP may lead to an increase in CAMP and its differential effects in spatially distinct subcellular domains (negative cGMP/cAMP crosstalk) ${ }^{94}$. PDE3 comprises two subfamilies PDE3A and PDE3B. Although both are present in cardiomyocytes, PDE3A is the main subfamiliy ${ }^{95}$ responsible for cardiac hypertrophy ${ }^{96}$ and contractility which is typically augmented by PDE3 inhibition ${ }^{97}$. PDE3 are described to be in the cytosolic fractions as well as membrane bound isoenzymes, depending on the isoform PDE3A1 (membrane and soluble), A2 or A3 (both are only membrane bound) ${ }^{98,99}$. However, PDE3A is found mainly at the SR/endoplasmic reticulum rather than at the plasma membrane ${ }^{100}$. PDE3 is a dual specific enzyme and displays high affinity for both CAMP and CGMP, having higher catalytic activity for CAMP than for CGMP $^{101}$. A decrease in PDE3 expression is thought to have adverse effects on the heart as it promotes cardiomyocyte apoptosis and impairs cardiac dysfunction induced by chronic pressure overload $^{102}$. PDE3 and PDE4 contribute the majority of the cAMP catabolism in cardiomyocytes $^{65}$.

PDE4, the CAMP specific PDE, is the most diverse PDE family with four encoding genes (PDE4A, B, C and D) and around 20 splice variants ${ }^{103}$. In the mammalian heart, three out of four genes (PDE4A, B and D) encode for PDE4 isoenzymes ${ }^{102,104}$. The N-terminal regulatory domain determines the subcellular localization of the PDE4 isoforms ${ }^{105}$ and at the same time makes them essential players in cAMP compartmentation in cardiomyocytes. With the $\mathrm{N}$ terminal regulatory/localization domain PDE4 variants are able to form local cAMP signaling hubs by binding to scaffolding proteins such as $\beta$-Arr or AKAPs and through direct interaction with $\beta$-AR receptors. For example, a fraction of PDE4B is colocalized with the LTCC along T- 
tubules in the mouse heart, thereby controlling the $\beta$-AR stimulation of the L-type $\mathrm{Ca}^{2+}$ current and the phosphorylation of the LTCC via PKA ${ }^{106}$. There is evidence that the PDE4D3 isoform and the PKA reside in the same signaling complex through their association with the muscle-selective A-kinase anchoring protein (mAKAP), which in turn enhances the PDE activity, forming a unique negative feedback control over rising of local CAMP in cardiomyocytes $^{107}$. Moreover, PDE4D3 was found in the cardiac RyR2/calcium-releasechannel complex ${ }^{108}$. Playing a key role in cellular desensitization mechanism for GPCRs (see section 1.1.1), PDE4D5 has been demonstrated to recruit $\beta$-Arr to the $\beta_{2}$-AR upon receptor stimulation $^{109,110}$. The PDE4D8 isoform was reported to form signaling complexes with $\beta_{1}$ ARs until binding of an agonist ${ }^{110}$. Phosphoinositide 3-kinase $\gamma(\mathrm{PI} 3 \mathrm{~K} \gamma)$ is another newly identified regulator of PDE action. It controls local $\beta_{2}$-AR-CAMP responses by activation of the cardiac PDE4A, PDE4B and PDE3A isoforms through PKA. By doing this $\mathrm{PI} 3 \mathrm{~K} y$ recruits the regulatory subunit of PKA to and the PDE isoforms, respectively, to the functional signaling complex. The absence of $\mathrm{PI} 3 \mathrm{~K} y$ showed hyperphosphorylation of the L-type calcium channel $\left(\mathrm{Ca}_{v} 1.2\right)$ and phospholamban which resulted in proarrythmic changes ${ }^{111}$.

PDE5 is one of the most intensively studied enzymes for cGMP hydrolysis. PDE5 inhibitors show some benefits in hypertrophy and heart failure ${ }^{112,113}$. There are clinically used PDE5 inhibitors officially approved for treatment of erectile dysfunction (PDE5A resides in the corpus cavernosum ${ }^{114}$ ) and pulmonary hypertension ${ }^{115}$. The three known isoforms PDE5A1-3 also contain a regulatory GAF domain with binding sites for cGMP like PDE2 does, but this leads to a stronger increase in cGMP catalytic activity in contrast to PDE2 ${ }^{84}$. One other difference between both cGMP hydrolyzing PDEs is the subcellular distribution (cGMP compartmentation); Castro et al. demonstrated that PDE5 controls cGMP originating from the particulate pool of guanylyl cyclase (GC), whereas PDE2 controls cGMP in the soluble fraction $^{93}$.

PDE8 is another cAMP-specific PDE in the mammalian myocardium, which is encoded by two genes (PDE8A and PDE8B). PDE8A is highly expressed in testis and ovary of mouse and human cells ${ }^{116,117}$, whereas PDE8B is found to be expressed specifically in thyroid glands ${ }^{118}$ in humans. PDE8 is distinguished from other cAMP specific proteins in the heart (PDE4) by its insensitivity to most of the PDE inhibitors including Isobutyl-1-methylxanthin (IBMX), however it can be inhibited by dipyridimole ${ }^{116}$. In the heart, PDE8A can be phosphorylated by the PKA holoenzyme ${ }^{119}$, similar to PDE4. Additionally, the same isoenzyme plays a regulatory role in the calcium transients during the $\mathrm{ECC}^{120}$.

The fifth PDE family PDE9 contain a cardiac PDE9A which is a high affinity cGMP-specific phosphodiesterase expressed at the mRNA level in healthy human but not in mouse heart ${ }^{121}$. This new phosphodiesterase is similar to PDE8A regarding its insensitivity to most of the commonly used inhibitors apart from zaprinast, a PDE5 inhibitor. Lee et al. demonstrated in 
recent studies that unlike PDE5A, PDE9A can regulate cardiac cGMP signaling independent of the nitric oxide pathway and selective targeting of PDE9A could protect against sustained pressure-overload stress in cardiac diseases ${ }^{122}$.

A-Kinase Anchoring Proteins (AKAPs) are highly important in cardiac cAMP signaling amplitude and duration. Several cardiac AKAPs have been described as functionally essential scaffolding proteins, which selectively localize cAMP mediated PKA signaling to subcellular microdomains ${ }^{123-126}$ (Figure 4). At least 15 AKAPs were identified in cardiac cells, most of which are involved in recruiting PKA isoforms, PDEs and protein phosphatases (PPs) in order to confine relevant signaling complexes in the heart ${ }^{127}$. AKAP18a is found in association with the LTCC to facilitate the PKA-dependent phosphorylation of the channel at the sarcolemma ${ }^{73}$. AKAP79/150 (in human/murine cardiomyocytes) assembles large signaling complexes with PKA, calcineurin, Cav-3 and AC at the T-tubular area, thereby facilitating PKA phosphorylation of LTCC, RyR2; and even PLB phosphorylation is AKAP150 dependent at its Cav-3 associated domains ${ }^{128,129}$. Confinement of $\beta$-AR/cAMP signals, phosphorylation and dephosphorylation of PLB at the SERCA2 compartment is achieved through AKAP18 $\delta^{130,131}$. Additionally, PKA anchoring via AKAP9 (Yotiao) to the $\mathrm{K}^{+}$channel is highly important in terms of rapid cardiac repolarization of cardiomyocytes ${ }^{132}$. PKA phosphorylation of the potassium channel is regulated by PDE4D3, also recruited by Yatiao, navigating CAMP effects on the $\mathrm{K}^{+}$channel activity ${ }^{133}$. As mentioned above, muscle-selective mAKAP mediate the dynamics of PKA activation within the RyR2 complex tightly regulated ${ }^{134}$ by PDE4D3 and by AC5 activities ${ }^{135}$, since both enzymes can directly bind to this anchoring protein. In this context, it has been demonstrated that mAKAP mediates PKA-induced phosphorylation of the RyR2 as a scaffold protein (Figure 4).

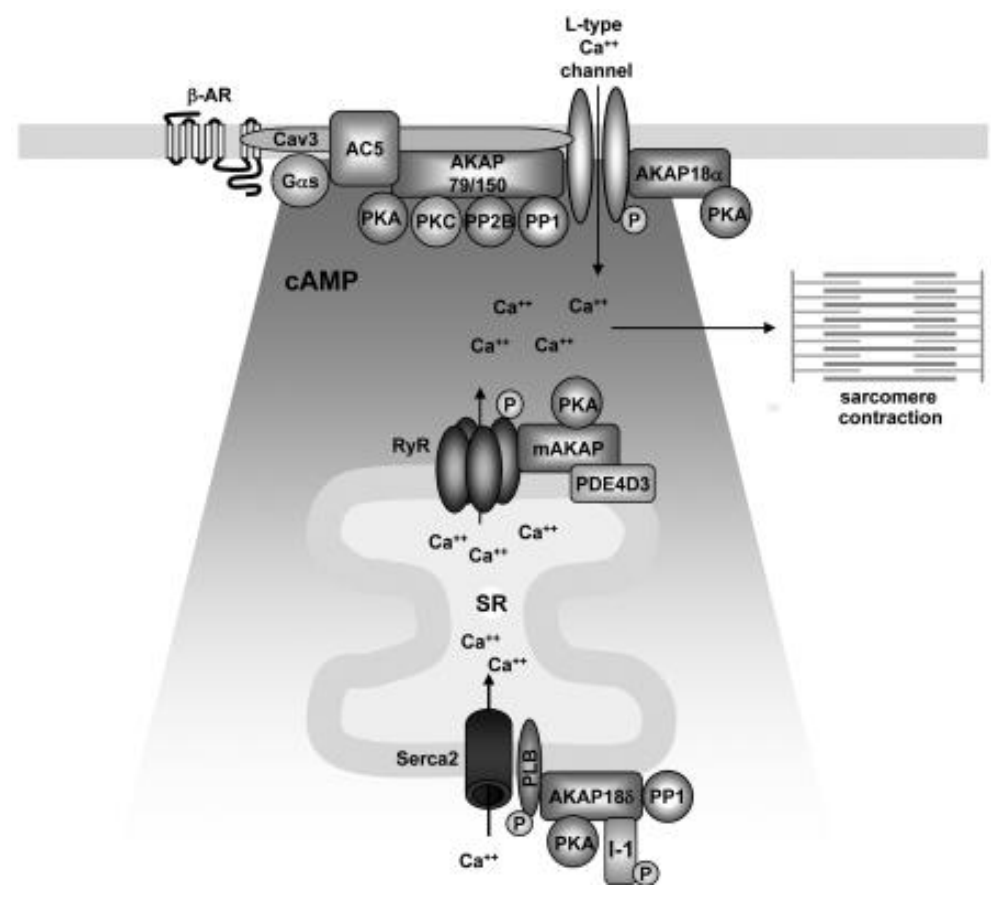

Figure 4. A-kinase anchoring proteins (AKAPs) controlling subcellular CAMP microdomains in the heart. Important AKAPs contributing to CAMP compartmentation and targeted PKA phosphorylation of key-regulatory proteins of the cardiac ECC. Taken from Diviani et al. ${ }^{108}$. 


\section{1. 4 Changes of local CAMP signaling and $\beta$-AR subtype modules in cardiac hypertrophy}

Rat model of congestive heart failure (CHF) syndrome after myocardial infarction (MI) is the most commonly used experimental model to study the alteration and progression of cardiac physiology during progression to heart failure ${ }^{136}$. By ligation of the left anterior descending artery, a method usually used for creating cardiac ischemia ${ }^{137}$, irreversible myocardial injury and loss of cardiac myocyte function is observed in the infarct area. With ventricular remodeling and resulting hypertrophied cardiac muscle, the heart compensates for the diminished oxygen supply without losing much of its contractility ${ }^{138}$ (compensated hypertrophy). This compensatory response is limited by the degree of infarct size; for example, if very large amounts of myocardium become necrotic, there is not enough hypertrophy to normalize myocardial volume. To sustain normal perfusion pressure, the renin-angiotensin-aldosterone system is activated promoting ventricular remodeling, progressive dilatation and deterioration in myocardial function ${ }^{139,140}$ (decompensated hypertrophy). The sympathetic activation of $\beta$-AR receptors is a critical component of the progression of heart failure, being a 'good' response under stress ("fight-or-flight" response) or for acute myocardial infarction to maintain cardiac performance, but is recognized as detrimental in a long-term view ${ }^{141,142}$. As mentioned before, in response to accelerated release of catecholamines in progressive heart failure, there is desensitization and reduction of $\beta$-AR density in the myocardium ${ }^{37,143}$.

Studies have shown that remodeling during the development of heart failure after MI starts at the cell membrane level, where the T-tubular network is altered ${ }^{144,145}$ leading to impairment of CAMP microdomains and subtype-specific $\beta$-AR signaling during heart failure. By combining scanning ion conductance microscopy (SICM) with the FRET based imaging technique, Nikolaev et al. have demonstrated a redistribution of $\beta_{2}-A R$ and normally highly confined $\beta_{2^{-}}$ AR mediated CAMP signals from T-tubules to detubulated membrane areas ${ }^{29,146}$. Impaired $\beta_{2}$-AR regulation via PI3K $y$ controlled PDE3-dependent cAMP pools close to the plasma membrane was demonstrated to be a potential cause of ventricular arrhythmias which are often observed in heart failure ${ }^{111}$.

During cardiac disease alterations in cAMP compartmentation and scaffolding by AKAPs and PDEs are observed. Several groups have shown that the PKA association with AKAPs is decreased in heart failure, for example due the pathological decrease in PKA-R subunit autophosphorylation ${ }^{147}$, which in turn decreases cardiac troponin I phosphorylation in the failing human and animal heart ${ }^{148,149}$. Disruption of the macromolecular complex of mAKAP/RyR/FKBP2.6/PDE4D3 leads to increased sensitivity to $\mathrm{Ca}^{2+}$ in failing human hearts. It has been demonstrated, that this can be either caused by distinct dissociation of FKBP2.6. ${ }^{150}$ (calstabin) from this complex due to RyR2 hyperphosphorylation ${ }^{151}$ or by 
maladaptive changes of the cAMP sensitive PDE4D3 localization due to pathophysiological changes of the heart ${ }^{108}$; both resulting in a 'leaky' RyR and consequent arrhythmogenesis by disturbed $\mathrm{Ca}^{2+}$ handling ${ }^{152}$. Interestingly, it has been suggested that mAKAP/RyR2 participates in early cardiac myocyte hypertrophy by adrenergic receptor signaling ${ }^{153}$.

Evidence provided by several groups have proven that different stages of impaired cardiac performance, from early disease stage (compensated hypertrophy) to the stage of chronic heart failure, are reflected by changes of PDE microdomain action in terms of expression and localization. Using a transgenic mouse model of caveolin targeted FRET biosensor Perera et al. uncovered cGMP-sensitive PDE2 and PDE3 redistribution between $\beta_{1}-A R-$ and $\beta_{2}-A R-$ associated membrane microdomains with unaltered whole-cell PDE expression and activities in early cardiac disease (Figure $5 \mathrm{~B}$ ). Recent experiments by Sprenger et al., demonstrated local increase in CAMP dynamics at the PLB/SERCA2a due to PDE3 and especially PDE4 down-regulation in order to compensate for the loss of SERCA2a function in the early disease mouse model. Furthermore it was indicated that the decreased PDE4 effects are replaced by local PDE2 responses during sympathetic stimulation, which suggest protection of the heart and especially the SERCA2a compartment against elevated cAMP levels in hypertrophy ${ }^{154}$.

Consistent with this notion, Mehel et al. showed that there is a significant PDE2 expression in human failing hearts, which constrains the effects of long-term $\beta$-AR stimulation on cardiomyocyte $\mathrm{Ca}^{2+}$ handling and contractility in $\mathrm{HF}^{155}$.

By contrast, the expression and activity of other cAMP-hydrolyzing enzymes, PDE3 and PDE4, are significantly down-regulated ${ }^{102,156}$. Under HF conditions, both the lower PDE3 levels promoting enhanced cardiomyocyte apoptosis and higher compensating PDE2 activity in late stage HF (Figure 5C) could be a consequence of increased cGMP, which is produced by guanylyl cyclase (GC) stimulated with increased levels of natriuretic peptides in hypertrophy.

PDE3A is suggested to be down-regulated in $\mathrm{HF}^{157}$ which results in induction of a proapoptotic transcriptional repressor gene ${ }^{156}$ in the PDE3 regulated cAMP-dependent nuclear microdomain. Treatment with PDE3 inhibitors (especially in congestive heart failure ${ }^{158}$ ) improves cardiac contractility but increases mortality rate due to arrhythmias and sudden cardiac death ${ }^{159}$.

Despite these detailed insights about alterations in global and local PDE effects (Figure 5) ${ }^{160}$, there is little known about changes of individual subtype-specific $\beta$-AR dependent PDE regulation of cAMP signaling to the vicinity of the cardiac PLM-NKA complex. This topic amongst others is addressed in this project. 


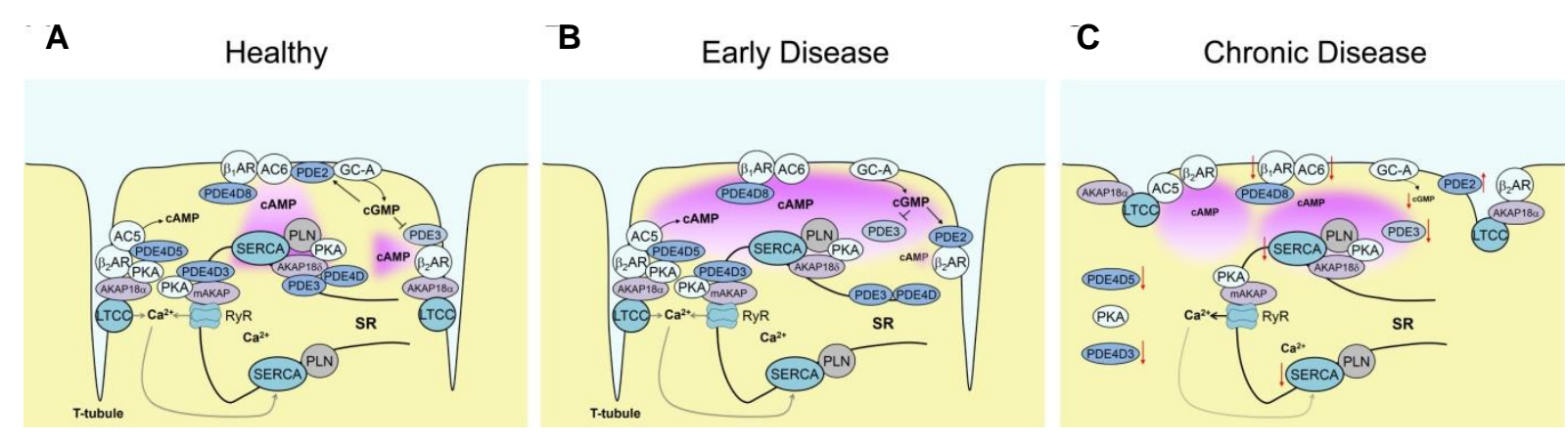

Figure 5. Alterations of local cAMP signaling in compensated hypertrophy and heart failure. (A) In healthy cardiomyocytes, cAMP is acting at major $\mathrm{Ca}^{2+}$ handling proteins, forming microdomains which contain local pools of PKA anchored to AKAPs and specific PDE isoenzymes. $\beta_{2}$-AR signals are predominantly located around LTCC in the T-tubules, whereas $\beta_{1}-A R$ signals originate from the surface membrane producing far-reaching cAMP acting in subcellular microdomains (e.g. to SERCA2a) by means of scaffolding proteins. (B) In compensated hypertrophy (early stage of HF), the total PDE activities are unaltered, albeit local decreases at cAMP compartments (PDE3 at $\beta_{2}-A R$ and PDE3/PDE4 at SERCA) and specific relocations occur (e.g. PDE2 between $\beta_{1}$ - and $\beta_{2}-A R$ ). (C) In heart failure (chronic cardiac disease), structural deformation of T-tubules and alterations in global and local PDE/PKA effects next to down-regulation and/or desensitization of $\beta_{1}$-AR take place. There is a decrease in receptor-microdomain coupling and functional cAMP responses. Taken from Froese et al. ${ }^{145}$.

\section{2 Differential regulation of the Na/K-ATPase by Phospholemman in the microdomain}

\section{2. 1 The cardiac sodium pump}

Discovered in 1957 by J.C. Skou the sarcolemmal sodium-potassium ATPase (NKA) ${ }^{161}$ is the predominant efflux source for cardiac sodium which maintains the transarcolemmal sodium gradient and provides a rapid upstroke of action potential ${ }^{162}$. This gradient ensures proper ion homeostasis, critical for sodium dependent membrane transporters (for example, $\mathrm{Na}^{+} /$glucose cotransporter) and for normal cellular function ${ }^{163}$. The action of the sodium pump regulates physiological electrical activity influencing the sodium-calcium exchanger (NCX) and cardiac contractility ${ }^{164}$ (Figure 6). The NKA extrudes three sodium ions from the cytosol to the extracellular domain in exchange for two potassium ions particularly to restore the steady-state intracellular sodium concentration at 4-8 $\mathrm{mM}(10-15 \mathrm{mM}$ in rat and

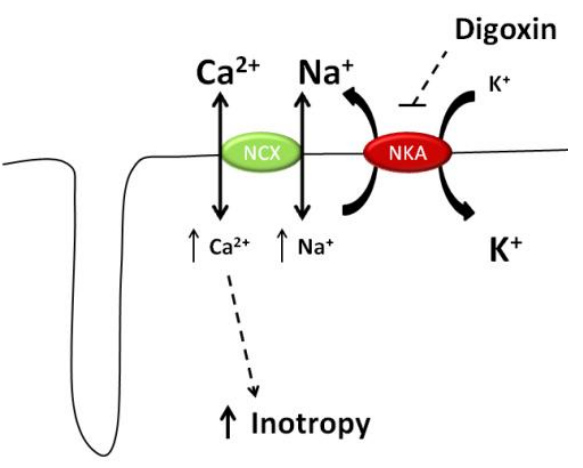

Figure 6. NKA - an integral player in the 'fight-or-flight' response. Enhancement of $\mathrm{Na} / \mathrm{K}$-ATPase activity limits the rise in intracellular $\mathrm{Na}^{+}$caused by the higher level of $\mathrm{Na}^{+}$influx during action potential. By doing so, NKA limits the rise in cellular and $\mathrm{SR} \mathrm{Ca}^{2+}$ load by favoring $\mathrm{Ca}^{2+}$ extrusion via the $\mathrm{Na} / \mathrm{Ca}$ exchanger. Inhibiting the NKA via cardiotonic steroids induces cardiac inotropy. mouse $)^{165}$ at the expense of metabolic energy driven from the hydrolysis of ATP ${ }^{166}$. This ion exchange results in one net charge generating a membrane current. For the quantification of sodium pump mediated (i.e., ouabain-suppressible) transport the $\mathrm{K}^{+}$analogue ${ }^{86} \mathrm{Rb}$ (Rubidium) is often used as a tracer for $\mathrm{K}^{+}$and gives reliable results ${ }^{167}$. 
Upon action potential $\mathrm{Na}^{+}$and $\mathrm{Ca}^{2+}$ enter the cell through voltage gated ion channels, which triggers $\mathrm{Ca}^{2+}$ from the sarcoplasmic reticulum (SR). Elevated $\mathrm{Ca}^{2+}$ triggers cellular contraction in the systole, which is followed by diastolic $\mathrm{Ca}^{2+}$ extrusion mainly via the NCX in forward mode which in turn is promoted by the NKA pumping $\mathrm{Na}^{+}$out of the cell to keep the $\mathrm{Na}^{+}$ levels constant ${ }^{164}$. Hence, cellular $\mathrm{Ca}^{2+}$ levels, that are highly important for the regulation of the ECC, are linked to the NKA activity. Elevated intracellular sodium would favor reverse mode of NCX resulting in larger calcium transient and contractility ${ }^{162}$. Since the 18th century this mechanism is made use for cardiotonic steroids. For example, digoxin as NKA inhibitor, induces acute cardiac inotropy and is used for treatment of heart failure ${ }^{168}$. Several groups have shown that a small transmembrane protein called phospholemman (PLM) interacts with the NKA and modulates it activity, analogous to phospholamban (PLB) at SERCA ${ }^{169}$, another P-type pump ${ }^{170-174}$. Upon $\beta$ - adrenergic stimulation in the heart, PLM works against cardiomyocyte sodium and calcium overload primarily by disinhibiting the NKA (discussed below).

\section{2. 2 Cardiac subunit expression and composition of the Na pump}

Solving of the crystal structure of the NKA in $2007^{175-177}$ revealed new insights about structure and composition of the cardiac sodium pump. In mammalians, four $\alpha\left(\alpha_{1}, \alpha_{2}, \alpha_{3}, \alpha_{4}\right)$ and three $\beta$ $\left(\beta_{1}, \beta_{2}, \beta_{3}\right)$ isoforms are recognized as minimum operative subunits (SUs) $)^{178,179}$. Having binding sites for $\mathrm{Na}^{+}, \mathrm{K}^{+}$, ATP and cardiac glycosides the catalytic $\alpha-S U$ assembles with the $\beta$-SU, which is highly important for structural and functional maturation of the sodium pump ${ }^{180}$, and one of the seven isoforms of the so called 'Fixid' proteins (FXYD), titled according to their conserved phenylalanine-X-tyrosine-aspartate motif $^{181,182}$. This functional multi-subunit enzyme (Figure 7) differs in tissue specific composition, proving that the sodium pump plays a key role in multiple regulatory mechanisms involving critical $\mathrm{Na}^{+}$ transport under different physiological conditions. Predominant isoforms in the heart are $\alpha_{1}, \alpha_{2}$ and $\beta_{1}$ having FYXD1 (PLM) as regulatory protein

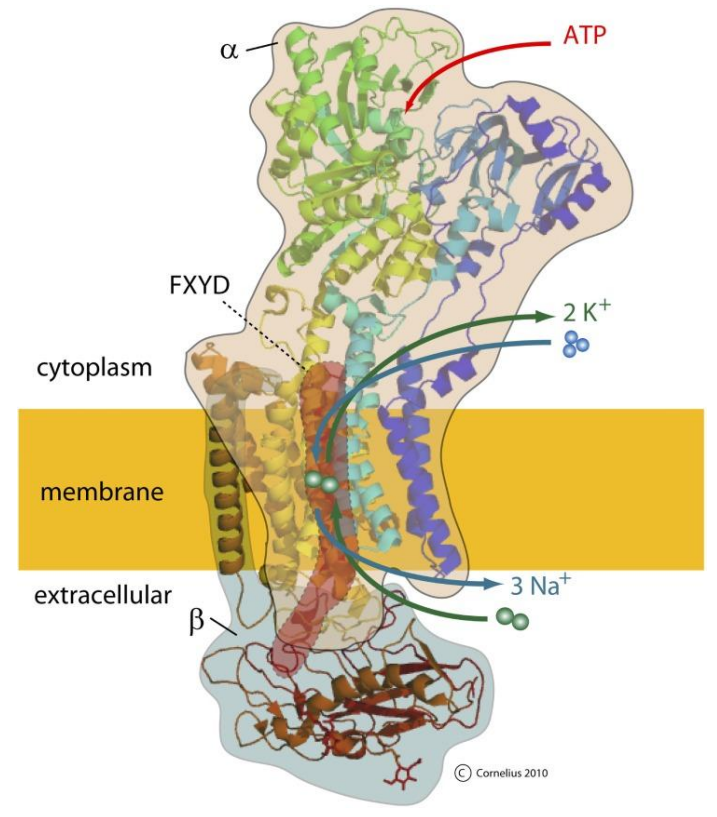

Figure 7. Structure of the $\mathrm{Na} / \mathrm{K}-\mathrm{ATPase}$. The NKA consists of an intracellular $\alpha$ - SU (encircled in light brown) and extracellular $\beta$-SU (encircled in blue). The applied color on the quaternary changes gradually from the $\mathrm{N}$-terminal (blue) to the C-terminal (red). Having a great transmembrane domain, the regulatory subunit of the pump (FXYD1-PLM) is red and associated with the a SU. The transmembrane domain contains binding sites for $3 \mathrm{Na}^{+}$and $2 \mathrm{~K}^{+}$ions, respectively, which pass sequentially through the same cavity of the molecule. Taken from Clausen et al. ${ }^{152}$. 
(see section 1. 3. 1) ${ }^{180,181,183}$. It is proposed that the different $\alpha$ isoforms vary in subcellular distribution and function. Berry et al. have previously shown that, the NKA with the $\alpha_{1}$ SU $\left(\mathrm{NKA}-\mathrm{a}_{1}\right)$ is more to be found on surface membrane compartments and provides around $88 \%$ of the total $\mathrm{Na}^{+}$current. Even though the NKA- $\alpha_{2}$ has a high functional T-tubular density being five times more present in the cardiac T-tubules than in the sarcolemma, the NKA- $\alpha_{1}$ still generates at least $50 \%$ of cardiac T-tubular $\mathrm{Na}$ current in mice ${ }^{184}$. Referring to their differential distribution in addition to their clear physical and functional association with the NCX ${ }^{185-187}$, the $\alpha_{1}$ and $\alpha_{2}$-SUs are suggested to have different functional roles in cardiac myocytes: The NKA- $\alpha_{1}$ is responsible for global $\mathrm{Na}^{+}$current, as it is the predominant isoform of the two, simultaneously it controls subsarcolemmal $\mathrm{Na}^{+}$microdomains together with the $\mathrm{NKA}-\alpha_{2}$ especially at the dyadic cleft located at the sarcoplasmatic reticulum ${ }^{166,188}$. Also, it is stated that the $\alpha_{2}$ isoform preferentially regulates cardiac $\mathrm{Ca}^{2+}$ transients via the $\mathrm{NCX}^{189-191}$. There is the intriguing idea of microdomains created by co-localization of ion transporters, where the NKA is regulated by $\mathrm{PLM}^{75,192,193}$. It is supported by the fact that there is an indirect influence of the NKA isoforms on sub-sarcolemmal $\mathrm{Na}^{+}$controlled by the NCX which has already been suggested to be functionally linked to the NKA ${ }^{192}$. Additionally, it is stated that the cytoskeletal protein ankyrin B anchors NCX to the membrane actively forming a functional complex with the NKA and the IP3-receptor supports ${ }^{75,193}$.

\section{3 Kinase mediated control of Phospholemman over the $\mathrm{Na}^{+} / \mathrm{K}^{+}$-ATPase}

\section{3. 1 Phospholemman- FXYD1 Protein}

As stated above, the NKA is regulated by different cellular mechanisms, for example by sodium and potassium levels or membrane potential as well as factors like ATP availability and cardiac steroid binding. Next to this, it is regulated by FXYD1, a small membrane protein belonging to a family of proteins with 7 identified members (FXYD1-7) being firstly defined as regulators for ion transporters and named according to the aforementioned signature FXYD motif in the $\mathrm{N}$-terminus ${ }^{182}$ (Figure 8). At least five of these proteins are described as auxiliary subunits regulating NKA activity in a tissue- and isoform-specific way ${ }^{194}$. FXYD1, also known as phospholemman (PLM), is a 72 amino acids long single transmembrane protein, predominantly found in heart, skeletal and smooth muscle cells ${ }^{195-198}$. The N-Terminus with its 17 amino acid residues lies in the extracellular domain, whereas the C-Terminus resides in the cytosolic fraction with 35 amino acids. Bearing multiple phosphorylation sides at the $\mathrm{COOH}$ terminus PLM is phosphorylated at the Ser68 residue by PKA and PKC ${ }^{199,200}$. The latter has additional phosphorylation targets at the Ser63 and Ser/Thr69 residues ${ }^{174}$. Physical association of PLM was demonstrated by co-immunoprecipitation with the $\alpha-S U$ of the NKA ${ }^{76}$ and supported by FRET experiments by Khafaga et al. ${ }^{201}$. Worthy of comparison is PLB, 
which is also a PKA substrate whereupon the SERCA2a is disinhibited, but PLB does not alter the maximum transport rate $\left(\mathrm{V}_{\max }\right)$ of its pump like PLM does for NKA. Several laboratories have shown that PLM phosphorylation relieves the sodium pump by possibly increasing $\mathrm{V}_{\max }{ }^{174,202-204}$ and $\mathrm{K}_{\mathrm{m}}$ for $\mathrm{Na}^{+}$ions (increased $\mathrm{Na}^{+}$affinity) ${ }^{15,76,200,205,206}$. Variation between studies regarding $\mathrm{V}_{\max } / \mathrm{K}_{\mathrm{m}}$ showed discrepancies most likely due to different methodology performed in different labs.

Several other functional roles are ascribed to PLM in the heart ${ }^{166}$. It is suggested that PLM modulates the gating of L-type Ca channels (Cav1.2) in an overexpression mode ${ }^{207}$. Another study on cardiac cells revealed that PLM oligomerizes ${ }^{208}$, as originally proposed but instead of forming ion channels in Xenopus oocytes ${ }^{209}$ and regulating cell volume in cultures cardiac cells by forming taurine efflux channels ${ }^{210}$, it is rather accepted to oligomerize ${ }^{199}$ in order to generate a 'storage pool' to increase 'responsiveness' of a certain PLM-free NKA population in the heart to adrenergic stimulation, by as yet unidentified means ${ }^{166}$.

Under catecholamine stress PLM works against of arrhythmogenesis although minimally causing negative inotropy by promoting $\mathrm{Na}$ extrusion ${ }^{211,212}$. For this reason PLM expression and its phosphorylation may be important for protection against stress-induced arrhythmia.

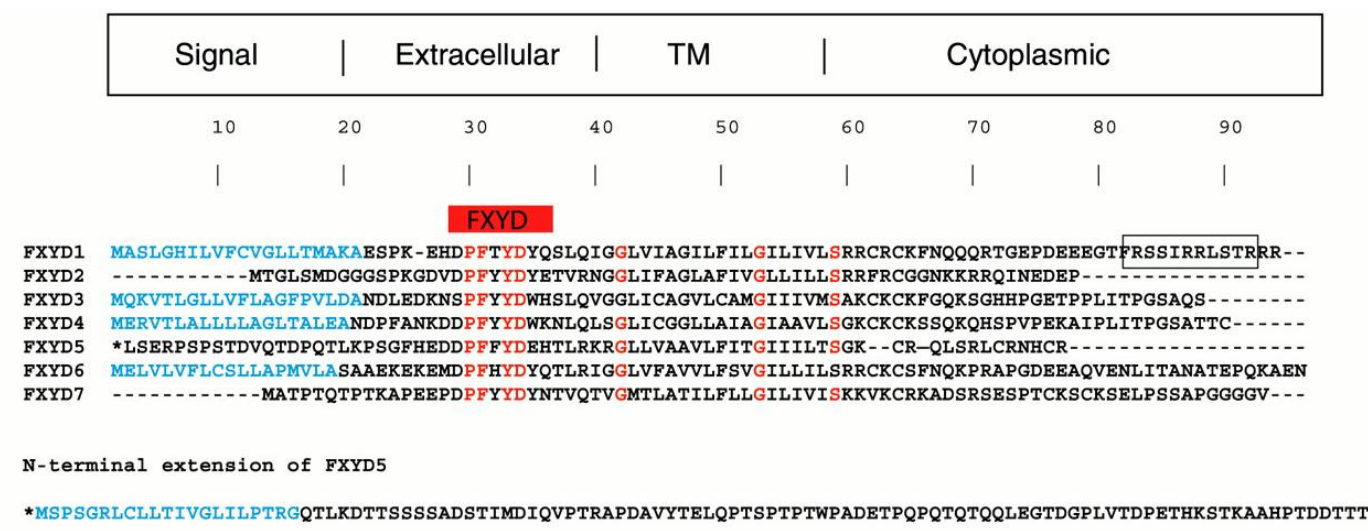

Figure 8. Amino acid sequence of currently known mammalian FXYD proteins.

Human FXYD sequence alignments showing seven known families with conserved residues (in red). The PKA/PKC phosphorylation domain of phospholemman is framed. Taken from Cornelius et al. ${ }^{182}$

\section{3. 2 Kinase phosphorylation of Phospholemman}

In 1985 PLM was identified as a PKA and PKC phosphorylatable protein in the heart ${ }^{213,214}$. Initially, it was assumed that the NKA is regulated through phosphorylation of its $\alpha$-SU via PKA, but it was strictly dependent on a detergent which makes the PKA phosphorylation site of the pump accessible ${ }^{215}$. It is now generally accepted that phosphorylated PLM activates the cardiac sodium pump and tonically inhibits it at its basal state ${ }^{76}$. Conclusive analysis of the $\mathrm{Na}^{+}$pump regulation was performed by whole cell voltage clamping of PLM-WT and 
PLM-KO cardiomyoctes showing pump activity mechanisms under $\beta$-AR stimulated and non-stimulated conditions $^{216}$. The $\beta$-AR stimulation of PLM-WT cells increased $\mathrm{Na}^{+}$pump activity and $\mathrm{Na}^{+}$affinity to levels of NKA current of PLM-KO cells, where there was no inhibitory effect of PLM to the NKA enzyme.

Additional

experiments introducing a

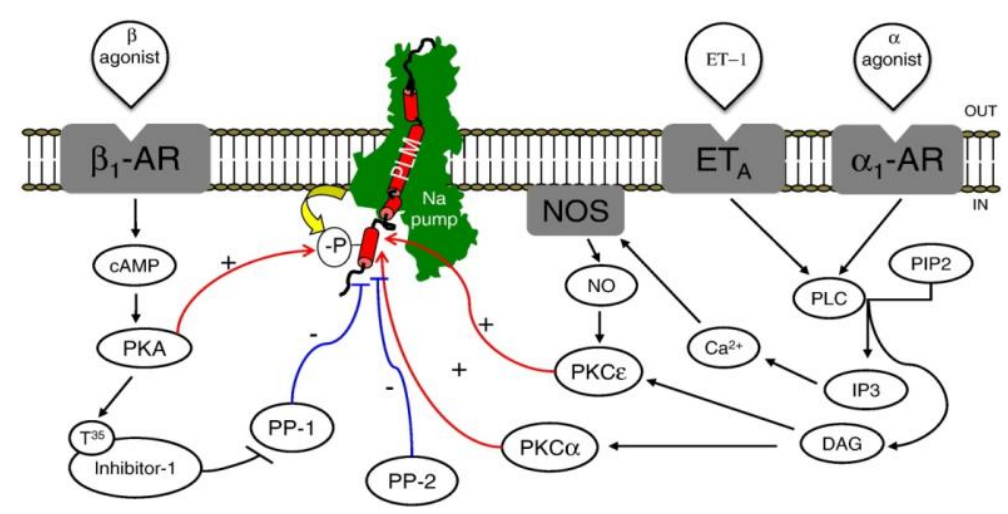

Figure 9. Phosphorylation of phospholemman regulates sodium pump activity. Signaling pathways mediating kinase and phosphatase activities involved in the PLM phosphorylation state. PKA and PKC phosphorylate PLM, hence stimulate the sodium pump, whereas PP-1 and PP2-A activity inhibit the pump by dephosphorylating PLM. ET-1, endothelin 1; $E T_{A}$, endothelin $A$ phosphorylated PLM peptide to receptor; NOS, nitric oxide synthase. Taken from Pavlovic et al. ${ }^{173}$

cardiomyocytes of the same animals have indicated that Ser68 phosphorylation of PLM can even stimulate $\mathrm{Na}^{+}$pump current above basal levels, simultaneously indicating that the final 19 amino acids of PLM are sufficient to inhibit the NKA ${ }^{202}$. Phosphorylation of PLM leads to a change in orientation, but the FXYD1 protein remains physically associated to the $\alpha_{1}$-SU of the cardiac sodium pump, which is co-immunoprecipitated in several studies ${ }^{76,171,217,218}$. It is widely known that similarly to PLM dependent PKA activation of the $\mathrm{Na}^{+}$pump, protein kinase $C$ (PKC) mediated phosphorylation of PLM increases $V_{\max }$ with a change in $\mathrm{Na}^{+}$ affinity of the NKA in some cases ${ }^{172,200}$. There are speculations that PKC acts on a different pool of PLM predominantly associated to the $\alpha_{2}$ isoform of the pump. The pump isoform specific effect of PLM phosphorylation via PKC is a theory demonstrated in several experiments from different labs ${ }^{172,205}$ which still remains questionable. It is supported by the fact that for instance PKC phosphorylation increases the maximum rate of the $\alpha_{2}$-NKA but not $\alpha_{1}$-NKA in genetically modified SWAP mice (where $\alpha_{1}$-NKA can be blocked by ouabain), and contradicted by FRET experiments showing no actual change between YFP-PLM and CFP$\alpha_{1}$ and $-\alpha_{2}$, respectively ${ }^{205}$. PLM phosphorylation at its multiple target residues by PKA and PKC turns out to be additive ${ }^{200}$, also in terms of NKA function. Investigations in ARVMs have shown high basal phosphorylation of PLM ( 30\% at Ser68 and $\sim 50 \%$ at Ser63) mainly caused by the PKC activity, hence basal $\mathrm{Ca}^{2+}$ levels ${ }^{174}$. Three PKC isoforms are identified in the heart $(\mathrm{PKC \alpha}, \delta \text {, and } \varepsilon)^{174}$. Fuller et al. demonstrated that receptor-mediated PKC activation causes sustained phosphorylation of Ser63 and Ser68, but transient phosphorylation of Thr69, which leads to further stimulation of the sodium pump ${ }^{174}$. There is evidence that exogenous/endogenous NO mediates PKC $\varepsilon$ dependent PLM phosphorylation thereby limiting $\mathrm{Na}^{+}$and $\mathrm{Ca}^{2+}$ overload and related cardiac diseases ${ }^{219}$. 
The kinase phosphorylation of phospholemman, in particular during sympathetic stimulation is essential for the $\mathrm{ECC}^{17}$ where the phosphoprotein disinhibits NKA ${ }^{15,203}$ (Figure 9) while actively inhibiting the $\mathrm{NCX}^{173,220}$ thereby regulating intracellular $\mathrm{Ca}^{2+}$ concentration critical for cardiac function ${ }^{221}$.

\section{4 Changes of PLM/ NKA complex in diseased heart}

The importance of NKA function in the heart in terms of trans-membrane sodium gradient is demonstrated by several laboratories (see section 1. 2. 2). It is well known that $\mathrm{Na}^{+}$overload contributes to cardiac diseases such as ischemia/reperfusion ${ }^{222,223}$ hypertrophy and heart failure $(\mathrm{HF})^{224-226}$. Elevation in intracellular $\mathrm{Na}^{+}$which occurs largely through several sodium influx pathways shown to be up-regulated in the failing myocardium ${ }^{227,228}$, may contribute to the negative force-frequency relationship, slowed relaxation and arrhythmias ${ }^{190}$. A large body of data suggests that this may be caused by a reduction of NKA activity and compromised $\mathrm{Na}^{+}$extrusion via the pump in heart failure ${ }^{229,230}$. Additionally, aberrant phosphorylation of PLM $^{231}$, the expression of NKA and its $\alpha$ subunits contribute to the disease pattern ${ }^{217}$. There are different studies by groups focused on different stages of the disease process in human, canine and rat heart failure models ${ }^{226,232,233}$. For example, Bossuyt et al. showed that the PLM fraction phosphorylated at Ser-68 was dramatically increased in a rabbit HF model ${ }^{217}$. Comparable results were obtained in studies on murine cells 3 weeks post-Ml by Mirza et al. This group observed a decrease in PLM expression, accompanied by an increase in the fraction of phosphorylated PLM in murine hearts 3 weeks post-MI ${ }^{234}$. Additionally, the enhanced NCX activity in PLM-KO-MI cells largely accounted for the improvement in cardiac and myocyte contractility compared to WT-MI hearts ${ }^{234}$. El-Armouche et al. reported significantly reduced PLM phosphorylation, which could possibly be involved in disease progression linked to an increase in Inhibitor-1 and Protein Phosphatase-1 (PP-1) activity, but there was no change in expression for total PLM or the $\alpha_{1}$-SU in failing human hearts ${ }^{231}$. Similarly, data presented by Boguslavskyi et al. clearly indicate that the complete prevention of PLM phosphorylation sites via a PLM mutant knock-in mouse subjected to aortic constriction markedly exacerbates cardiac remodeling by further inhibiting the $\mathrm{NKA}^{230}$. Pointing out the aforementioned preferential role of the $\alpha_{2}$-NKA in EC coupling (section 1. 1. 2 ), the same group ${ }^{230}$ and others ${ }^{235}$ have revealed that the increase in $\alpha_{2}$-SU expression is a positive adaptive mechanism possibly allowing the normalization of ion, especially $\mathrm{Ca}^{2+}$, regulation in response to $\mathrm{Na}^{+}$overload caused by hypophosphorylation of PLM in animals with induced hypertrophy. 


\section{5 Imaging local cyclic nucleotide dynamics}

\section{5. 1 Fundamentals of Förster/Fluorescence resonance energy transfer}

As mentioned in section 1. 1. 3 , it is widely accepted that spatial and temporal compartmentation of CAMP is important for targeting a range of proteins in functionally important subcellular locations. Measuring second messenger levels via biochemical assays such as radioimmunoassays directly detecting cAMP content in a cell pellet ${ }^{236-238}$ or indirect immunoblot analysis of phosphorylated proteins by the respective kinases (i.e. PKA) are alternative ways used to measure cAMP concentrations ${ }^{239}$. Offering poor spatial resolution, these classical methods do not enable to obtain results of physiological relevant free cAMP effects but rather total amounts of cAMP in lysed tissue, not focusing on single cells let alone cellular compartments. As most cellular activities appear in milliseconds, there was an urge to gain insights into cAMP dynamics at high temporal resolution.

Most commonly used live cell imaging techniques are based on the Förster resonance energy transfer (FRET) phenomenon, discovered by the German physicist Theodor Förster in $1948^{240}$. The Förster equation $E(F R E T)=1 /[1+(r / R o)]^{6}$ describes the non-radiative energy transfer between two fluorophores in very close proximity $(<10 \mathrm{~nm})$ which has provided a popular mean to detect protein-protein interaction and conformational change of proteins in living cells. More precisely, the donor fluorophore is excited with a specific wavelength of light matching its peak of absorption. The excited donor in turn transfers its emission energy to the acceptor fluorophore (Figure 10A). Therefore, a FRET pair should necessarily consist of two fluorophores, by which the donor fluorophore emission spectrum should overlap with the excitation spectrum of the acceptor fluorophore ${ }^{241}$. The well-known Jablonski diagram (Figure 10B) demonstrates in detail the excited $\mathrm{S}_{1}$ state of the donor electron and the its fall back to ground state $S_{0}$ via emission of fluorescence energy in form of a photon exciting the acceptor fluorophore on a energetically lower level ${ }^{242}$. Similar to its donor pair, the excited acceptor electron emits fluorescent light when returning to its singlet ground state $S_{1}$. According to the change in energy state, the donor fluorescence (emission) intensity is reduced and the acceptors emission is increased. For quantification of FRET occurring between the pair of fluorophores, the ratio acceptor emission intensity/donor emission intensity or vice versa is calculated, and a change in FRET can be observed during experiments $^{239}$. FRET pairs often consist of green fluorescent protein (GFP) mutants, such as cyan fluorescent protein (CFP) as the donor protein and yellow fluorescent protein (YFP) as the acceptor protein. Tagged to sequences of proteins of interest, these fluorophores allow through optical recordings to monitor dynamic protein-protein interactions ${ }^{243}$ (Figure 10C) ${ }^{171,201}$. FRET between CFP and YFP sandwiching cyclic nucleotide binding domains was introduced as a tool to detect cAMP and cGMP dynamics in several studies mentioned in the next section. 
A

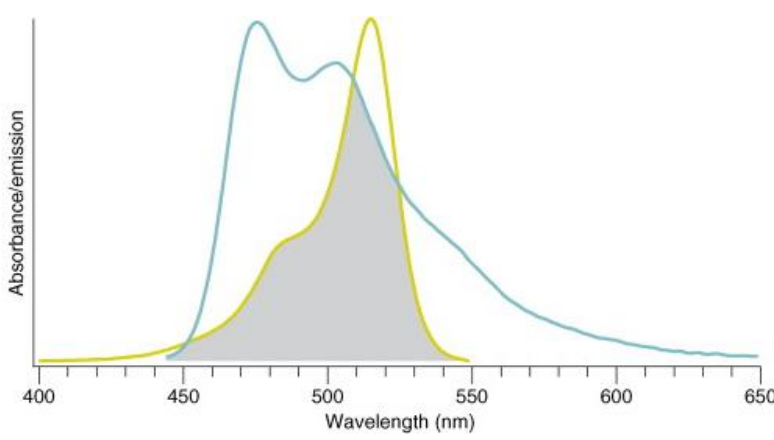

B

S1

so

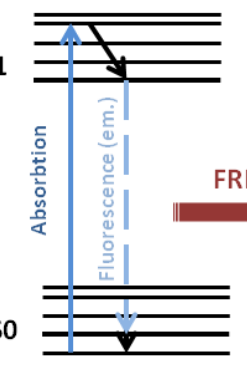

S1

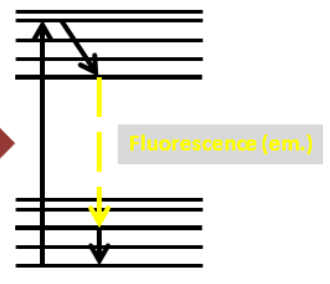

C
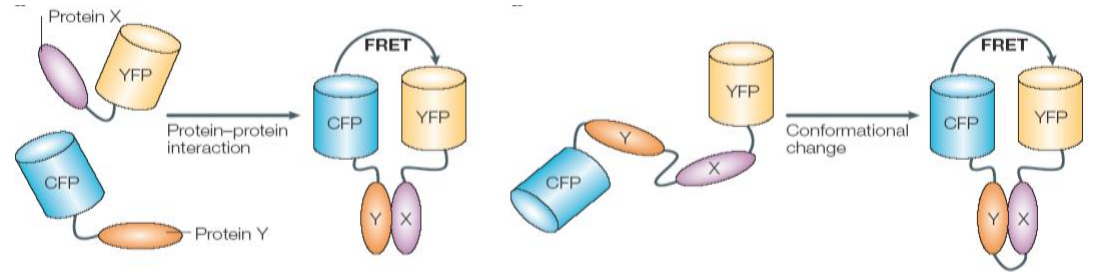

Figure 10. The principles of FRET instancing the CFP and YFP pair. (A) The prerequisite for FRET to occur is a spectral overlap between the donor and acceptor fluorophore. The emission spectrum (blue) of the donor fluorophore (CFP) overlaps with the excitation spectrum (yellow) of the acceptor fluorophore (YFP) and allows fluorescence resonance energy transfer (FRET). The overlapping spectral area is colored grey. Taken from Piston et al. ${ }^{225}$ (B) The schematic representation of the Jablonski-diagram describes the transfer of fluorescence energy from the donor to the acceptor fluorophore. Upon absorption of photon energy, the donor electron moves from a ground state $(\mathrm{S} 0)$ to its excited state (S1). The donor fluorophore can partly transfer its energy from the lowest $\mathrm{S} 1$ state in form of fluorescent energy. The electrons of the acceptor fluorophore with a lower energetic excitation spectrum and close proximity is excited. Acceptor electrons are lifted from their S0 to the S1 state and yellow fluorescence light is emitted when the acceptor electrons "fall back" into their S0 state. ${ }^{226}$ (C) FRET is utilized for tracking dynamic protein-protein interactions and conformational changes within a single protein. Taken from Zhang et al. ${ }^{227}$

\section{5. 2 cAMP sensitive FRET Biosensors}

Before starting to generate (targeted) CAMP sensitive FRET based biosensors, several groups have used the change in cAMP dependent $\operatorname{LTCC}^{70}$ and $C N G C^{102,244,245}$ currents as a readout for local CAMP dynamics and significantly contributed to characterization of $\beta_{2}$-ARcAMP pools shaped by PDEs (i.e. PDE3 and PDE4) ${ }^{246}$. Adams et al. created the first chemically labeled cAMP sensitive sensor called FliCRhR, utilizing the regulatory and catalytic subunits of the PKA holoenzyme. Injecting it to living cells, cAMP activity is monitored through PKA dissociation and loss of $F R E T^{247}$. The more practical genetically encoded PKA based biosensor was developed by Zaccolo et al., who fused CFP and YFP to the $\mathrm{R}$ and $\mathrm{C}$ subunits to detect CAMP changes close to the Z-lines in neonatal rat ventricular myocytes $^{72,248}$. It was the first attempt to monitor distinct PDE activities in subcellular microdomains. This sensor showed slow kinetics and probable interaction with endogenous PKA (Figure 11). Subsequently, several single cAMP binding site based sensors were 
developed, which can monitor cAMP in the bulk cytosol. For example, the HCN2-camps sensor ${ }^{28}$ contained the soluble C-terminal cAMP binding domain from the cyclic nucleotidegated potassium channel 2 flanked with CFP and YFP sites enabling real time measurements of cAMP dynamics in the bulk cytosol. Additionally, either Epac1 or Epac2 ased single chained biosensor with uniform cellular expression were widely used to detect rapid cAMP signals developing in the cytosol ${ }^{249,250}$. Fused to CFP and YFP, a single cAMP binding domain changes its conformation upon cAMP leading to a decrease of the FRET signal. Epac1 and Epac2 based sensors differ in their cAMP affinity and FRET capacity. Despite its lower cAMP affinity, Epac1 based sensor E1-camps showed a larger FRET change, which is why it is used in this and other studies ${ }^{154,239,251}$.

A

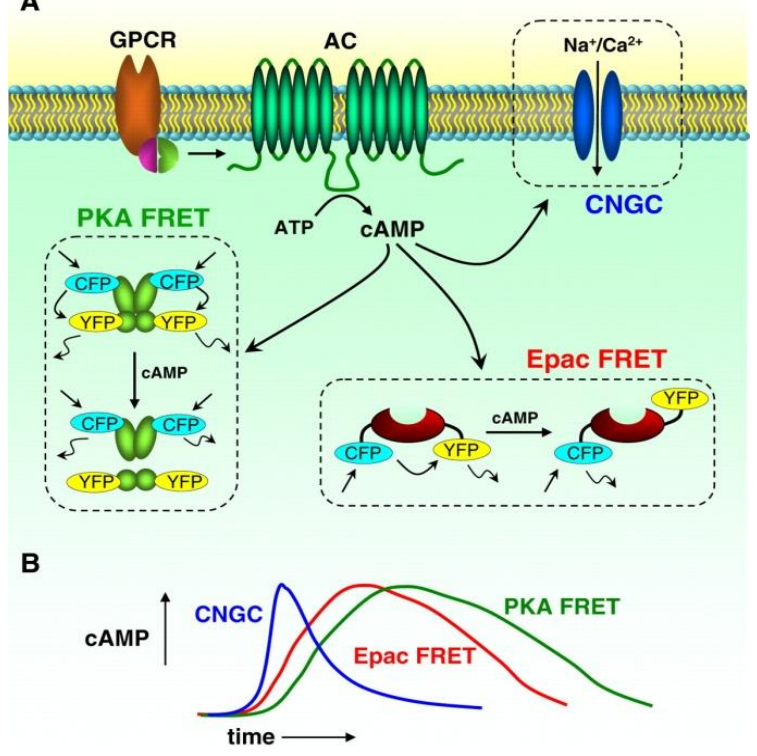

Figure 11. Cartoon showing means to determine intracellular cAMP dynamics. (A) Regulatory and catalytic subunits of PKA probes dissociate upon CAMP binding causing a decrease in the FRET signal. The Epac based FRET sensor has CFP and YFP in close proximity at basal state, after cAMP binds to the unimolecular sensor, there is a conformational shift and FRET-based YFP emission decreases. CAMP signals can be measured through determination of cyclic nucleotide gated channel currents by patch-clamp methods, or by imaging with a $\mathrm{Ca}^{2+}$-sensitive dyes. (B) Compared with PKA-based probes, the Epac-based sensors exhibit an extended dynamic range and have better signal-to-noise ratio. FRET changes detected with the CNGC method are more transient and have a greater temporal resolution although they are restricted to the membrane. Taken from Willoughby et al. ${ }^{229}$ 


\section{6 Aims of the project}

Previous studies have demonstrated that several cAMP-dependent PKA substrates important for the regulation cardiac ECC are organized in microdomains formed by scaffolding proteins and $\beta$-adrenergic receptor subtypes. However, the knowledge about the real time dynamics of CAMP in a microdomain coupled to PLM and NKA, its interaction with $\beta$-ARs and individual cardiac PDE families forming this important microdomain as well as their alterations in cardiac disease is insufficient.

Therefore, the aims of this work were:

1. To develop a FRET-based Phospholemman targeted cAMP biosensor PLM-E1 and to express it in ARVMs using an adenoviral expression system.

2. To perform real time monitoring of CAMP dynamics in the PLM/NKA compartment in order to understand the regulatory mechanisms behind a possible local restriction of $\beta$-AR mediated cAMP signals in the PLM/NKA microdomain.

3. To study microdomain-specific alterations of cAMP signaling at the PLM/NKA complex in a chronic heart failure disease model. 


\section{Materials and Methods}

\section{1 Materials}

\section{1. 1 Cells}

HEK293A

Invitrogen

HEK293

Sigma Aldrich

\section{1. 2 Plasmids}

pcDNA3.0

Epac1-camps

human PLM

canine PLM-YFP

human PLM-E1 long linker

canine PLM-E1 short linker

Gateway® pDONR ${ }^{\text {TM }} 221$

Gateway ${ }^{\circledR}$ pAd/CMV/V5-DEST ${ }^{\mathrm{TM}}$
Invitrogen

Prof. Viacheslav Nikolaev

Prof. Michael Shattock

Dr. William Fuller

this project

this project

Invitrogen

Invitrogen

\section{1. 3 Bacteria strains}

One Shot@ TOP10 chemically competent E. coli Invitrogen One Shot® OmniMax2T1 Invitrogen

\section{1. 4 Animals}

Adult male 8-12 weeks old Wistar rats (250 - 300 g; Charles River) were held on site (Animal Care Facility, UMG Göttingen). They were maintained in compliance with the constitutional animal protection act (Tierschutzgesetz, TSchG). All animals were kept in containment rooms at room temperature. Between 4 and 6 rats were housed in each cage, allowing free access to food and water. All animal protocols were approved by the local University Medical Center Göttingen animal protection committee and all animal work was performed according to institutional and governmental guidelines.

Adult male 8 weeks old Sprague Dawley rats ( $200 \mathrm{~g}$ ) were used for introduction of the heart failure rat model and underwent heart isolation 16 weeks post MI, as described in sections 2. 2. 18 and 2. 2. 19. Aged matched control animals (AMC) were ordered both at our site in Göttingen (Janvier Labs) and at the Imperial College London in order to use for control experiments. 


\section{1. 5 Oligonucleotides}

The human PLM sequence was kindly provided by Prof. Michael Shattock, King's College London.

human PLM sequence

5'-ATGGCGTCTCTTGGCCACATCTTGGTTTTCTGTGTGGGTCTCCTCACCATGGCCAAGGCAGAAAGTCCAAAGGAACACG ACCCGTTCACTTACGACTACCAGTCCCTGCAGATCGGAGGCCTCGTCATCGCCGGGATCCTCTTCATCCTGGGCATCCTCA TCGTGCTGAGCAGAAGATGCCGGTGCAAGTTCAACCAGCAGCAGAGGACTGGGGAACCCGATGAAGAGGAGGGAACTTT CCGCAGCTCCATCCGCCGTCTGTCCACCCGCAGGCGG-3'

The canine PLM-YFP sequence with linker sequence (60 bp) was provided by Dr. William Fuller.

canine PLM-YFP

5'-ATGGCACCTCTCCACCACATCTTGGTTCTCTGTGTGGGTTTCCTCACCACGGCCACCGCAGAAGCGCCACAGGAACA CGACCCGTTCACCTACGACTACCAATCCCTGCGGATCGGAGGCCTCATCATCGCCGGGATCCTCTTCATCCTCGGTATCCT CATCGTCCTGAGCAGAAGATGCCGGTGCAAATTCAACCAGCAGCAGAGGACTGGGGAACCTGATGAAGAGGAGGGAACTT TCCGCAGCTCCATCCGCCGTCTGTCCACCCGCAGGCGGAAGAGATCTCGAGCTCAAGCTTCGAATTCTGCAGTCGACGGT ACCCCGGTCGCCACCGGGGTGAGCAAGGGCGAGGAGCTGTTCACCGGGGTGGTGCCCATCCTGGTCGAGCTGGACGGC GACGTAAACGGCCACAAGTTCAGCGTGTCCGGCGAGGGCGAGGGCGATGCCACCTACGGCAAGCTGACCCTGAAGTTCA TCTGCACCACCGGCAAGCTGCCCGTGCCCTGGCCCACCCTCGTGACCACCTTCGGCTACGGCCTGATGTGCTTCGCCCG CTACCCCGACCACATGAAGCAGCACGACTTCTTCAAGTCCGCCATGCCCGAAGGCTACGTCCAGGAGCGCACCATCTTCTT CAAGGACGACGGCAACTACAAGACCCGCGCCGAGGTGAAGTTCGAGGGCGACACCCTGGTGAACCGCATCGAGCTGAAG GGCATCGACTTCAAAGAGGACGGCAACATCCTGGGGCACAAGCTGGAGTACAACTACAACAGCCACAACGTCTATATCATG GCCGACAAGCAGAAGAACGGCATCAAGGTGAACTTCAAGATCCGCCACAACATCGAGGACGGCAGCGTGCAGCTCGCCG ACCACTACCAGCAGACACCCCCATCGGCGACGGCCCGTGCTGCTGCCGACACACTACTGAGCTACAGTCCGCCC-3' Which includes the canine PLM, the linker and the YFP sequences

All other oligonucleotides were purchased from MWG Biotech $\mathrm{GmbH}$, eurofins Genomics, Ebersberg:

PLMHindlllfor

5'-AAAAAGCTTACCATGGCGTCTCTTGG-3'

PLMNhelrev

5'-AAAGCTAGCAGATCCGGATCCCCGCCTGCGGGTG-3'

YFPNhelfor

5'-AAAGCTAGCGTGAGCAAGGGCGAGG-3'

PLMYFPKpnfor

5'-AAAGGTACCCCGGTCGCCACCGGGGTGAGCAAGGGCGAGG-3'

YFPEcoRIrev

5'-AAAGAATTCCTTGTACAGCTCGTCCATG-3'

pcDNA3attBfor

5'-GGGGACAAGTTTGTACAAAAAGCAGGCTGACTCACTATAGGGAGACCC-3'

pcDNA3attBrev

5'- GGGGACCACTTTGTACAAGAAAGCTGGGTAGCGAGCTCTAGCATT-3' 
attBPLMhundfor

5'-GGGG ACA AGT TTGTACAAAAAAGCAGGC TACGATGGCACCTCTCC-3'

attBYFPN1 rev

5'-GGGGACAAGTTTGTACAAAAAAGCAGGCTCCGCGACTCTAGATCA-3'

\section{1. 6 Chemicals}

Acrylamide (Rotiphoreses Gel 30)

ANP

Ampicillin

Ampuwa® water

BAY 60-7550

$\beta$-Mercaptoethanol

Bromphenol Blue sodium salt

8-Br-2'-O-Me-cAMP-AM

2,3- Butanedione monoxime

Bumetanide

Calcium chloride

Calcium chloride dihydrat

CGP-20712A methanesulfonate salt

Cilostamide

Dimethyl sulfoxide DMSO

dNTPs

EDTA

Ethanol Rotipuran $>99,8 \%$

EZ-Link $^{T M}$ Sulfo-NHS-SS-Biotin

Forskolin

GFP-Trap® A

Glucose

Glycerol

Glycine

$\mathrm{H}-89$ dihydrochloride hydrate

HEPES

Hydrochloride acid 37\%

ICl-118.551 hydrochloride

3-Isobutyl-1-methylxanthin

Isoproterenol hydrochloride

Laminin
(ROTH)

(Bachem)

Roth, \# K029.1

Fresenius Kabi Deutschland $\mathrm{GmbH}$

Santa Cruz, \# sc-205219

Sigma, \# M3148

Applichem, \# A1120

Biolog, \# B028

Sigma, \# B0753

Sigma, \#B3023-250MG

Sigma, \# C4901-1KG-D

Merck, \# 17257

Sigma, \# C231

Sigma, \# C7971

Sigma, \# D2650

Promega, \# U1240

Roth, \# 8040.3

Roth, \# 9065.1

Thermo Fischer, \# 21331

Sigma, \# F6886

Chromotech, \# gta-10

Sigma, \# G7021

Sigma, \# G8773

Roth, \# 3908.3

Sigma, \# B1427

Sigma, \# H4034

Sigma, \# 84422

Sigma, \# 1127

Applichem, \# A0695

Sigma, \# 16504

Sigma, \# L2020 
LB- Agar powder Miller

LB- Medium powder Miller

Loading buffer DNA IV (for Agarose gels)

Kanamycin

Magnesium chloride

Magnesium chloride hexahydrate

Magnesium sulfate heptahydrate

MDL-12,330A hydrochloride

Methanol

8-methoxymethyl-3-isobutyl-1-

methylxanthine

Milkpowder

Monensin sodium salt

$\mathrm{N}$-Methyl-D-glucamine

$N, N, N^{\prime}, N^{\prime}$-Tetramethylethylenediamine

(TEMED)

Octaethylene glycol monododecyl ether

Ouabain octahydrate

peqGOLD Universal Agarose

PhosStop

Ponceau S

Potassium bicarbonate

Potassium chloride

Potassium dihydrogen phosphate

Protease Inhibitor Cocktail

Protein G Sepharose 4 Fast Flow

Protein Marker $\mathrm{V}$

Quick-Load® 100bp DNA ladder

Quick load® 1 kb DNA ladder

Rolipram

Rubidium-86 Radionuclide

Sodium azide

Sodium bicarbonate

Sodium chloride

Sodium hydroxide

Streptavidin Sepharose High Performance

TAE-buffer (50x)
Applichem, \# A0927

Applichem, \# A0954

Applichem, \# A3481

Applichem, \# A1493

Sigma, \# M8266-1KG

Applichem, \# A1036

Sigma, \# M2773

Sigma, \# M182

Roth, \# HN41.2

Sigma, \# M2547

Roth, \# T145.1

Sigma, \# M5273-1G

Sigma, \#66930-100G

Sigma, \# T9281

Sigma

Sima, \# O3125-1G

Peqlab, \# 35-1020

Roche, \# 04906837001

Sigma, \# P3504

Sigma, \# P7682

Sigma, \# P5405

Merck, \# 4873

Roche, \# 11872580001

GE Healthcare, \#17-0618-01

Peqlab, \#27-2211

Biolabs, \# NO467S

Biolabs, \# NO468S

Sigma, \# R6520

Perkin Elmer, \# NEZ072001MC

Sigma, \# S2002

Sigma, \# S5761

Sigma, \# S5886

Roth, \# 6771.3

GE Healthcare, \# 17-5113-01

Applichem, \# A1691 
Taurine

TRIS

Triton- $X ® 100$

Tween- $20 \AA$

Vectashield® Mounting Medium
Sigma, \# T8691

Roth, \# 4855.3

Applichem, \# A1287.0025

Sigma, \# P1379

Vector Laboratories, \# H-1000

\subsection{Cell culture}

Geneticin® Selective Antibiotic

(G418 Sulfate) (50 mg/mL)

Thermo Fischer \#10131027

DMEM, $4.5 \%$ glucose

Biochrom, \# F0445

FCS

Biochrom, \# S0615

Glutamine

Biochrom, \# K0283

Iscove Basal Medium

Biochrom, \# FG 0465

Lipofectamine $\AA 2000$ Reagent

Modified Medium 199

Invitrogen, \# 11668

OPTI-MEM®

Sigma, \#M7528

Gibco, \# 11058

PBS Phosphate Buffered Saline

(Dulbecco)

Biochrom, \# L1825

Penicillin/Streptomycin

Biochrom, \# A2213

Plaque GP Agarose

Biozym, \# 850110

Trypsin/ EDTA solution

Biochrom, \# L2143

\section{1. 8 Enzymes and Kits}

Pfu DNA Polymerase (2-3 U/ $\mu \mathrm{L})$

Promega, \# M774B

Restriction enzymes for molecular cloning

New England Biolabs

Pierce BCA Protein Assay Kit

Thermo Scientific, \# 23227

Plasmid Midi Kit

Qiagen, \# 12945

Plasmid Mini Kit

Qiagen, \# 12125

Proteinase $\mathrm{K}$

Applichem, \# A3830-0500

QIAquick Gel Extraction Kit

Qiagen, \# 28704

QIAquick PCR purification Kit

Qiagen, \# 28104

T4 DNA Ligase

NEB, \# M0202S 


\section{1. 9 Antibodies}

Table 2. Primary antibodies are used for Western Blot (WB) and immunofluorescence (IF) analysis. Antibodies for Western Blot analysis were diluted in the used blocking buffer (TBS $+1 \%$ Tween $+5 \%$ milk powder) and for immunofluorescence experiments in appropriate blocking buffer (PBS $+10 \%$ FCS $+0.2 \%$ Triton-X-100).

\begin{tabular}{|l|l|l|}
\hline \multicolumn{1}{|c|}{ Antibody } & \multicolumn{1}{c|}{ Dilution } & \multicolumn{1}{c|}{ Company } \\
\hline anti- $\alpha_{1}$ mouse monoclonal C464.6 & $\begin{array}{l}\text { Western Blot: 1:10000 } \\
\text { Immunofluorescence: 1:100 }\end{array}$ & Millipore, \# 05-369 \\
\hline anti-FXYD1 (PLM) rabbit polyclonal & $\begin{array}{l}\text { Western Blot: 1:10000 } \\
\text { Immunofluorescence: 1:100 }\end{array}$ & Abcam, \# ab76597 \\
\hline anti-GFP rabbit monoclonal & Western Blot: 1:10000 & Abcam, \# ab32146 \\
\hline anti-PDE3A goat polyclonal & Immunofluorescence: 1:100 & Santa Cruz, \# sc11834 \\
\hline
\end{tabular}

Table 3. Secondary antibodies were diluted in buffers prepared for the used primary antibodies for WB and IH analysis.

\begin{tabular}{|l|l|l|}
\hline \multicolumn{1}{|c|}{ Antibody } & \multicolumn{1}{c|}{ Dilution } & \multicolumn{1}{c|}{ Company } \\
\hline Alexa Fluor® 488 donkey anti-goat & Immunofluorescence: 1:500 & Invitrogen, \# A11055 \\
\hline Alexa Fluor® 633 goat anti-rabbit & Immunofluorescence: 1:500 & Invitrogen, \# A21070 \\
\hline Alexa Fluor® 488 goat anti-rabbit & Immunofluorescence: 1:500 & Invitrogen, \# A11034 \\
\hline Alexa Fluor® 633 rabbit anti-mouse & Immunofluorescence: 1:500 & Invitrogen, \# A21063 \\
\hline $\begin{array}{l}\text { Immun-Star }{ }^{\text {TM }} \text { goat anti-mouse- } \\
\text { HRP }\end{array}$ & Western Blot: 1:5000 & Biorad, \# 170-5047 \\
\hline $\begin{array}{l}\text { Immun-Star }{ }^{\text {TM }} \text { goat anti-rabbit- } \\
\text { HRP }\end{array}$ & Western Blot: 1:5000 & Biorad, \# 170-5046 \\
\hline
\end{tabular}

\section{1. 10 Microscope devices and software}

Attofluor ${ }^{\circledR}$ cell chamber

AxioObserver A1 epifluorescence microscope

Axiovert 200 microscope

CFP/YFP filter set

CoolLED $440 \mathrm{~nm}$

CoolSNAP-HQ CCD-camera

DualView filter slider

ImageJ Software

Inverted fluorescent microscope

710 NLO microscope

Microsoft Office Picture Manager
Invitrogen

Carl Zeiss Microlmaging

Carl Zeiss Microlmaging

Chroma Technology

CoolLED

Visitron Systems

Photometrics

National Institutes of Health

Nikon

Carl Zeiss Microlmaging

Microsoft 
Corporation Oil immersion 63x objective

ORCA-03G camera

ZEN 2010 Software

\subsubsection{General devices and software}

Alphalmager® software

Biotek Reader (for BCA assay)

Centrifuges

MS-Excel

MS-PowerPoint

MS-Word

Multilmage Light Cabinet

NanoDrop 2000

Powerpac HC

Thermocycler

ThermoMix compact

Ultracentrifuge L-70

iCycler

Mini-PROTEAN® Electrophoresis System

Mupid-One Gel Electrophoresis

Origin Pro 8.5 Software

$\mathrm{pH}$ meter

TRI-CARB 4910TR

Ultracentrifuge L-70

UV Table IL-350-M

Ventilator Minivent

X-Ray Film processor

\section{1.12 Other materials}

Dialysis Tubing for virus dialysis

Eppendorf tubes

Cryogenic Vials, Nunc

Falcon tubes

Fiber pads for Western blot

Filter Unit 0.2 RC Spartan $130.2 \mu \mathrm{m}$

(DNA filtration)
Carl Zeiss Microlmaging

Hamamatsu Photonics

Carl Zeiss Microlmaging

ProteinSimple

BIOTEK Instruments

Thermo Scientific

Microsoft

Microsoft

Microsoft

Alpha Innotech Corporation

Thermo Scientific

Biorad

Sensoquest

Eppendorf

Beckman

Biorad

Biorad

Unit ADVANCE Co., Ltd.

OriginLab Corporation

Inolab

Perkin Elmer Inc., \# A491000

Beckman

Bachofer

Hugo Sachs Electronic

SRX 101A Konica

Medical International Ltd.

Eppendorf

Thermo Scientific, \#5000-0012

BD Falcon

Bio Rad, \#1703933

Whatman, \# 10463040 
Forene®

21-gauge needle

26-gauge needle

Gauze

Glass Cover Slips $24 \mathrm{~mm}$

Heparin 25000 I.E./5 ml

Microscope Slides

Medical X-Ray

Pony Vial

Prolene suture 6-0

Protran Nitrocellulose Transfer Membrane

Quickseal Centrifuge Tubes (virus centrifugation)

Scintilation Liquid Lumasafe Plus

Serological pipettes

Slide-A-Lyzer Dialysis Cassettes, 10K MWCO

Spacer Plates for Western blot

Steriflips

Short Plates for Western blot

Temgesic $\circledast$

U-40 Insulin 30Gx1/2

U-40 Insulin Omnifix Solo

Water bath

6 Well Plates

12 Well Plates

96 Well Plates

96 Well Plate for MicroBeta
Abbott

BD Microlane

BD Microlane

Th Geyer, \# 9.068291

Thermo Scientific, \# 004710781

Rotexmedica

Thermo Scientific, \# J1800AMNZ

Film Fujifilm, \# 40144037

Percin Elmer, Inc., \# 6000292

Ethicon

Whatman, \# 4018650

Beckmann, \# 342413

Lumac LSC, \# 3097

Sarstedt

Thermo Scientific, \# $663830.5 \mathrm{ml}$

Bio Rad, \#1653311

Millipore, \# SCGP00525

Bio Rad, \#1653308

Essex Pharma GmbH

Braun, \# 40012525

Braun, \# 9161309v

Julabo

Starlab, \# CC7682-7506

Greiner Bio One, \# 665180

Nunc, \# 167008

Perkin Elmer, Inc. \#1450-401

\section{1. 13 Buffers and solutions}

All buffers were prepared with deionized $\mathrm{H}_{2} \mathrm{O}$, if not indicated otherwise

Name

Plasmid dialysis

EB Buffer in Ampuwa water

TE Buffer in Ampuwa water $\underline{\text { Ingredients }}$

$\begin{array}{ll}\text { Tris- } \mathrm{HCl} & 10 \mathrm{mM} \\ \mathrm{pH} 8.5 & \\ \text { Tris-Cl } & 10 \mathrm{mM} \\ \text { EDTA } & 1 \mathrm{mM}\end{array}$

$\mathrm{pH} 8.0$ 
Transformation of component cells

5x KCM buffer

$\begin{array}{ll}\mathrm{CaCl}_{2} & 150 \mathrm{mM} \\ \mathrm{MgCl}_{2} & 250 \mathrm{mM} \\ \mathrm{KCl} & 500 \mathrm{mM}\end{array}$

LB Medium

LB medium powder $25 \mathrm{~g} / \mathrm{L}$

Ampicillin

$100 \mu \mathrm{g} / \mathrm{mL}$

LB plates

LB agar powder

$40 \mathrm{~g} / \mathrm{L}$

Ampicillin $100 \mu \mathrm{g} / \mathrm{mL}$

$\underline{\text { Transfection of HEK293A cells }}$

2x BBS

sterile filtrated

$\mathrm{Na}_{2} \mathrm{HPO}_{4}$

$1.5 \mathrm{mM}$

BES

$50 \mathrm{mM}$

$\mathrm{NaCl}$

$280 \mathrm{mM}$

$\mathrm{CaCl}_{2}$

$\mathrm{pH}$

$6.95(\mathrm{NaOH})$

$\mathrm{CaCl}_{2}$

$2.5 \mathrm{M}$

Adenovirus purification

10x Sucrose buffer

Tris

$24.22 \mathrm{~g}$

$\mathrm{MgCl}_{2} \times 6 \mathrm{H}_{2} \mathrm{O}$

$8.13 \mathrm{~g}$

$D(+)$ Sucrose

$800 \mathrm{~g}$

$\mathrm{pH}$

8

Aqua dest.

ad $2000 \mathrm{~mL}$

FRET measurements

FRET buffer

$\begin{array}{ll}\mathrm{NaCl} & 144 \mathrm{mM} \\ \mathrm{KCl} & 5.4 \mathrm{mM} \\ \mathrm{MgCl}_{2} & 1 \mathrm{mM} \\ \mathrm{CaCl}_{2} & 1 \mathrm{mM} \\ \mathrm{HEPES} & 10 \mathrm{mM} \\ \mathrm{pH} & 7.3\end{array}$




\section{2 Methods}

\section{2. 1 Generation of the recombinant plasmids and adenoviral construction of}

E1-camps and PLM-E1 biosensors

The development the cytosolic cAMP sensitive adenoviral biosensor was based on the already published DNA construct encoding for Epac1-camps ${ }^{249}$. This gene construct consists of a special nucleotide binding sequence (E157-E316) from the human Epac1 protein. We targeted the E1-camps biosensor to the PLM/NKA microdomain by using a special cloning strategy and thoroughly optimized the new PLM targeted FRET biosensor, which promised direct monitoring of cAMP dynamics in the vicinity of PLM. Following cloning strategies are used to produce reliable PLM-targeted biosensors and adenoviral constructs to monitor FRET changes in the vicinity of PLM (see sections 2. 2. 7-2. 2. 9).

\section{2. 2 PCR- Polymerase chain reaction}

To amplify DNA sequence of interest and introduce restriction sites to DNA sequences during cloning, following PCR reaction was applied.

$\begin{array}{ll}\mathrm{H}_{2} \mathrm{O} & 81 \mu \mathrm{l} \\ \text { 10x Pfu buffer } & 10 \mu \mathrm{l} \\ \text { dNTPs } 10 \mathrm{mM} & 2 \mu \mathrm{l} \\ \text { for. Primer }(25 \mu \mathrm{mol} / \mathrm{L}) & 2.5 \mu \mathrm{l} \\ \text { rev. Primer }(25 \mu \mathrm{mol} / \mathrm{L}) & 2.5 \mu \mathrm{l} \\ \text { DNA sequence }(200 \mathrm{ng}) & 1 \mu \mathrm{l} \\ \text { Pfu DNA Polymerase } & 1 \mu \mathrm{l}\end{array}$

Following PCR protocol was applied:

$\left(94^{\circ} \mathrm{C}\right.$ for $5 \mathrm{~min}, 30 \times\left(94^{\circ} \mathrm{C}\right.$ for $30 \mathrm{~s}, 55^{\circ} \mathrm{C}$ for $30 \mathrm{~s}, 72^{\circ} \mathrm{C}$ for $\left.\left.\mathrm{t}\right), 72^{\circ} \mathrm{C}-7 \mathrm{~min}\right)$ possible over night storage at $4{ }^{\circ} \mathrm{C}$.

The elongation time $(\mathrm{t})$ was dependent on the length of the desired PCR product and used enzyme (Pfu polymerase replicates $1 \mathrm{~kb}$ in $2 \mathrm{~min}$ ).

\section{2. 3 Gel electrophoresis, DNA purification and quantification}

A very important mean to analyze DNA sequences or to generate DNA segments for ligation and cloning, digestion with Type II restriction enzymes (New England Biolabs) was performed according to manufacturer's instructions. The available New England Biolabs buffer (NEBuffer) chart from the manufacturer simplified choosing the correct buffer for a digestion using a single or two enzymes. Following two protocols are applied in this project. 
control digestion of DNA for $0.5 \mathrm{~h}$ at $37^{\circ} \mathrm{C}$ :

$500 \mathrm{ng}$ template (or $8 \mu \mathrm{L}$ from a mini-preparation)

$1.5 \mu \mathrm{L}$ NEBuffer (suitable for both enzymes)

$\left.\begin{array}{l}0.25 \mu \mathrm{L} \text { restriction enzyme } 1 \\ 0.25 \mu \mathrm{L} \text { restriction enzyme } 2\end{array}\right\}$ or 0.5 of a single enzyme

ad $15 \mu \mathrm{L} \mathrm{H} 2 \mathrm{O}$

The DNA segments were run on a $1 \%$ agarose gel and analyzed quantitatively using the Multi Image Light Cabinet Transilluminator (Alpha Innotech Corporation).

segmental digestion of DNA for cloning:

6-8 $\mu \mathrm{g}$ of vector plasmid or template DNA

$5 \mu \mathrm{L}$ NEBuffer

$2.5 \mu \mathrm{L}$ of restriction enzyme 1

$2.5 \mu \mathrm{L}$ of restriction enzyme 2

ad $50 \mu \mathrm{L} \mathrm{H} 2 \mathrm{O}$

digestions were incubated over night at $37^{\circ} \mathrm{C}$

DNA was isolated by gel electrophoresis (2. 2. 4), extracted and eluted in $50 \mu \mathrm{L}$ EB-buffer (vector fragment) or $25 \mu \mathrm{L}$ EB-buffer (insert).

\section{2. 4 Gel electrophoresis, DNA purification and quantification}

Upon generating the PCR product or digestion with restriction enzymes, the DNA fragments were run on a $1 \%$ agarose gel (TAE buffer including $0.5 \mu \mathrm{g} / \mathrm{ml}$ ethidium bromide) at 100-150 V. 1000 bp ladder (Quickload) was run in parallel, as DNA marker. Documentation of the results were prepared as a picture and printed out from the system. The DNA molecules were separated according to their size, DNA segment of interest were excised with a scalpel and extracted and purified using the QIAquick Gel Extraction Kit according to the manufacturer's protocol. The DNA was eluted in TE-buffer (or EB-buffer after BP and LR reaction). The Nanodrop device was utilized to determine the DNA concentration.

\section{2. 5 Ligation}

Inserting DNA fragments into a vector plasmid with matching restriction ends requires the application of T4-DNA Ligase to connect the insert with the vector: 
Ligation mix:

T4 DNA Ligase

$1 \mu \mathrm{L}$

vector $(1 \mu \mathrm{g})$

$1 \mu \mathrm{L}$

digested insert(s)/or digested PCR product(s)

$11.5 \mu \mathrm{L}$

10x ligase reaction buffer

$1.5 \mu \mathrm{L}$

overnight incubation at $14^{\circ} \mathrm{C}$

For a triple ligation, the different molecular masses of the used inserts are crucial to calculate vector-to-insert ratios. In this case, two insert samples are used in $11.5 \mu \mathrm{l}$ in a ratio adjusted to their molecular weights to provide equimolar amounts.

\section{2. 6 Transformation of competent bacteria cells}

The generated plasmid DNA sequence was introduced into One Shot ${ }^{\circledR}$ TOP10 chemically competent $E$. coli or One Shot ${ }^{\circledR}$ OmniMax2T1 (for BP and LR reaction) incubating following reaction mix for $50-60 \mathrm{~min}$ at $37^{\circ} \mathrm{C}$.

$\begin{array}{ll}\text { E.coli competent cells } & 100 \mu \mathrm{L} \\ \text { Ligation Mix } & 15 \mu \mathrm{L} \\ \mathrm{H}_{2} \mathrm{O} & 65 \mu \mathrm{L} \\ 5 \times \mathrm{KCM} \text { Buffer } & 20 \mu \mathrm{L} \text { (see 2.1.13) }\end{array}$

The reaction mix was incubated on ice for $20 \mathrm{~min}$ followed by $10 \mathrm{~min}$ at RT. To simplify bacterial growth, $1 \mathrm{~mL}$ of LB medium (without antibiotics) was added to the mixture and incubated at $37^{\circ} \mathrm{C}$ in a ThermoMixer $(700 \mathrm{rpm})$, pelletized and resuspended in $100 \mu \mathrm{L}$ of the aspirated supernatant (LB medium without antibiotics). Thereafter, the suspension was spread on ampicillin selective LB- medium plates (kanamycin selective plates are used after $\mathrm{BP}$ reaction) and colonies were grown overnight at $37^{\circ} \mathrm{C}$ and harvested in order to be raised in $4 \mathrm{~mL}$ LB medium with ampicillin (kanamycin) overnight at $37^{\circ} \mathrm{C}$. The next day plasmid DNA was precipitated out of the bacteria using the Qiagen Plasmid Mini Kit for a $2 \mathrm{~mL}$ volume of bacterial suspension. Subsequent DNA restriction analysis demonstrated positive clones, which were used grown in $30 \mathrm{~mL}$ of LB medium with ampicillin over night at $37^{\circ} \mathrm{C}$ and plasmid DNA was extracted using the Qiagen Plasmid Midi Kit. The concentration of DNA was measured using the Nanodrop device, and plasmid DNA sequencing was performed at Eurofins Genomics. 


\section{2. 7 Cloning the human PLM-E1 FRET biosensor based on the pcDNA3 Epac1-camps construct}

Our aim was to target the cytosolic cAMP sensor to the PLM microdomain via fusing it to the C-terminus of a full length human PLM sequence via a flexible linker GSGSAS. For this, the Epac1-camps plasmid was double digested with HindIII and EcoRI restriction enzymes to remove the N-terminal YFP sequence. The following two inserts were amplified by PCR: 1.) Full human PLM sequence without stop codon was amplified using the primers PLMHindlllfor and PLMNhelrev; 2.) YFP sequence was amplified using YFPNhelfor and YFPEcoRIrev primers. The PCR products were run on an $1 \%$ agarose gel, purified and digested with HindlII/Nhel and Nhel/EcoRI, respectively, as described in 2 .2. 4. After DNA purification, both extracted and predigested DNAs of PLM and YFP were triple ligated with the HindIII/EcoRI digested pcDNA3-Epac1-camps vector sequence.

\section{2. 8 Cloning the canine PLM-E1 FRET biosensor based on the PLM-YFP construct}

After some initial experiments detailed in section 3. 1, an optimized sequence of the PLM-E1 biosensor was designed with the help of Dr. William Fuller, who kindly provided an alternative PLM-YFP sequence having a longer flexible linker between PLM and YFP. To retain this liner sequence KRSRAQASNSAVDGTPVATG, $6 \mu \mathrm{g}$ of the given PLM-YFP vector was double digested with $\mathrm{Kpnl}$ and Notl. Two inserts were prepared by: 1.) PCR amplification of the YFP sequence using the pEYFP-N1 vector as a template with the primers PLMYFPKpnlfor and YFPEcoRIrev as described in 2. 2. 2. The PCR product was purified and digested with $\mathrm{Kpnl}$ and EcoRI; 2.) $10 \mu \mathrm{g}$ of the Epac1-camps plasmid were digested with EcoRI and Notl to obtain the second fragment which includes Epac1-CFP sequence. Both digested fragments and the vector were run on a $1 \%$ gel and the DNA was extracted using the Qiaquick Gel Extraction Kit. To ligate the pEYFP N1 caninePLM-linker vector, eluted in 50 $\mu \mathrm{l}$ elution buffer, with the YFP and Epac1-CFP fragments, each eluted in $25 \mu$ l elution buffer, following mix is prepared for an overnight ligation at $14^{\circ} \mathrm{C}$ :

pEYFP N1 caninePLM-linker ( 4200 bp) $1 \mu \mathrm{l}$

YFP $(\sim 700 \mathrm{bp})$

Epac1-CFP ( 1200 bp) $5 \mu \mathrm{l}$

T4 Ligase buffer 10x $\quad 1.5 \mu \mathrm{l}$

T4 Ligase $1 \mu \mathrm{l}$ 


\section{2. 9 Generation of the adenoviral constructs Epac1-camps, humanPLM-E1 and PLM-E1}

The adenovirus generation is performed via the Gateway ${ }^{\circledR}$ Cloning system and in accordance with the manufacturer's instructions.

The sequence of interest embedded in the pcDNA3.0 or pEYFP-N1 vector was amplified by PCR using specially designed primers with flanking attB sequences.

Epac1-camps and ${ }_{\text {human }}$ PLM-E1

caninePLM-E1

$\begin{array}{llll}\mathrm{H}_{2} \mathrm{O} & 81 \mu \mathrm{l} & \mathrm{H} 2 \mathrm{O} & 81 \mu \mathrm{l} \\ \text { 10x Pfu buffer } & 10 \mu \mathrm{l} & \text { 10x Pfu buffer } & 10 \mu \mathrm{l} \\ \text { dNTPs } 10 \mathrm{mM} & 2 \mu \mathrm{l} & \text { dNTPs } 10 \mathrm{mM} & 2 \mu \mathrm{l} \\ \text { pcDNA3attBFor }(10 \mathrm{pmol} / \mu \mathrm{l}) & 2.5 \mu \mathrm{l} & \text { attBPLMhundfor }(10 \mathrm{pmol} / \mu \mathrm{l}) & 2.5 \mu \mathrm{l} \\ \text { pcDNA3attBRev }(10 \mathrm{pmol} / \mu \mathrm{l}) & 2.5 \mu \mathrm{l} & \text { attBYFPN1 rev }(10 \mathrm{pmol} / \mu \mathrm{l}) & 2.5 \mu \mathrm{l} \\ \text { pcDNA3 biosensor DNA }(200 \mathrm{ng}) & 1 \mu \mathrm{l} & \text { pEYFP N1 PLM-E1 }(200 \mathrm{ng}) & 1 \mu \mathrm{l} \\ \text { Pfu DNA Polymerase } & 1 \mu \mathrm{l} & \text { Pfu DNA Polymerase } & 1 \mu \mathrm{l}\end{array}$

The PCR reaction was as follows:

$94^{\circ} \mathrm{C} 5 \mathrm{~min}$

$94^{\circ} \mathrm{C} 30 \mathrm{sec}$

$55^{\circ} \mathrm{C} 30 \mathrm{sec}$

$72^{\circ} \mathrm{C} 4 \min 30 \mathrm{sec}(4 \mathrm{~min}$ for Epac1-camps)

$72^{\circ} \mathrm{C} 7 \mathrm{~min}$

PCR product were separated on a $1 \%$ agarose gel and purified; the DNA concentration was determined with the Nanodrop system. Each of the constructs were introduced into the donor vector Gateway ${ }^{\circledR}$ pDONR ${ }^{\mathrm{TM}} 221$ (attP) with in vitro recombination reaction (BP reaction) by means of the Gateway ${ }^{\circledR}$ BP Clonase ${ }^{\mathrm{TM}}$ II Enzyme Mix.

The cloned vector was transformed into One Shot ${ }^{\circledR} O m n i M a x 2 T 1$ bacteria and grown overnight at $37{ }^{\circ} \mathrm{C}$ on a kanamycin selective LB-medium plate. The grown colonies were selected and amplified, as described in 2. 2. 6. The control digestion was performed with EcoRI/EcoRV for Epac1-camps and humanPLM-E1 as well as with Kpnl/Notl for caninePLM-E1. To generate the virus expression vector containing the biosensor DNA construct, the BP reaction product was further recombined with $\mathrm{pAd} / \mathrm{CMV} / \mathrm{V} 5-\mathrm{DEST} \mathrm{T}^{\mathrm{TM}}$ through the LR-reaction overnight at $25^{\circ} \mathrm{C}$. The $L R$ product with the final recombinant adenoviral vector DNA was grown in One Shot ${ }^{\circledR}$ OmniMax2T1 cells and selected by using ampicillin instead of kanamycin LB-medium plates. The positive clone was identified by restriction enzymes EcoRI for Epac1-camps, Nhel for humanPLM-E1 and Xbal for caninePLM-E1. 
Following mixtures are prepared and incubated overnight at $25^{\circ} \mathrm{C}$.

\section{$B P$ reaction}

PCR eluate

pDONR $^{\text {TM }} 221$ vector

(150ng)

TE-buffer

BP Clonase II Enzyme mix

\section{$L R$ reaction}

DNA

$150 \mathrm{ng}$

$\mathrm{pAd} / \mathrm{CMV} / \mathrm{V} 5-\mathrm{DEST} \mathrm{TM}^{\mathrm{TM}} \quad 1 \mu \mathrm{L}$

(150ng)

ad $8 \mu \mathrm{L} \quad$ TE-buffer

ad $8 \mu \mathrm{L}$

$2 \mu \mathrm{L} \quad$ LR Clonase II Enzyme mix $2 \mu \mathrm{L}$

Before HEK293A transfection, Epac1-PLN adenoviral vector DNA was linearized as follows:

$\begin{array}{ll}\text { DNA } & 5 \mu \mathrm{g} \\ \text { 10x buffer NEB1 } & 2.5 \mu \mathrm{l} \\ \text { 100x BSA } & 0.25 \mu \mathrm{l} \\ \mathrm{Pacl} & 2 \mu \mathrm{l} \\ \mathrm{H}_{2} \mathrm{O} \text { ad } & 25 \mu \mathrm{l}\end{array}$

Extraction of the DNA is achieved through ethanol precipitation of the digested vector. To initiate the precipitation by electrophilic interaction between negatively charged phosphate groups of the DNA and positively charged sodium ions in solution, $10 \mu \mathrm{l}$ of $3 \mathrm{M}$ sodium acetate solution was added to the digestion mix and incubated for $30 \mathrm{~min}$ at $-20^{\circ} \mathrm{C}$. At the same time, $70 \mu$ l of ice-cold $100 \%$ ethanol was used as a less polar solvent than water to aid the functional groups to form stable ionic bonds and precipitate. After centrifugation for 10 min at $13.300 \mathrm{rpm}$, the generated DNA pellet was washed with $500 \mu \mathrm{l}$ ice-cold $75 \%$ ethanol and centrifuged again for $5 \mathrm{~min}$ at $13.000 \mathrm{rpm}$. In order to solubilize the DNA pellet, $10 \mu \mathrm{l}$ $\mathrm{H}_{2} \mathrm{O}$ was added. The solution was used for transfection of a $10 \mathrm{~cm}$ plate with HEK-293A cells at $80 \%$ confluency by the Lipofectamine ${ }^{\circledR} 2000$ reagent $(7 \mu \mathrm{L})$ in OPTI-MEM® $(300 \mu \mathrm{L})$, incubated for $20 \mathrm{~min}$ at RT. After 7-14 days, virus production led to detachment of transfected HEK293A cells. The detached cells with virus were harvested by centrifuging for $5 \mathrm{~min}$ at $800 \mathrm{rpm}$. The supernatant was used for further HEK293A transduction and virus amplification in Iscove Basal Medium (5\% FCS, $1 \%$ antibiotic, antimycotic). Performing a ultracentrifugation of the lysed virus containing supernatant of HEK293A cells (40.000 rpm overnight at $16{ }^{\circ} \mathrm{C}$, Beckmann Rotor Ti90). The virus was concentrated in a cesium chloride gradient during centrifugation. The developed virus band was collected using a 21 gauge needle and dialyzed in $10 \times$ sucrose buffer. To determine physical virus concentration, the optical density (OD260) was measured. After diluting the virus solution 1:100 with sucrose buffer containing $10 \%$ of sterile glycerol, the aliquots were labeled and stored at $-80^{\circ} \mathrm{C}$. Using 
a Plaque Assay (Cooper, 1961) the biological virus activity (plaque forming units per $\mathrm{ml}$ $[\mathrm{pfu} / \mathrm{mL}])$ was assessed. For this purpose, 6 -well plates of HEK293A cells covered with $1.5 \%$ plaque GP agarose were prepared and transduced with virus at 10, 100 and 1000 pfu/well (calculated via physical virus concentration) and analyzed. Adult rat ventricular cardiomyocytes were isolated as described in 2. 2. 19 and transduced with the E1camps/E1-PLM virus (MOI 300). Imaging experiments were performed $48 \mathrm{~h}$ after transduction.

\section{2. 10 Calculating the multiplicity of infection and adenoviral transduction of ARVM}

ARVMs were isolated as described (see section 2. 2. 19), counted in a Naubauer Chamber (Celeromics), seeded on 6-well plates and maintained in culture for 1-2 h. Approximately 11.5 million ARVMs in total were used for transduction. In order to calculate the volume of virus to achieve the desired multiplicity of infection (MOI) for a specific amount of cells, we used the following equation:

Adenovirus volume added $(\mu \mathrm{l})=($ Required $\mathrm{MOI} \times$ number of cells $) /(\mathrm{PFU} / \mu \mathrm{l})$

The calculated amount was added to a volume of fresh culture medium necessary to cover the cells to be transduced ( $2 \mathrm{~mL} /$ well). The culture medium (Medium 199 supplemented with $5 \mathrm{mmol} / \mathrm{L}$ taurine, $5 \mathrm{mmol} / \mathrm{L}$ carnitine, $5 \mathrm{mmol} / \mathrm{L}$ creatine, penicillin/streptomycin and Lglutamine supplement) of ARVMs was replaced with the viral culture medium, and the ARVMs were further incubated with the adenovirus for $48 \mathrm{~h}$ at $37^{\circ} \mathrm{C}$ and $5 \% \mathrm{CO}_{2}$. In case of relatively high amount of dead ARVMs, the culture medium was replaced after $24 \mathrm{~h}$.

\section{2. 11 Cell culture and transfection of the HEK293A cell line}

In order to test newly developed FRET biosensors, HEK293A cells were cultured at $37^{\circ} \mathrm{C}$ and $5 \% \mathrm{CO}_{2}$ using Dulbecco's Modified Eagle's Medium (DMEM medium) supplemented with $4.5 \mathrm{~g} / \mathrm{L}$ glucose, 10\% FCS, $2 \mathrm{mM} \mathrm{L}$-glutamine, $100 \mathrm{U} / \mathrm{mL}$ penicillin and $100 \mu \mathrm{g} / \mathrm{ml}$ streptomycin. For passaging, cells were washed with sterile phosphate buffered saline (PBS), detached from the surface of the wells/flask using $2 \mathrm{~mL} 0.05 \%$ Trypsin (Invitrogen), resuspended and split in a ratio of 1:10 in fresh cell medium.

Plated on coverslips in six well plates the HEK293A cells were ready for transfection in $24 \mathrm{~h}$, when they reached 60-70 \% confluency. In general, two different transfection methods were used, depending on the purpose of transfection: 1.) The $\mathrm{CaCl}_{2}$ method for having a reasonable expression rate to analyze the quality of the plasmid and 2.) the Lipofectamine 
method to accomplish enhanced transfection efficiency for protein overexpression and quantification analysis.

\section{2. 11. 1 Transfection with $\mathrm{CaCl}_{2}$ under sterile conditions:}

$10 \mu \mathrm{l}$ of Plasmid DNA $(1 \mu \mathrm{g} / \mu \mathrm{l})$ were added to $440 \mu \mathrm{l}$ of sterile $\mathrm{H}_{2} \mathrm{O}$. The DNA dilution was mixed with $50 \mu \mathrm{l}$ of $2.5 \mathrm{M} \mathrm{CaCl}_{2}$ and $500 \mu \mathrm{l}$ 2x BBS. After 10 min incubation at RT, $166 \mu \mathrm{l}$ of the mixture were added dropwise to each well.

\section{2. 11. 2 Transfection with Lipofectamine $2000 \AA$ under sterile conditions:}

Following the manufacturer's instructions for optimal transfection, $36 \mu \mathrm{l}$ of the Lipofectamine $2000 \AA$ reagent were diluted with $846 \mu$ l Opti-Mem ${ }^{\circledR}$ Medium, and $1.2 \mu \mathrm{l}$ of the DNA Plasmid $(1 \mu \mathrm{g} / \mu \mathrm{l})$ in $150 \mu \mathrm{l}$ of the same medium. After mixing both dilutions, a DNA-lipid complex was allowed to develop for $20 \mathrm{~min}$. Thereafter, $250 \mu \mathrm{l}$ of the reaction mix was dropwise pipetted onto each well with HEK293 cells in culture medium and the experiments were conducted after a $24 \mathrm{~h}$ incubation at $37^{\circ} \mathrm{C}$ and $5 \% \mathrm{CO}_{2}$.

To transfect cells with newly generated viral vectors (see section 2. 2. 9), the transfection mix contained $300 \mu \mathrm{L}$ Opti-MEM, $5 \mu \mathrm{g}$ linearized DNA in the pAd_CMV_V5DEST vector and $7 \mu \mathrm{L}$ Lipofectamine $2000 \AA$ reagent for a $10 \mathrm{~cm}$ plate of HEK293A cells.

\section{2. 12 SDS-PAGE and Western Blot analysis}

Using SDS-PAGE, proteins were separated according to their size by sodium dodecyl sulfate polyacrylamide gel electrophoresis, whereas in Western Blotting, separated proteins were transferred from the gel onto a polyvinylidene fluoride or nitrocellulose membrane and analyzed for protein expression by using specific antibodies.

\section{Experimental methods for the project phase in London:}

For expression analysis of the humanPLM-E1 sensor to be examined, transduced HEK293 cells were lysed in $500 \mu \mathrm{L} 2 \times$ SDS sample buffer (100 mMol Tris, 4\% SDS, 20\% glycerol, 0.2\% bromphenol blue, $5 \%$-mercaptoethanol in $\mathrm{H}_{2} \mathrm{O}, \mathrm{pH}$ 6.8) per well (if not indicated otherwise), scraped and harvested into $1.5 \mathrm{~mL}$ tubes. Gels were prepared beforehand according to following instructions, starting with a 10\% acrylamide (Protogel, National Diagnostics) resolving gel, suitable for a $75 \mathrm{kD}$ large protein. 
Table 4. Ingredients of the used gels for Western Blot analysis at King's College London

\begin{tabular}{|c|c|c|}
\hline Ingredient & Resolving gel (10\%) & Stacking gel (4.5\%) \\
\hline Tris $\mathrm{HCl}(1 \mathrm{M}) \mathrm{pH} 8.8$ & $3.75 \mathrm{~mL}$ & - \\
\hline Tris $\mathrm{HCl}(1 \mathrm{M}) \mathrm{pH} 6.8$ & - & $0.625 \mathrm{~mL}$ \\
\hline Acrylamide (30\%) & $3.33 \mathrm{~mL}$ & $0.75 \mathrm{~mL}$ \\
\hline SDS (10\%) & $0.1 \mathrm{~mL}$ & $50 \mu \mathrm{L}$ \\
\hline TEMED & $5 \mu \mathrm{L}$ & $10 \mu \mathrm{L}$ \\
\hline APS (10\%) & $50 \mu \mathrm{L}$ & $80 \mu \mathrm{L}$ \\
\hline $\mathrm{H}_{2} \mathrm{O}$ & $2.76 \mathrm{~mL}$ & $3.465 \mathrm{~mL}$ \\
\hline
\end{tabular}

Protein samples and protein ladder (Precision Plus Protein TM Dual-Colour Standards [BioRad]) were run at constant $100 \mathrm{~V}$ for about 1.5 hours in SDS running buffer (0.025 M TRIS, $0.19 \mathrm{M}$ glycine, $1 \% \mathrm{SDS}$ ). The protein migration continued until the blue dye front reached the bottom of the resolving gel. The SDS-PAGE was performed using the Mini protean III Tetra Cell system (Bio-Rad). Upon separating the proteins according to size in the resolving gel, they were transformed onto polyvinylidene fluoride (PVDF) membranes (GE Healthcare) prerinsed with methanol and transfer buffer (0.025 M TRIS, $0.19 \mathrm{M}$ glycine, $20 \%$ methanol, $0.1 \%$ SDS). The gels on membranes were sandwiched between six layers of filter paper presoaked in transfer buffer. Next, the transfer in a Hoeffer TE77 semi-dry transfer unit was carried out at $10 \mathrm{~V}$ for $35 \mathrm{~min}$ with a current restricted to $250 \mathrm{~mA}$ per gel.

The proteins were blocked against non-specific binding with PBST (PBS + $0.1 \%$ Tween) + $5 \%$ milk powder (Marvel) over night at $4^{\circ} \mathrm{C}$, the membrane was then treated with relevant primary antibodies (diluted in blocking buffer) for $2 \mathrm{~h}$ at room temperature (RT). The specific dilutions for primary antibodies are detailed in Table 1. The membranes were washed for one hour (each washing step: 10-15 min). To visualize the resulting primary antibody binding, the probes were incubated with horseradish peroxidise (HRP)-conjugated secondary antibody (diluted in blocking buffer) for $2 \mathrm{~h}$ at RT. The specific dilutions for secondary antibodies incubations are detailed in Table 2. After another washing procedure for one hour, the membranes were ready for enhanced chemiluminescence (ECL) analysis. Here, the HRP conjugated antibodies formed a complex with the ECL reagent mixture, detected via Hyperfilm ECL high performance X-ray film (GE Healthcare) for different acquisition times and developed using a Fuji RGIl automatic processor (Fuji). Upon scanning the protein bands, they were subjected to densiometric analysis. 


\section{Experimental methods for the project phase in Göttingen:}

Having produced the caninePLM-E1 FRET biosensor construct, SDS-PAGE/Western Blot analysis for characterization of expression and localization of the optimized sensor was performed. Apart from the following small modification, it was accomplished as at King's College London.

Cells (HEK293A or ARVMs) to be analyzed were lysed by $500 \mu \mathrm{L} /$ well $3 \times$ SDS-sample buffer (200 mM Tris, 6 \% SDS, $15 \%$ glycerol, $0.3 \%$ bromphenol blue, $10 \%$ 2-mercaptoethanol in $\mathrm{H}_{2} \mathrm{O}, \mathrm{pH} 6.7$ ) and harvested. Next, 2 gels for SDS-PAGE analysis were prepared as follows.

Table 5. Ingredients of the used gels for Western Blot analysis in UMG, Göttingen

\begin{tabular}{|c|c|c|c|}
\hline Ingredient & Resolving gel (10\%) & Resolving gel (12\%) & Stacking gel (4.5\%) \\
\hline $\begin{array}{l}\text { 4x Tris/SDS pH 8.8 (500 } \\
\mathrm{mmol} / \mathrm{L} \text { Tris, } 0.4 \% \text { SDS) }\end{array}$ & $3.0 \mathrm{~mL}$ & $3.0 \mathrm{~mL}$ & - \\
\hline $\begin{array}{l}4 \times \text { Tris/SDS pH 6.8 (500 } \\
\mathrm{mmol} / \mathrm{L} \text { Tris, } 0.4 \% \text { SDS) }\end{array}$ & - & - & $940 \mu \mathrm{L}$ \\
\hline Acrylamide $(30 \%)$ & $4 \mathrm{~mL}$ & $4.8 \mathrm{~mL}$ & $18.8 \mu \mathrm{L}$ \\
\hline TEMED & $18 \mu \mathrm{L}$ & $18 \mu \mathrm{L}$ & $7.5 \mu \mathrm{L}$ \\
\hline APS $(10 \%)$ & $48 \mu \mathrm{L}$ & $48 \mu \mathrm{L}$ & $80 \mu \mathrm{L}$ \\
\hline $\mathrm{H}_{2} \mathrm{O}$ & $5 \mathrm{~mL}$ & $4.2 \mathrm{~mL}$ & $2.31 \mathrm{~mL}$ \\
\hline
\end{tabular}

After running the proteins on a gel, they were transferred onto nitrocellulose membranes (Protran, Whatman) inside the Mini Trans-Blot Transfer Cell (Bio-Rad), filled up with ice cold transfer buffer (32 mM Tris, $192 \mathrm{mM}$ glycine, $4 \%$ methanol) and blotted for $2 \mathrm{~h}$ at $100 \mathrm{~V}$. To precisely cut the membrane in order to detect multiple proteins for one probe, it was stained with Ponceau S solution ( $0.5 \%$ in $10 \%$ acetic acid) for 5 min, washed with water until bands appeared. As described above, the membrane had to be blocked against unspecific binding with $5 \%$ milk in TBST (TBS $+1 \%$ Tween), this time at RT for $1 \mathrm{~h}$. The incubation with primary antibodies (for dilutions see section 2. 1. 9) was done at $4^{\circ} \mathrm{C}$ overnight. After membrane incubation with a HRP-coupled secondary antibody (see section 2.1.9) for an hour at RT and treatment with ECL (Thermo Scientific). Films (Fuji Medical X-Ray Films, Fujifilm) were developed using a film processor (SRX 101A Konica, USA) with varying exposure times (1 $\mathrm{s}-10 \mathrm{~min})$, scanned and analyzed. 


\section{2. 13 Co-immunoprecipitation}

With this co-immunoprecipitation (co-IP) protocol we were able to prove physical association of proteins expressed either in HEK293A cells or ARVMs. All samples are handled at $4^{\circ} \mathrm{C}$ during the entire procedure. The lysed protein amount used for one co-IP experiment was equal to cells from 1-3 wells of one six well plate (1 well for transfected HEK293A cells and 3 wells for transduced ARVMs). $20-30 \mu \mathrm{L}$ of the GFP-Trap $\AA$ reagent (Chromotek) was washed $5 \times 1 \mathrm{~mL}$ in lysis buffer containing $2 \mathrm{mg} / \mathrm{mL}$ octaethylene glycol monododecyl ester (C12E10) in co-IP buffer (1 $\mathrm{mM}$ EDTA in PBS plus protease and phosphates inhibitors) and equilibrated overnight at $4^{\circ} \mathrm{C}$. Upon lysing the cells in $500 \mu \mathrm{L}$ co-IP lysis buffer, the cells were carefully scraped from the bottom of the well, agitated at $4^{\circ} \mathrm{C}$ and centrifuged $(17000 \mathrm{~g})$ for $5 \mathrm{~min}$ at $4^{\circ} \mathrm{C}$. The supernatant of the lysed centrifuged cells was incubated with the GFP-Trap $\AA$ beads over night at $4^{\circ} \mathrm{C}$. Thereafter, the beads were washed 5 times $\left(1 \mathrm{~mL}\right.$ each) with $4^{\circ} \mathrm{C}$ co-IP buffer $(0.5 \mathrm{mg} / \mathrm{mL}$ C12E10). The beads were then resuspended in $100 \mu \mathrm{L} 2 \mathrm{x}$ SDS PAGE sample buffer supplemented with $5 \% \beta$-mercaptoethanol.

The same protocol was applied for co-IP analysis by means of primary antibody immobilized on Protein G Sepharose 4 Fast Flow ${ }^{\circledR}$ (GE Healthcare). $20 \mu \mathrm{L}$ sepharose beads were washed with co-IP lysis buffer $\left(5 \times 1 \mathrm{~mL}\right.$ ) and equilibrated overnight at $4^{\circ} \mathrm{C}$ (or for $1-2 \mathrm{~h}$ at $\mathrm{RT}) .1 \mu \mathrm{g}$ of primary antibody was pre-immobilized on the beads, again over night at $4^{\circ} \mathrm{C}$. The beads were incubated with solubilized and centrifuged supernatants of cells (HEK293A or ARVM) from 1-3 wells of a six-well plate overnight at $4^{\circ} \mathrm{C}$.

Collecting samples at each step throughout the entire procedure, including the multiple washing steps, was important to trail protein loss and to facilitate the comparative analysis of starting, unbound and IP - fractions.

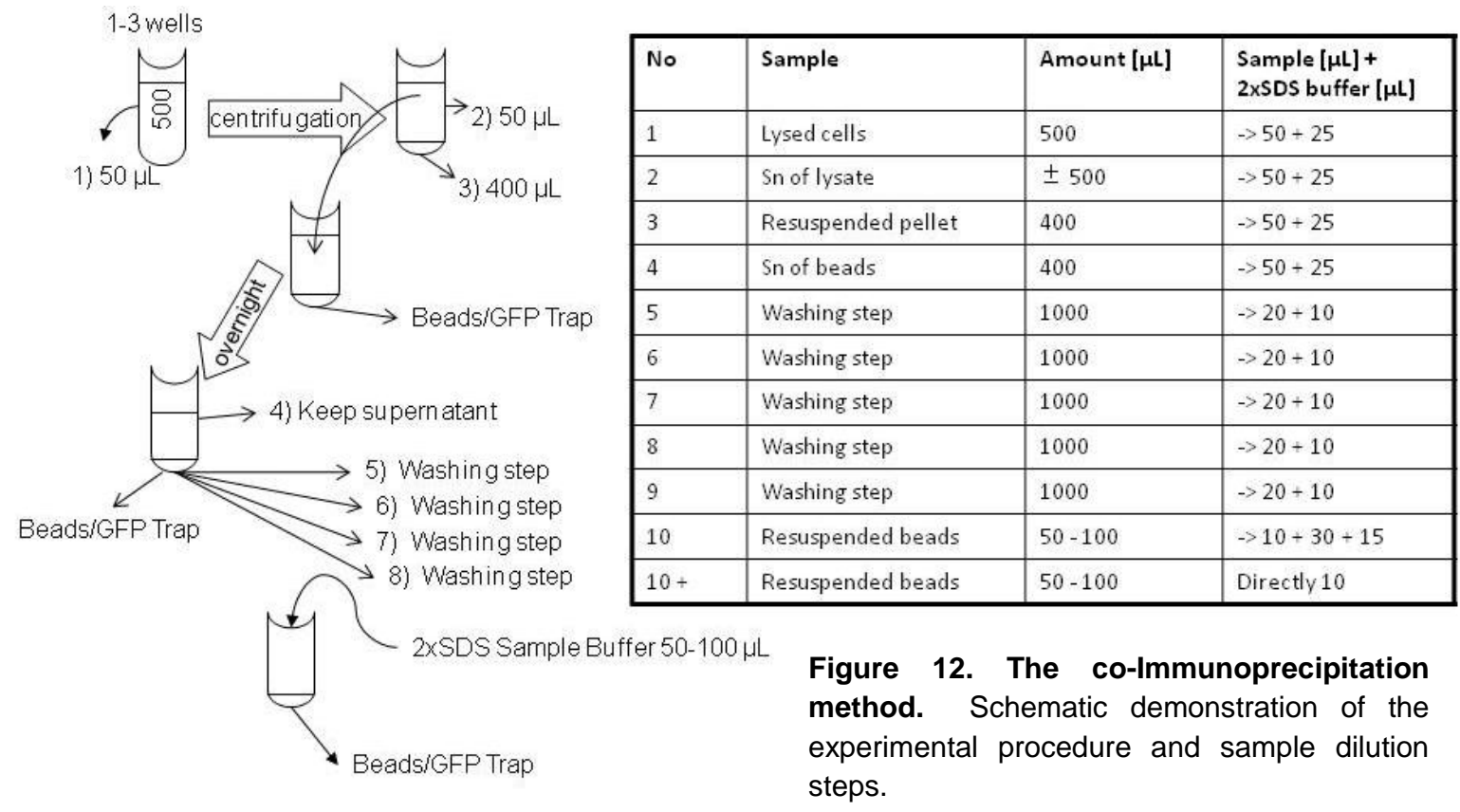




\section{2. 14 Cell surface biotinylation}

Testing the cell surface expression of the PLM-E1 DNA construct was essential to prove correct localization and expression of the developed tagged FRET biosensor. A significant amount of biosensor protein was expected to reach the cell surface. To verify this, HEK293 cells were seeded and cultivated as mentioned in section and transfected with the PLM-E1 plasmid at a confluency of $65 \%$. One well of a six well plate was washed three times very slowly and carefully with $37^{\circ} \mathrm{C}$ PBS. Biotinylation solution $(1 \mathrm{mg} / \mathrm{mL}$ sulfo-SS-NHS-biotin in PBS) was added to the cells and incubated for $10 \mathrm{~min}$ at $37^{\circ} \mathrm{C}$ in incubator. After rinsing the cells to remove the excess biotinylation reagent, cells were lysed in $0.5 \mathrm{~mL}$ per well of $1 \%$ triton X-100 in PBS supplemented with protease inhibitors (lysis buffer) and lysed for 30 min at $4^{\circ} \mathrm{C}$. Samples were transferred to tubes (scraped and harvested from plate surface) and spun down at $17500 \mathrm{G}$ for $5 \mathrm{~min}$ at $4^{\circ} \mathrm{C}$. The supernatant was kept for further analysis, additionally one sample $(50 \mu \mathrm{L})$ was retained from it and mixed 1:1 with $2 x$ SDS PAGE sample buffer without $\beta$-mercaptoethanol (refer to as starting material). The retained supernatant was added to Streptavidin-Sepharose beads. Beforehand $\sim 30 \mu \mathrm{L}$ beads per sample were washed multiple times, preequilibrated in lysis buffer at $4^{\circ} \mathrm{C}$ and recovered by centrifuging $1 \mathrm{~min}$ at $17500 \mathrm{G} 4^{\circ} \mathrm{C}$. The beads were incubated with the supernatant of the lysed cell pellet for $1-4$ hours at $4^{\circ} \mathrm{C}$ or overnight. The beads were spun down at $17500 \mathrm{G}$ for 2 min at $4^{\circ} \mathrm{C}$. A small sample $(100 \mu \mathrm{L})$ was taken and mixed 1:1 with $2 x$ SDS-PAGE sample buffer with $5 \% \beta$ - mercaptoethanol (refer to as unbound fraction). The beads were washed with lysis buffer. Following final washing step, $100 \mu \mathrm{L} 2 x$ SDS-PAGE sample buffer with $5 \%$ $\beta$-mercaptoethanol was added. Before SDS-PAGE/Western Blot analysis, samples were heated at $60^{\circ} \mathrm{C}$ for $15 \mathrm{~min}$ to elute proteins in the bound fraction (refer to as beads fraction).

\section{2. 15 Generation of a HEK293A cell line stably expressing PLM-E1}

Using the principle of selection via antibiotic resistance, HEK293A cells exclusively expressing the PLM-E1 construct were selected, propagated and stocked at $-80^{\circ} \mathrm{C}$.

HEK293A cells were seeded on a $10 \mathrm{~cm}$ dish to achieve around $75 \%$ confluency overnight. On the next day, they were transfected with a mix of $300 \mu \mathrm{g}$ DMEM Medium without supplements, $3 \mu \mathrm{g}$ PLM-E1 DNA plasmid and $7 \mu \mathrm{L}$ Lipofectamine 2000®. One day after transfection, the medium was changed to DMEM medium containing $4 \mu \mathrm{L} / \mathrm{mL}$ Geniticin antibiotic $(50 \mathrm{mg} / \mathrm{mL}$, Thermo Fischer). Non-transfected cells usually started to detach during after one week of incubation at $37^{\circ} \mathrm{C}$, while transfected cells started to form small colonies, which were detectable macroscopically and could be picked. Using a flexible tube and a pasteur pipette, the colonies were picked by sucking and blowing each of the colonies back into wells of a 12-well plate containing DMEM Medium without supplements ( $1 \mathrm{~mL} /$ well). After 
overnight incubation at $37^{\circ} \mathrm{C}$, cells were treated again with Genticin, which permitted PLM-E1 expressing HEK293A cells to proliferate and to reach maximum confluency. Next, cells were transferred to a 6-well plate, and subsequently to a $10 \mathrm{~cm}$ dish to propagate the line. After sufficient amount of cells has been cultured, they were washed with PBS, trypsinized and resuspended in the $10 \mathrm{~cm}$ dish with $6 \mathrm{~mL}$ of DMEM culture medium without supplements, 1.7 $\mathrm{mL}$ FCS and $0.7 \mathrm{~mL}$ DMSO. The cell suspension was devided into Kryocups (1 mL), labelled and frozen at $-80^{\circ} \mathrm{C}$.

\section{2. 16 Ouabain sensitive ${ }^{86} \mathrm{Rb}$ uptake measurements}

We measured the ouabain sensitive ${ }^{86} \mathrm{Rb}$ uptake as a surrogate for potassium to determine the NKA activity of transfected HEK293A cells or untransfected cells as described by Howie et al. $^{252}$. Cells were cultured on 12 -well plates until they reached $70 \%$ confluency. The measurements were performed in presence of the sodium ionophore monensin (Sigma) to ensure consistent intracellular $\mathrm{Na}^{+}$and the $\mathrm{Na} / \mathrm{K} / 2 \mathrm{Cl}$ cotransporter inhibitor bumetanide (Sigma) to depress background uptake of ${ }^{86} \mathrm{Rb}$. Cells were rinsed with the extracellular solution and equilibrated with exactly $1 \mathrm{~mL} /$ well of same solution for $20 \mathrm{~min}$ at $37^{\circ} \mathrm{C}$. During change of the medium, we added $100 \mu \mathrm{M}$ ouabain to half of the wells and PBS (vehicle) to the other half and incubated at $37^{\circ} \mathrm{C}$ for 5 min before initiating ${ }^{86} \mathrm{Rb}$ uptake by the addition of $1 \mu \mathrm{Ci} / \mathrm{mL}{ }^{86} \mathrm{Rb}$ per well. The cells were incubated at $37^{\circ} \mathrm{C}$ for exactly $15 \mathrm{~min}$ previous to stopping the uptake by rapidly washing steps in three baths of ice cold PBS. The complete wash protocol should take $\sim 30 \mathrm{sec}$. Hereupon, cells were lysed in $0.2 \mathrm{~mL}$ lysis buffer per well (1\% Triton-100 in PBS). The specific activity of the ${ }^{86} \mathrm{Rb}$ solution is determined through $3 x$ measurements of $1 \mu \mathrm{L}{ }^{86} \mathrm{Rb}$ solution in 3 scintillation vials ('total counts vials'). Next to this, $3 x$ $50 \mu \mathrm{L}$ of each cell lysate per well was transferred to 3 separate scintillation vials for. Likewise $3 \times 5 \mu \mathrm{L}$ cell lysate volume to $3 x$ cuvettes containing protein assay reagent. The number of ${ }^{86} \mathrm{Rb}$ molecules of the lysates were measured in a liquid scintillation counter, and the protein content of lysates was measured in a spectrophotometer using a Bradford assay with bovine serum albumin as a standard.

\section{Extracellular solution (pH7.4)}

$20 \mathrm{~mm} \mathrm{NaCl}$

120mM NMDG-Cl

$1 \mathrm{mM} \mathrm{MgCl} 2$

$1 \mathrm{mM} \mathrm{CaCl} 2$

$5 \mathrm{mM} \mathrm{KCl}$
10mM HEPES

$10 \mu \mathrm{g} / \mathrm{mL}$ monensin

$10 \mathrm{mM}$ glucose

$100 \mu \mathrm{M}$ bumetanide 


\section{2. 17 Immunofluorescence}

Immunofluorescence antibody staining was performed on transduced and nontransduced cardiac myocytes to visualize expression, localization and co-localization of single and multiple stained proteins. Even though crosslinking agents, like paraformaldehyde, are better in preserving cell structure, they reduced the antigenicity of some cell components, which was the case in most of our test experiments. For this reason, cells were fixed for 20 min with ice-cold $99.9 \%$ ethanol at $-20^{\circ} \mathrm{C}$ to immobilize antigens. Fixed cells were either stored in PBS at $4^{\circ} \mathrm{C}$ or directly blocked with blocking buffer (20\% FCS and $0.15 \%$ Triton X in PBS) for $2 \mathrm{~h}$. The cells were washed with PBS and co-stained with rabbit polyclonal anti- FXYD1 (abcam) and either mouse monoclonal anti- $\alpha_{1}$ antibody (Millipore) or goat polyclonal antiPDE3A (Santa Cruz) antibodies in in blocking buffer over night at $4^{\circ} \mathrm{C}$, washed once with PBS, followed by the incubation with secondary antibodies diluted as mentioned in section 2 . 1. 9 for $2 \mathrm{~h}$ at RT. Confocal imaging was performed using Zeiss LSM 710 laser scanning confocal microscope (Carl Zeiss Microlmaging) equipped with a Plan-Apochromat x63/1.40 oil-immersion objective.

\section{2. 18 Heart failure rat model: Myocardial Infarction}

For the animal model used in this project, adult male Sprague Dawley rats (250-300 g) were subjected to proximal coronary ligation to induce chronic myocardial infarction as described before $^{145}$. All surgeries and experiments with failing cells were performed at Imperial College London (lab of Prof. Julia Gorelik). Briefly, the rats were anesthetized after treatment with antibiotics and buprenorphine $(0.03 \mathrm{mg} / \mathrm{kg})$. After an incision into the pleural space of the chest, the left anterior descending coronary artery was ligated by a suture with 6-0 silk. Sixteen weeks post surgery the Ml-rats were subjected to echocardiography analysis via Vevo, 770 micro-imaging system, Visualsonics. Upon extraction at 16 weeks post MI, hearts and aged matched control (AMC) hearts were weighed. The measured heart weight to tibia length ratio $(\mathrm{HW} / \mathrm{TL})$ served as an indicator of progressive hypertrophy next to ejection fraction volume, which was determined from M-mode of the used imaging system.

\section{2. 19 ARVM Isolation by Langendorff perfusion}

The adult rat ventricular myocytes were isolated by Langendorff perfusion by Tobias Goldak, (AG Nikolaev, Gö).

Before the isolation process started, the heart perfusion system was turned on in order to warm up the waterbath and its plastic tubing system. At $37^{\circ} \mathrm{C}$ reached in the water bath optimal tissue digestion could be performed. To do this, adult rats were anaesthetized and 
sacrificed by cervical dislocation. The thorax was cut open, leaving the atrias easy to access. $1 \mathrm{~mL}$ of Heparin (25000 I.E./5ml, Rotexmedica) was injected to one of the atrias. After reducing the bleeding with some paper tissue, the heart was rapidly excised. After washing the heart gently but quickly in $5 \mathrm{~mL}$ ice cold $1 \times$ perfusion buffer, it was transferred to a petri dish filled with ice cold $1 \times$ perfusion buffer. The aorta was mounted onto a grooved cannula with the help of two tweezers under a stereomicroscope as quickly as possible (not more than $2 \mathrm{~min}$ ), secured with a clip and tied with suture. The heart was perfused with $1 \mathrm{x}$ perfusion buffer through the tubing systems $(8 \mathrm{~mL} / \mathrm{min})$. As soon as the blood was washed (as judged by checking the color of the collected perfusion buffer, which went through the coronary arteries (1-2 min perfusion)), the solution was switched to the digestion buffer. We digested for 11 minutes, whilst collecting the collagenase buffer and recirculating by topping up the reservoir until the required time of the digestion is attained. Before the digestion time ended, $5 \mathrm{~mL}$ of digestion buffer was collected and transferred to a beaker. After 11 minutes of digestion the perfusion system was shut down and the ventricles of the heart were removed from the cannula into the prepared beaker. The ventricles were cut into small pieces to increase the surface area for digestion. The smaller pieces were homogenised via a $10 \mathrm{~mL}$ pipette mechanically. This procedure was repeated after adding $10 \mathrm{~mL}$ of stopping buffer into the cell suspension. The suspension was topped up to $25 \mathrm{~mL}$ with stopping buffer and filtered using a nylon mesh and left to settle for 8 min and washed twice with $25 \mathrm{~mL}$ stopping buffer having 8 min settlement time in between each step.

The sedimented cardiomyocytes were further subjected to re-calcification protocol. Intracellular calcium was increased in 3 steps to have a final concentration of $1 \mathrm{mM}$, letting the cells settle for 8 minutes after each step:

$\begin{array}{llll}25 \mathrm{~mL} \text { stopping buffer } & 2.5 \mu \mathrm{L} \mathrm{CaCl}_{2} 1 \mathrm{M} & \text { final concentration } & 100 \mu \mathrm{M} \\ 25 \mathrm{~mL} \text { of stopping buffer } & 10 \mu \mathrm{L} \mathrm{CaCl}_{2} 1 \mathrm{M} & \text { final concentration } & 400 \mu \mathrm{M} \\ 25 \mathrm{~mL} \text { of stopping buffer } & 22.5 \mu \mathrm{L} \mathrm{CaCl}_{2} 1 \mathrm{M} & \text { final concentration } & 900 \mu \mathrm{M}\end{array}$

Upon recalcification the cardiomyocytes were resuspended in $10 \mathrm{~mL}$ M199 medium with supplements. Before plating the freshly isolated ARVMs on laminin (Sigma) coated glass coverslips, the cells were counted in a counting chamber (Naubauer Chamber) in order to calculate the desired amount of cells per well for adenoviral transfection. Cells were maintained in culture at $37^{\circ} \mathrm{C} / 5 \% \mathrm{CO}_{2}$ for at least $2 \mathrm{~h}$ prior to use in biochemical/ adenoviral infection experiments. 
10 x Perfusion buffer (substances in mmol/L): $120.4 \mathrm{NaCL}, 14.7 \mathrm{KCl}, 0.6 \mathrm{KH}_{2} \mathrm{PO}_{4}, 0.6$ $\mathrm{Na}_{2} \mathrm{HPO}_{4} \times 2 \mathrm{H}_{2} \mathrm{O}, 1.2 \mathrm{MgSO}_{4} \times 7 \mathrm{H}_{2} \mathrm{O}, 10 \mathrm{Na}-\mathrm{HEPES}, \mathrm{pH} 7.4$ in $\mathrm{H}_{2} \mathrm{O}$.

1 x Perfusion buffer (substances in mmol/L): $4.6 \mathrm{NaHCO}_{3}, 30$ taurine, 10 2,3butanedione-monoxime, 5.5 glucose, $50 \mathrm{~mL} 1$ x perfusion buffer ad $500 \mathrm{~mL} \mathrm{H}_{2} \mathrm{O}$.

digestion buffer: straight before use, $50 \mathrm{~mL}$ of the perfusion buffer were supplemented with $40 \mu \mathrm{M} \mathrm{CaCl}_{2}$ and $0.1 \mathrm{~g}$ Collagenase Typ II.

stopping buffer: $130 \mathrm{~mL}$ supplemented with $10 \% \mathrm{FCS}$ and $12.5 \mu \mathrm{M} \mathrm{CaCl}_{2}$.

culture medium: $500 \mathrm{~mL}$ M199 medium (Sigma) was supplemented with $312.5 \mathrm{mg}$ taurine, $500 \mathrm{mg} \mathrm{D}, \mathrm{L}-$-Carnitine and $327.5 \mathrm{mg} \mathrm{D,L-Creatine,} 5 \mathrm{~mL}$ antibiotics (100 U/mL penicillin, 100 $\mu \mathrm{g} / \mathrm{mL}$ streptomycin).

Hearts from adult male rats with the induced myocardial infarction model and aged matched control rats were isolated as previously described ${ }^{253}$ by Peter $\mathrm{O}^{\prime}$ Gara at the Imperial College London.

\section{2. 20 FRET measurements of ARVMs transduced with PLM-E1 and E1-camps}

Cardiomyocytes on laminin coated coverslips underwent a 48 hours incubation upon adenovirus transduction and were washed off excessive viral particles once with fresh culture medium before starting the FRET experiments. The coverslips with transduced cells on them were mounted in the cell chamber, which was used during imaging experiments. Cells were washed once and $400 \mu \mathrm{L}$ fresh FRET buffer was added (300 $\mu \mathrm{L}$ if one was using 3 or more substances during experiments). All substances used during FRET experiments were diluted in FRET buffer. Using a drop of immersion oil, the chamber was placed onto the objective (x63) of the microscope.

Measurements were performed on an inverted fluorescent microscope and analyzed using ImageJ software. The components of a FRET microscope system included a light source to excite donor protein at its excitation wavelength, a beam splitter to divide the emission light of the donor and acceptor protein into two independent channels, and a camera to detect donor and acceptor channel intensities simultaneously (Figure 13). As described before ${ }^{239}$ a CoolLED single-wavelength light emitting diode was used as the light source for all FRET experiments. ARVMs with a good sensor expression were quickly selected using live fluorescent light mode manually to avoid photobleaching effects. The cyan fluorescent protein (CFP), integrated in our FRET biosensors as the donor protein, was excited at 440 $\mathrm{nm}$. The general emitted light from the cell was split into CFP and YFP emission channels using a DualView beam splitter and detected via a CCD camera in two separate channels. 


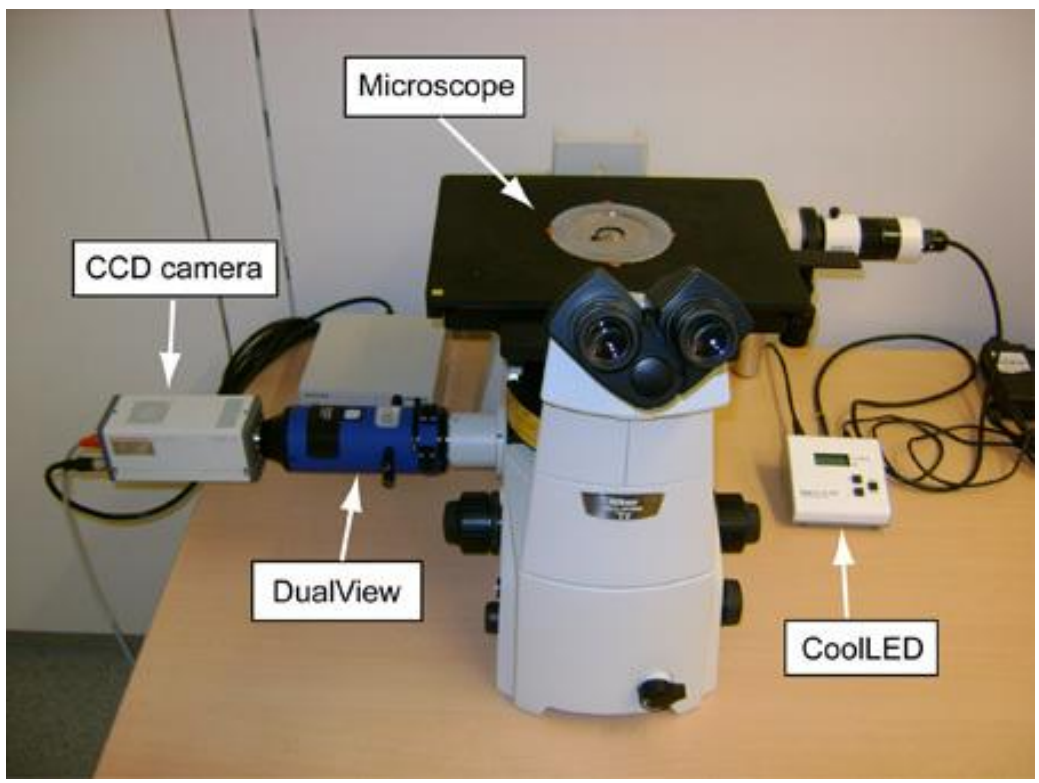

Figure 13. Layout of the FRET imaging setup comprised of a CoolLED, inverted Nikon microscope, DV2 DualView, and ORCA$03 G$ CCD camera. taken from Sprenger et al. ${ }^{239}$

Since viral expression of the biosensor protein inside the single cardiomyocyte vary from very strong to very low, sporadically showing perinuclear overexpression, cells with homologous fluorescence intensities are chosen for experiments. Perinuclear overexpression aggregates were excluded from the region of interest in all measurements. Upon adjusting the exposure time between $20-40 \mathrm{~ms}$ to have a relatively good signal-to-noise ratio, especially at the baseline, the images of the CFP and YFP emission channels were recorded every 5 seconds. As soon as a stable baseline had been reached, the compound dilutions at a volume of $400 \mu \mathrm{L}$ (or $300 \mu \mathrm{L}$ ) were gently added and changes of the YFP/CFP FRET ratio were monitored. The obtained raw data for CFP and YFP channel intensities were analyzed offline using Microsoft Excel and Origin 8.5 software. For precise quantification of the changes in intensities during a single measurement, the YFP/CFP intensity ratio was corrected for the bleedthrough ( 0.9 coefficient) of the donor fluorescence into the acceptor channel:

$\mathrm{FRET}_{\text {ratio }}=\left(\mathrm{YFP}_{\text {intensity }}-0.9 \times \mathrm{CFP}_{\text {intensity }}\right) / \mathrm{CFP}_{\text {intensity }}$

All data shown in section 3 are corrected FRET ratios calculated from the raw intensity data.

\section{2. 21 Statistics}

All data were analyzed using Origin Pro 8.5 software and shown in means \pm SE and differences between experimental groups were analyzed using the one-way ANOVA test. Differences were considered significant at $p<0.05$. 


\section{Results}

\section{1 Generation of the first version of the PLM-E1 FRET Biosensor}

In order to measure spatio-temporal regulation of cAMP dynamics in the vicinity of Phospholemman (PLM), we developed a FRET based biosensor derived from the cytosolic cAMP sensor E1-camps or simply E1-camps ${ }^{249}$ fused to the carboxyl terminus of the human PLM cDNA provided by Prof. Michael Shattock. The cytosolic E1-camps sensor is based on a single cAMP binding domain and was used as control in all FRET experiments. The reason to use Epac1-camps is its capability previously identified in other studies to show a larger FRET change upon cAMP binding in cells, although having a slightly lower affinity than some other cAMP sensor such as Epac2-camps ( 2 $\mu \mathrm{M} v s . \sim 1 \mu \mathrm{M})^{239}$.

The ${ }_{\text {human }}$ PLM sequence was fused to the YFP-Epac1-CFP sequence of E1-camps cDNA via Nhel restriction endonuclease site to create the plasmid PLM-E1 in the pcDNA3.0 vector. Additionally, the sequence between PLM and YFP contained a special linker consisting of 18 bp (encoding GSGSAS), which should ensure a proper folding of the sensor during the translation of the protein (Figure 14).

Hereupon, one had to test the functionality of the targeted cAMP sensor in living cells.

The pcDNA3.0 vector with its cytomegalovirus enhancer-promoter drives sensor expression in mammalian cells and simplifies its expression in HEK 293A cells after transfection with the PLM-E1 biosensor plasmid.

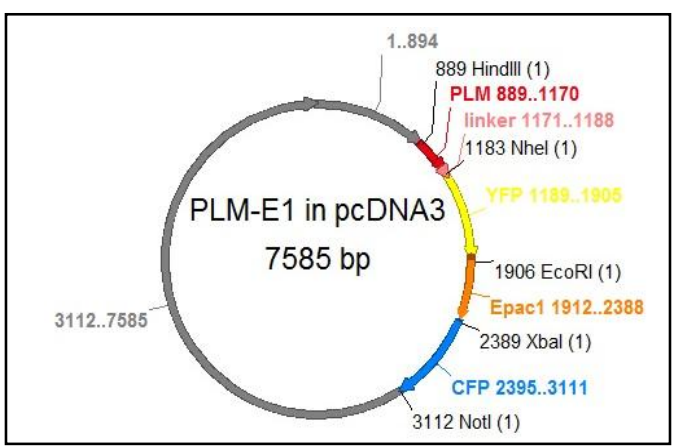

Figure 14. PLM-E1 construct in pcDNA3.0 vector.

The vector containing the PLM-E1 coding sequence contain a short linker (18 bp) GGA TCC GGA TCT GCT AGC between human PLM and YFP sequences. 


\section{1. 1 Expression and functional analysis}

First tests of transiently transfected HEK 293A cells were performed under a fluorescent microscope to analyze subcellular localization and to do first experiments of the FRET functionality of the targeted sensor. Epifluorescence images of PLM-E1 containing HEK293A cells showed a regional sensor expression pattern (Figure 15A), as expected. PLM-E1 localized at the plasma membrane and exhibited robust FRET. Upon $\beta$-adrenergic agonist isoproterenol (Iso) mediated cAMP stimulation, the PLM-E1 transfected HEK293A cells responded with a change in FRET ratio, proving its functionality in the sensor (Figure 15C).

SDS Page/Western blotting was carried out to check the size of the expressed protein. Based on the generated sequence, the PLM-E1 protein should have a theoretical size of approximately $75 \mathrm{kD}$. Immunoblot analysis of PLM-E1 transfected HEK293 cells under nonstimulated conditions were performed and investigated regarding relative expression level and molecular weight. Using anti-GFP antibodies, we observed bands corresponding to this theoretical weight for products expressed from the pcDNA construct including one additional band at about $20 \mathrm{kD}$ corresponding to the size of a monomeric GFP (CFP/YFP), indicating a possible partial degradation of the sensor protein (Figure 15B). Thus, our results indicate that the functional sensor construct is well expressed after transfection into HEK293 cells.

A

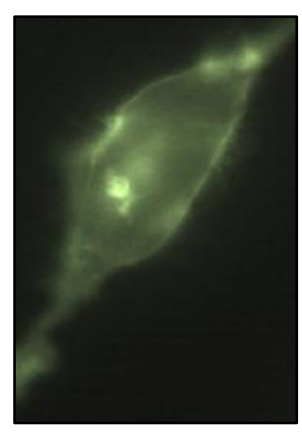

Figure 15. Expression, function and localization of PLM-E1 in HEK 293 cells.

(A) An epifluorescence image of a PLM-E1 transduced HEK 293A shows a predominant location on the plasma membrane, as expected.

(B) Immunoblot analysis of PLM-E1 protein ( 75

kD) expression after 24 hour transfection of increasing plasmid amounts into HEK293 cells probed with anti-GFP antibody. Blot is representative of at least three individual experiments. (C) FRET ratio trace recorded in HEK293A cells expressing PLM-E1. Isoprenaline (Iso) $100 \mathrm{nM}$ induced an increase in cAMP visualized by a decrease in YFP/CFP FRET ratio, proving good sensor protein functionality.

\section{B} PLM-E1 [ $\mu \mathrm{g}]$

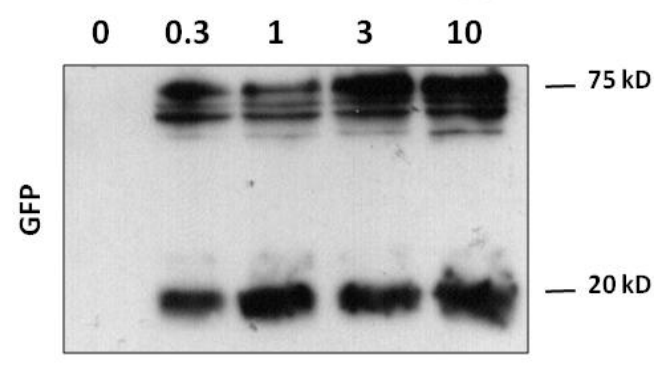

C

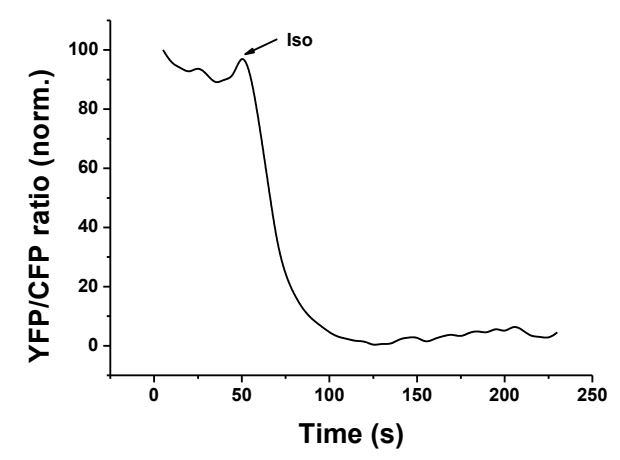




\section{1. 2 Localization analysis}

Since we plan to use the PLM-E1 as a tool to detect CAMP dynamics in the microdomain formed by PLM and the Sodium Potassium Pump in adult rat ventricular myocytes (ARVMs), it is obligatory to prove the physical association of the newly developed tagged PLM-E1 FRET biosensor with the $\alpha_{1}$-subunit $\left(\alpha_{1}-S U\right)$ of the NKA, comparable to endogenously expressed PLM in ARVMs. Using anti-NKA $\alpha_{1}$-SU antibodies immobilized on protein GSepharose beads, $\alpha_{1}$-SU was immunoprecipitated from PLM-E1 transfected HEK 293A cells, and all samples taken during the experiment were probed with anti-GFP antibodies. Interestingly, we could not detect any signal in the IP fraction (Figure 16A), likely due to the fact that although the biosensor is highly membrane localized, the expressed PLM-E1 protein is not associated with the a1-subunit. Troubleshooting for this problem led to optimization of the PLM-E1 construct with the already published caninePLM-YFP sequence, for which positive co-IP with the NKA has been published ${ }^{254}$. Of note, it is well known that HEK293 cells do not natively express any PLM. Considering this, it is not possible to detect any endogenous PLM in the following co-immunoprecipitation experiments.

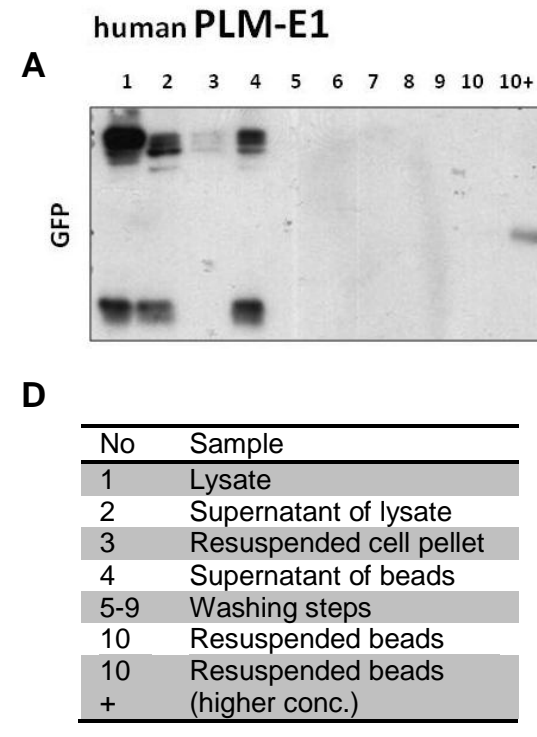

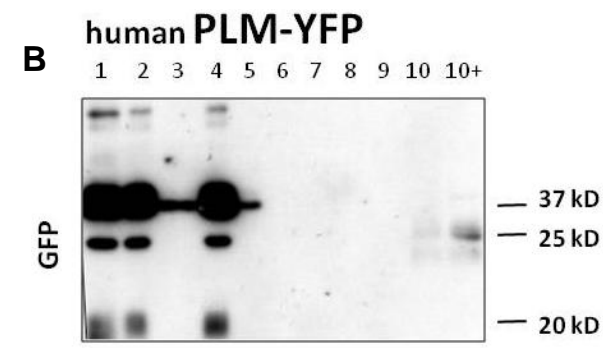

C canine PLM-YFP

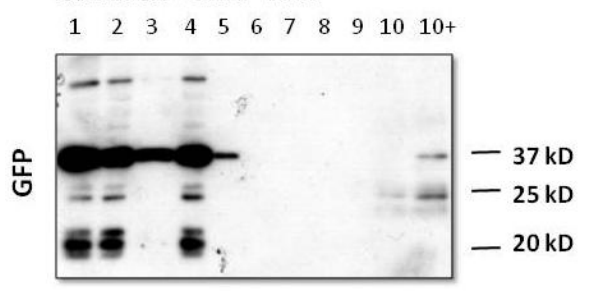

Figure 16. Co-immunoprecipitation by $1 \mu \mathrm{g} \alpha_{1}$-SU antibodies immobilized on Sepharose beads with the previously established protocol (see Methods) is shown. (A) PLM-E1 could not be immunoprecipitated from HEK 293A cells transfected with the PLM-E1 plasmid. (B) $24 \mathrm{~h}$ transfection of HEK 293A cells with human PLM-

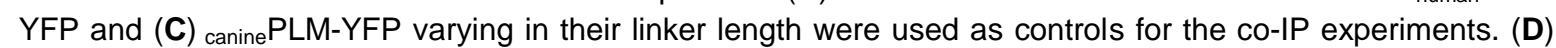
Immunoprecipitation starting material (No 1-3), material not immunoprecipitated (No 4: not IP-ed fraction) and immunoprecipitated material (No 5-10+: IP fraction) were separated on SDS-PAGE (10\% gel), gels were transferred to membranes immunoblotted as shown. The protein folding does influence the association of PLM-E1 with the sodium pump $\alpha_{1}$-subunit. caninePLM-YFP with a long linker shows a high capacity in binding to the $\alpha_{1}-S U$ of the NKA. 


\section{2 Generation of the optimized PLM-E1 FRET Biosensor and the adenoviral construct}

Despite of testing the functionality of the PLM-E1 and the clear change in CAMP induced FRET signal responses (Figure 15C) as well as the obvious membrane localization and translation of the developed PLM-E1 sensor protein (Figure 15A), it did not show any positive results regarding co-immunoprecipitation with the $\alpha_{1}-\mathrm{SU}$ of the NKA (Figure 16A). We considered a special caninePLM-YFP cDNA (kindly provided by Dr. William Fuller) as a possible alternative for FRET sensor backbone.

As mentioned in the last section, we also demonstrated that the canine PLM-YFP fusion protein construct is clearly associated with the $\alpha_{1}-S U$ of the sodium-potassium pump in HEK 293A cells (Figure 16C). It is inserted in a pEYFP N1 vector (Clontech, Paulo Alto, CA) and has a long linker sequence (60 bp) KRSRAQASNSAVDGTPVATG between the coding sequence for PLM and YFP.

Upon comparing both PLM-YFP sequences with different linker lengths (18 vs. $60 \mathrm{bp}$ ) in coIP experiments the canine PLM-YFP was found to built a physical interaction with the $\alpha_{1}$-SU of the NKA (Figure 16B,C). Therefore, this fusion protein was used in all further experiments to generate the adenovirus for transfection and FRET analysis of adult rat ventricular myocytes (ARVM).

To produce the recombinant PLM-E1 protein, the yellow fluorescent protein sequence was excised from the provided caninePLM-YFP containing pEYFP N1 vector via Kpnl (5'end) and Notl ( $3^{\prime}$ end) restriction endonuclease sites and ligated via triple-ligation at its C-Terminus with the complete YFP sequence amplified from the pEYFP N1 vector through PCR with designed primers flanking Kpnl and EcoRI sites and the Epac1-CFP sequence digested from the Epac1-camps cDNA by EcoRI and Notl to create the plasmid PLM-E1 in the given pEYFP N1 vector (Figure 17A).

As the objective of this project is to characterize $\beta$-adrenoreceptor ( $\beta$-AR) mediated cAMP dynamics in the PLM/NKA microdomain in ARVM, we created an adenovirus encoding E1PLM using the Invitrogen Gateway ${ }^{\circledR}$ Cloning System. The sensor sequence was amplified with specially designed primers containing flanking DNA recombination sequences called BP sites. After PCR amplification of an approximately 2255 bp product and gel purification the construct was cloned into an entry vector. The PLM-E1 construct was than subcloned into an expression vector using the LR Reaction based on DNA recombination. Here, the entry vector is combined with the destination vector called pAd_CMV_V5DEST (Figure 17B) and further used for amplification in HEK 293A cells. The expression vector contains human recombinant deficient (deletion in E3 gene) adenovirus type 5 with a cytomegalovirus promotor to drive protein expression of PLM-E1 in mammalian cells. 
A

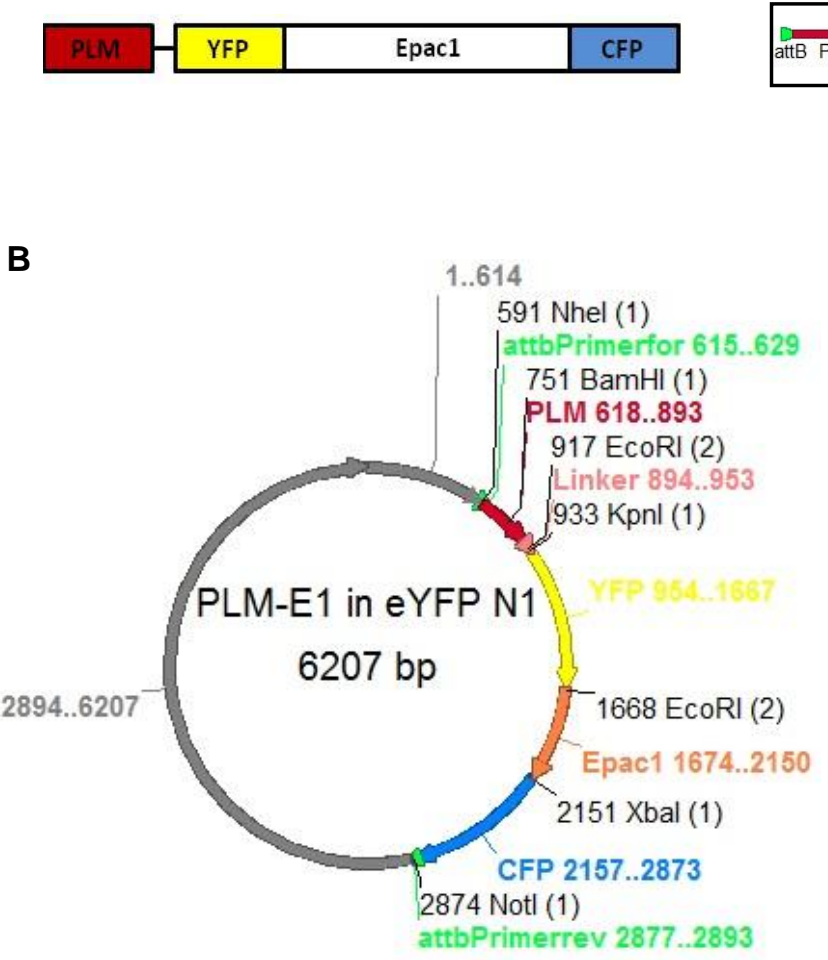

C

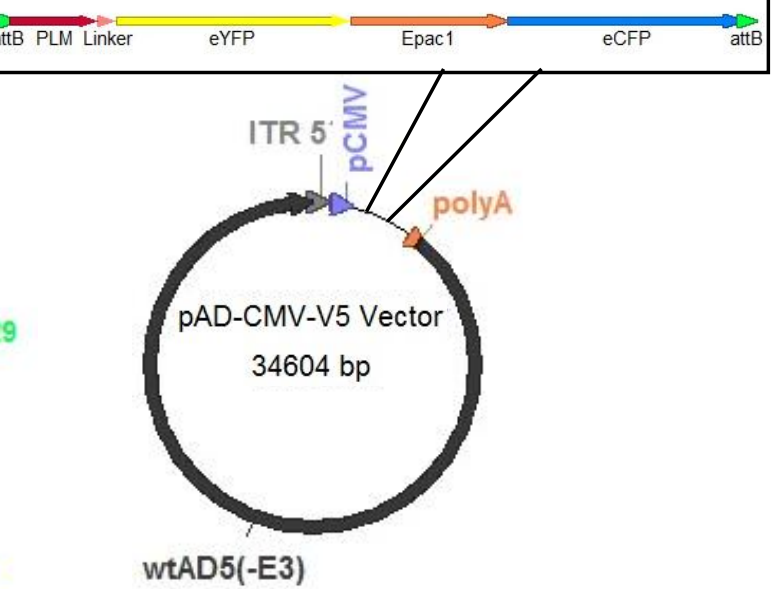

D

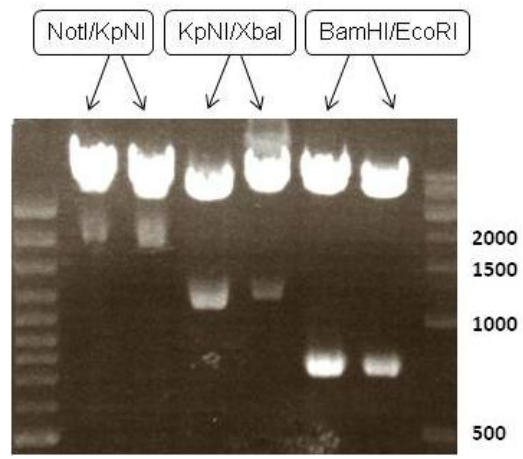

Figure 17. Design of the optimized PLM-E1 biosensor construct.

(A) Schematic representation of the PLM-E1 sensor construct that includes two fluorophores, yellow (YFP) and cyan fluorescent proteins (CFP), sandwiching the cAMP-binding domain Epac1 and fused to phospholemman (PLM). (B) pEYFP-N1 vector containing the coding sequence for PLM-E1 and showing attb forw. and rev. primer binding sequences used for further cloning steps into the final adenoviral vector. (C) Map of the recombinant adenoviral vector with the PLM-E1 sequence. It has a truncated version of human adenovirus type 5 and a CMV promoter for expression in ARVMs. (D) $1 \%$ agarose gel demonstrates DNA segments control digestion of the PLM-E1 construct in the pEYFP-N1 vector at 1900 bp (Notl/KpNI), at $1250 \mathrm{bp}(\mathrm{KpNl} / \mathrm{Xbal})$ and $750 \mathrm{bp}$ (BamHl/EcoRI).

\section{2. 1 Sensor characterization - expression}

To investigate molecular weight and the expression pattern of the developed PLM-E1 sensor, we transfected ARVMs with the PLM-E1 adenoviral construct at a range of $\mathrm{MOI}$ between 0 and 1000. The lysates were subjected to Western Blot analysis using anti-total PLM antibody and anti-GFP antibody. Based on the sequence of the sensor, its theoretical molecular weight is expected at $75 \mathrm{kD}$ (Figure 18B). Using the total PLM and the GFP antibody we detected a cluster of additional bands at $37 \mathrm{kD}$ and transfection rate dependent bands at $50 \mathrm{kD}$ and $22 \mathrm{kD}$, suggesting gene products originating from unexpected protein modification and trafficking in the Golgi apparatus. With regards to sensor expression, we examined confocal pictures of ARVMs transfected with the cytosolic E1-camps sensor, used for comparative real time measurements for cytosolic cAMP dynamics, versus the PLM 
tagged version PLM-E1, which should be spatially localized in the PLM/NKA microdomain. The genetically modified PLM-E1 sensor expresses the two fluorophores YFP and CFP like its predecessor E1-camps, which are recorded in separate detection channels with excitation wavelengths at $514 \mathrm{~nm}$ and $442 \mathrm{~nm}$ (Figure 18A).

A1

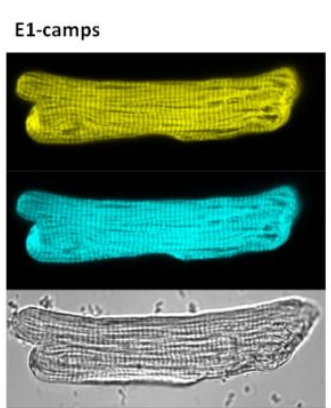

A2

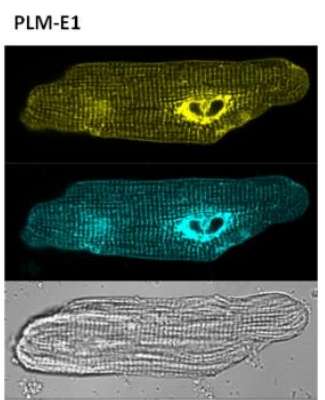

B

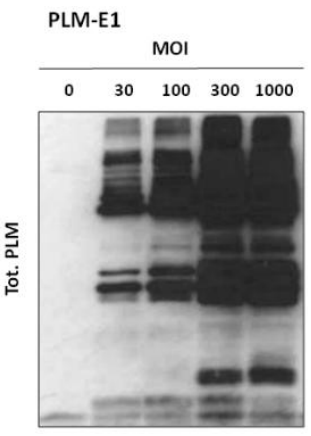

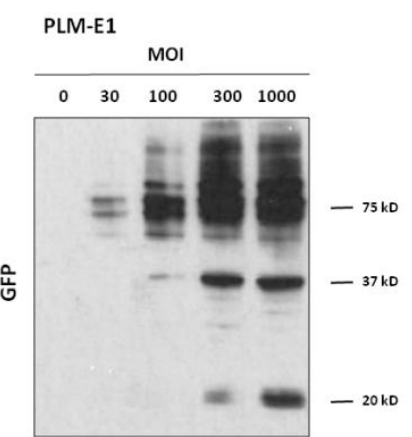

Figure 18. Expression of the PLM-E1 sensor. (A) Confocal images of AVRMs transfected with adenoviral constructs (A1) E1-camps and (A2) PLM-E1. YFP and CFP channels are recorded separately reveal a confined $Z$ line dominant pattern for the expression and localization of the targeted PLM-E1 compared to Epac1-camps, which is more evenly distributed in the cytosol and membrane parts. (B) Immunoblot expression analysis for PLM-E1 transfected ARVM. Cultured ARVMs were transfected for $48 \mathrm{~h}$ with the adenoviral vectors at $10,30,100,300$ or $1000 \mathrm{MOI}$. Cell lysates were subjected to SDS-PAGE $(10 \%$ gel) and Western blot analysis using an anti-total PLM (1:10000) and anti-GFP (1:160000) antibody. Blots are representative of at least three individual experiments.

In the confocal pictures, the PLM-E1 sensor revealed a predominant membrane localization, although there were always some aggregates observed in the perinuclear regions which could be excluded from analysis of live cell imaging data.

\section{2. 2 Sensor characterization - localization}

To insure accurate FRET measurements in the proper compartment of ARVMs, we carried out a biotin labelling and purifying procedure of cell surface proteins of PLM-E1 transfected ARVMs and saw a clear localization of the biosensor in the surface membrane fraction (Figure 19). The sensor was detected with an anti-GFP antibody against the fluorophores and appeared at $75 \mathrm{kD}$ in the surface membrane fraction alongside the $\alpha_{1}-\mathrm{SU}$.

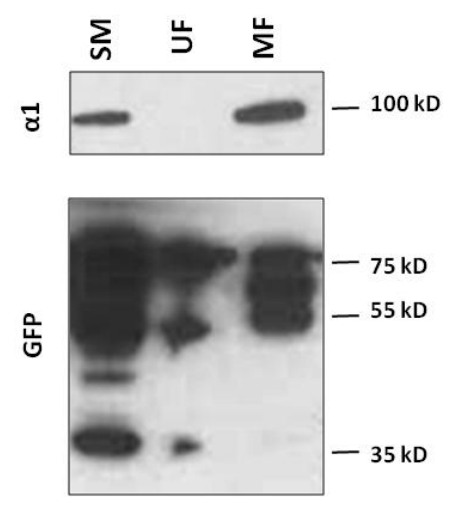

Figure 19. Cell surface biotinylation of PLM-E1 transduced

ARVM. PLM-E1 transfected ARVMs were briefly incubated with sulfo-NHS-SS-biotin and then incubated for $10 \mathrm{~min}$. Thereafter, cells were lysed in $1 \%$ triton $\mathrm{X}-100$ in PBS supplemented with protease inhibitors (lysis buffer) for $30 \mathrm{~min}$ at $4^{\circ} \mathrm{C}$. Biotinylated proteins that had not been degraded (Starting Material, SM) were purified ( $4 \mathrm{~h}$ to overnight at $4{ }^{\circ} \mathrm{C}$ ) using Streptavidin-Sepharose beads (GE Healthcare). Upon washes in lysis buffer and collecting the unbound fractions (UF), cell surface proteins in the membrane fraction (MF) were eluted in SDS-PAGE sample buffer supplemented with $5 \%(\mathrm{v} / \mathrm{v})$ -mercaptoethanol by heating at $60{ }^{\circ} \mathrm{C}$ for $10 \mathrm{~min}$. All samples were immunoblotted for PLM-E1 using anti-GFP antibody and sodium pump $\boldsymbol{\alpha}_{1}$-subunit. 
To prove the correct localization of the sensor in the PLM/NKA microdomain, confocal images of PLM-E1 transfected adult rat ventricular myocytes with a transfection efficiency about $80 \%$ (data not shown) were analyzed and revealed a T-tubular pattern and sporadically occuring perinuclear regions with enhanced fluorescence intensity (Figure 18A2). Compared to E1-camps, which is expressed throughout the entire cytosol with intense striations at the membrane (Figure 18A1), the PLM-E1 sensor is predominantly membrane bound as desired. To visualize the physical interaction of the $\alpha_{1}$-SU with its genetically modified regulatory sensor protein PLM-E1, we immunostained the $\alpha_{1}$-SU in PLME1 transfected cardiomyocytes. The colocalization analysis demonstrates that there is a nearly perfect overlay of the sensor signal (Figure 20B) as well as of the stained endogenous PLM (Figure 20A) signal with the $\alpha_{1}$-SU, indicating that the sensor localization corresponds to the endogenous PLM and NKA location.
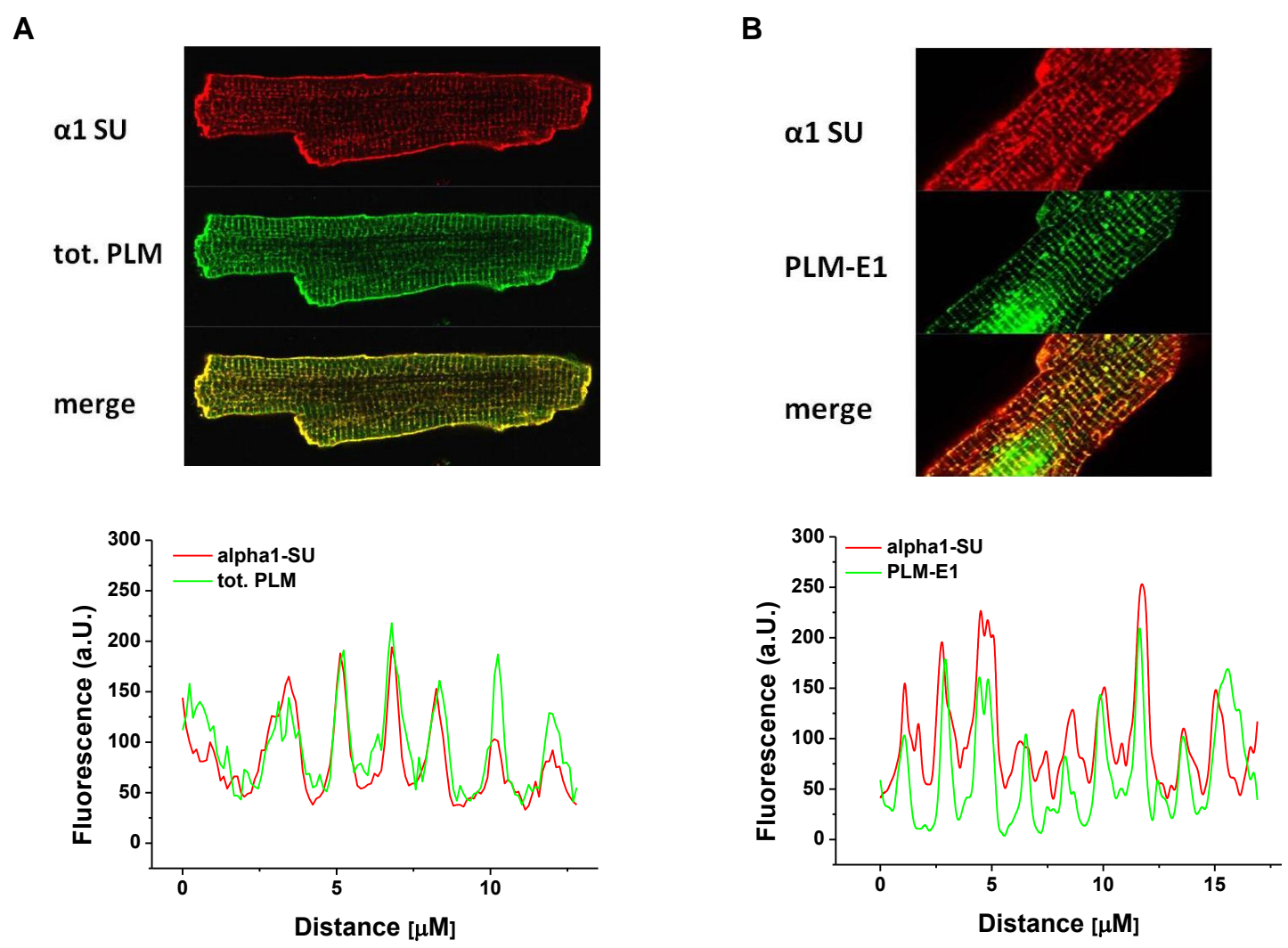

Figure 20. Co-localization of PLM-E1 with the sodium pump. Representative confocal images ( $\mathrm{n}>5$ for each) of immunostained (A) Wildtype and (C) PLM-E1 transfected (300 MOI) ARVMs. Co-localization of PLM with $\alpha_{1}-S U$ of the NKA is confirmed for endogenously expressed PLM and the overexpressed PLM-E1 by the intensity overlay of both fluorescent signals - see $\mathbf{A}$, bottom and $\mathbf{B}$, bottom.

With regards to biochemical evidence, co-immunoprecipitation experiments revealed the association of PLM-E1 with the $\alpha_{1}$-subunit in transduced ARVM and thus the correct localization of the sensor (Figure 21). 
For this purpose, we first conducted co-immunoprecipitation experiments with immobilised $\alpha_{1}$-SU antibodies on protein G-Sepharose beads to detect total phospholemman of PLM-E1 transfdunced ARVM. As expected, the beads catched endogenous phospholemman at $17 \mathrm{kD}$ and the expressed the biosensor at $75 \mathrm{kD}$ amongst other PLM including bands, characteristic for the sensor protein PLM-E1 (Figure 21A). Equally, we analysed E1-camps transfected ARVMs as a negative control, in which case only the endogenous PLM at $17 \mathrm{kD}$ could be pulled down from the lysed cells (Figure 21A).

To insure the association of PLM-E1 with the endogenous NKA- $\alpha 1-S U$, we have additionally used the GFP-Trap reagent (Chromotek), which has an excellent binding to fluorescent proteins, with no background or non-specific capture. Because the antibody is raised in lamas, one gets no interference of heavy and light lgG chains with sodium pump antibodies when immunoblotting. Compared to the E1-camps transduced ARVMs as a negative control, the $\alpha_{1}$-SU could be successfully pulled down almost entirely from the unbound fraction (No. 4) into the IP-fraction (No. 10; 10+) (Figure 21B). We looked further into the coimmunoprecipitated GFP fractions of the sensor protein and detected a strong GFP band in the IP fraction at the expected size of $75 \mathrm{kD}$. Additional bands at 37 and $22 \mathrm{kD}$ were detected, which are characteristic for PLM-E1 expression in cardiomyocytes, as indicated in the section 3. 2. 1.

A

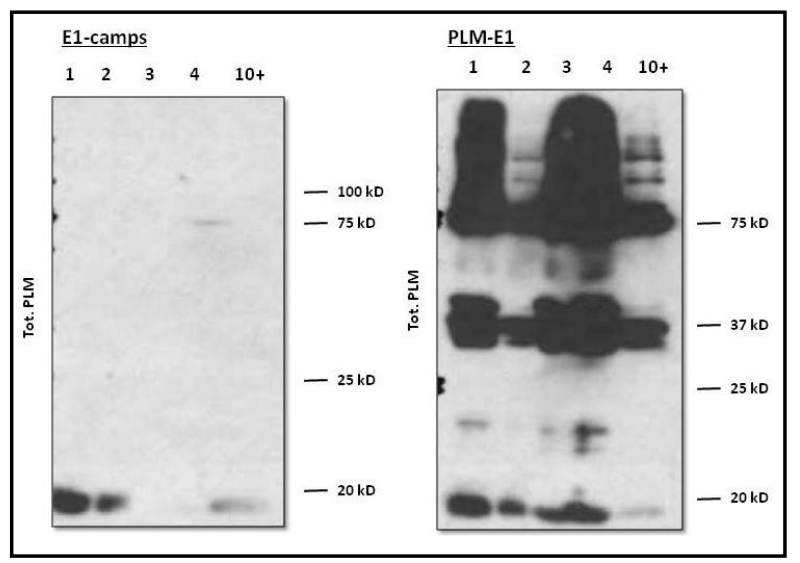

B

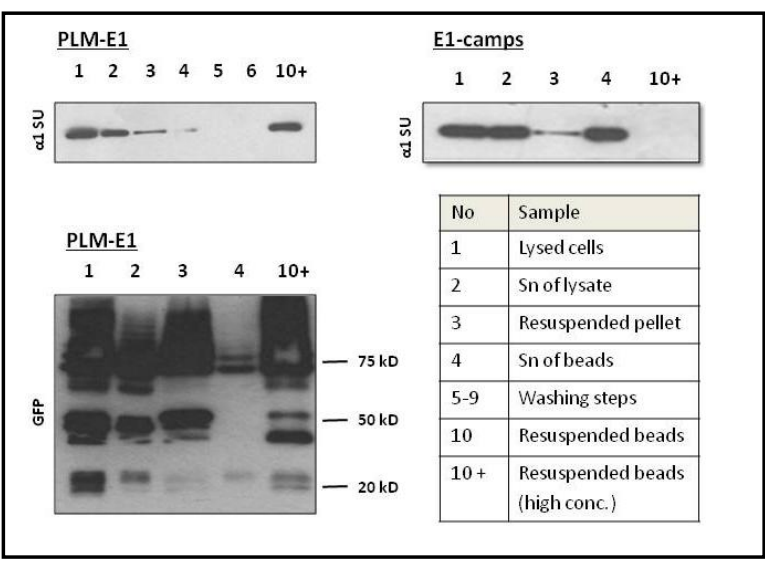

Figure 21. Association of PLM-E1 with the sodium-potassium pump a1 subunit.

(A) Representative immunoblots containing $\alpha_{1}-\mathrm{NKA}+\mathrm{PLM}$ colP analysis in PLM-E1 and E1-camps transfected ARVM. For co-IP of PLM-E1 and the $\alpha_{1}$-NKA, three wells ( 90.000 cells) of PLM-E1 and Epac1-camps (neg. control) transfected ARVM were used. Myocytes were lysed in $2 \mathrm{mg} / \mathrm{ml} \mathrm{C12E10} \mathrm{(Sigma)} \mathrm{in} \mathrm{PBS} \mathrm{supplemented}$ with protease inhibitor cocktail (Roche) and PhosStop (Roche) phosphatase inhibitor mixtures, insoluble material was removed by centrifugation at $17,500 \times g$ for $5 \mathrm{~min}$ at $4{ }^{\circ} \mathrm{C}$. Supernatants were agitated overnight at $4^{\circ} \mathrm{C}$ with anti-NKA- $\alpha_{1}$-subunit monoclonal C464.6 (Santa Cruz Biotechnology) preimmobilized on protein G-Sepharose beads (GE Healthcare). After multiple washes in $0.5 \mathrm{mg} / \mathrm{ml} \mathrm{C12E} 10$ in PBS, all samples taken during the immunoprecipitation procedure were eluted in SDS-PAGE sample buffer, separated by SDS-PAGE $(12 \%$ gel), transferred to membranes, and immunoblotted for total PLM.

(B) Representative Western Blots with immunoprecipitation experiments of fluorophores of PLM-E1 and Epac1camps transfected ARVM ( 90.000) precipitated using GFP-Trap (Chromotek) under identical conditions, and samples were immunoblotted as shown. All blots are representatives for at least three individual experiments. 


\section{2. 3 Sensor characterization - function}

For functional analysis of the inhibitory effect of the PLM-E1 sensor on NKA, we measured the ouabain-sensitive influx of radioactive ${ }^{86} \mathrm{Rb}^{+}$, thereby monitoring the NKA activity in HEK 293 cells stably expressing PLM-E1. Stable transfection of HEK293 with the sensor resulted in approximately $37 \%$ loss of NKA activity showing a siginificant dicrease in ${ }^{86} \mathrm{Rb}^{+}$uptake compared to a non-PLM-expressing HEK 293 line (Figure 22), indicating that the expression of PLM-E1 inhibits the sodium pump. Hence, sensor protein PLM-E1 is properly inhibiting the NKA, as the endogenous PLM does.

To test the FRET capability of the PLM-E1 sensor, we stimulated transduced ARVMs with the $\beta$-AR agonist isoproterenol (Iso) and monitored a clear decrease in YFP/CFP ratio as a consequence of the local microdomain CAMP increase, thus proving sensor functionality (Figure 23). Therefore, this new sensor could be ised in all futher FRET experiments.

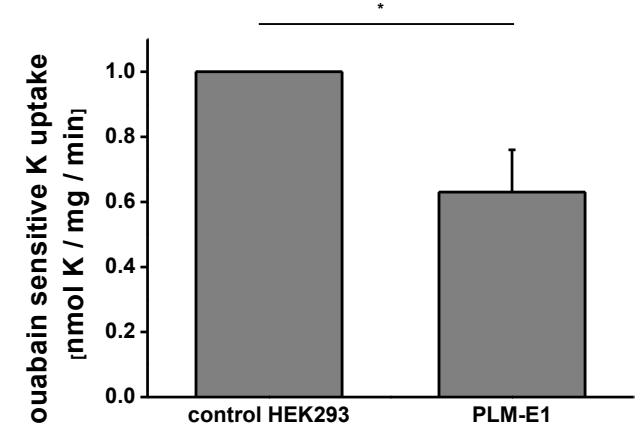

Figure 22. Inhibition of NKA activity by PLM-E1. Na pump activity was measured as ouabain-sensitive ${ }^{86} \mathrm{Rb}$ uptake by cells stably expressing PLM-E1. In this assay pump activity is expressed as a percentage of the activity in the same cell line which do not express PLM. PLM-E1 expressing HEK293 cells inhibit the sodium pump, HEK293 do not $\left(n=3\right.$; $\left.{ }^{*} P<0.05\right)$

A

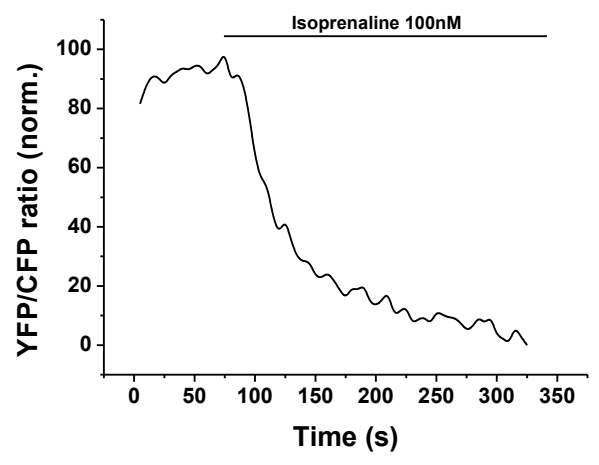

C

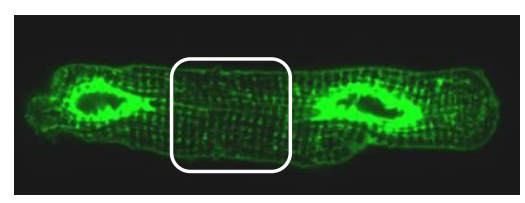

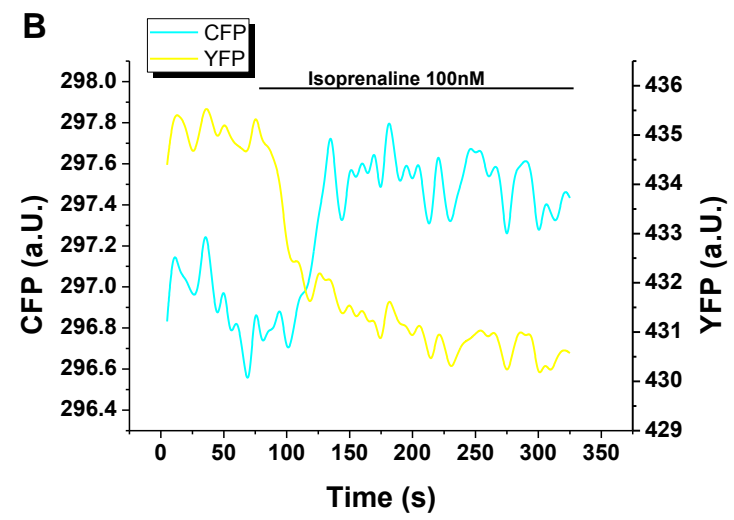

Figure 23. Sensor function in PLM-E1 transduced ARVMs. (A) Representative FRET signal trace recorded in a PLM-E1 transfected myocyte. Treatment with isoprenaline $100 \mathrm{nM}$ induces an increase in CAMP and a significant decrease in YFP/CFP ratio. (B) Recorded single YFP (acceptor) and CFP (donor) intensities for the same experiment. (C) Representative region of interest for a single FRET experiment in PLM-E1 transfected ARVM excluding strongly fluorescent perinuclear regions of the cell. 
The next step was to exclude PLM-E1 sensor affinity for CAMP and Isoprenaline sensitivity. Transduced cardiomyocytes were treated with the adenylyl cyclase inhibitor MDL12,330A to minimize basal intracellular cAMP before performing the concentration-response FRET experiments with the CAMP analogue 8-Br-2'-O-Me-CAMP-AM. In addition, the biosensor sensitivities were compared between the localized PLM-tagged and the cytosolic sensor in terms of FRET response to increasing concentrations of Iso.

We demonstrated that the membrane localized PLM-E1 sensor has a slightly lower affinity to CAMP than the cytosolic $\mathrm{E} 1$-camps $\left(\mathrm{EC}_{50}=4.1 \pm 1.1\right.$ vs. $0.9 \pm 0.2 \mu \mathrm{M}$, respectively) (Figure 24A). The concentration response dependency for Iso revealed a comparable sensitivity of PLM-E1 with the cytosolic E1-camps (Figure 24B). Hence, the non-tagged biosensor does not show a more intense perception of FRET changes in the cytosol than the localized sensor meaning that we could expect commensurate results for microdomain and cytosol in the following experiment.

A

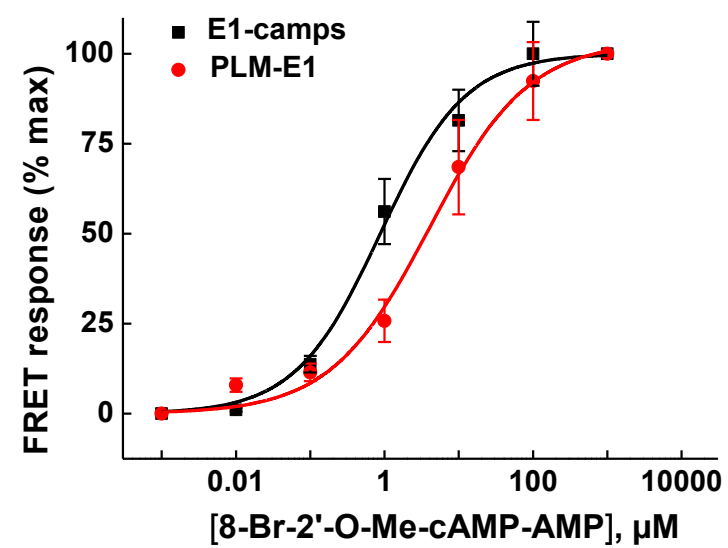

B

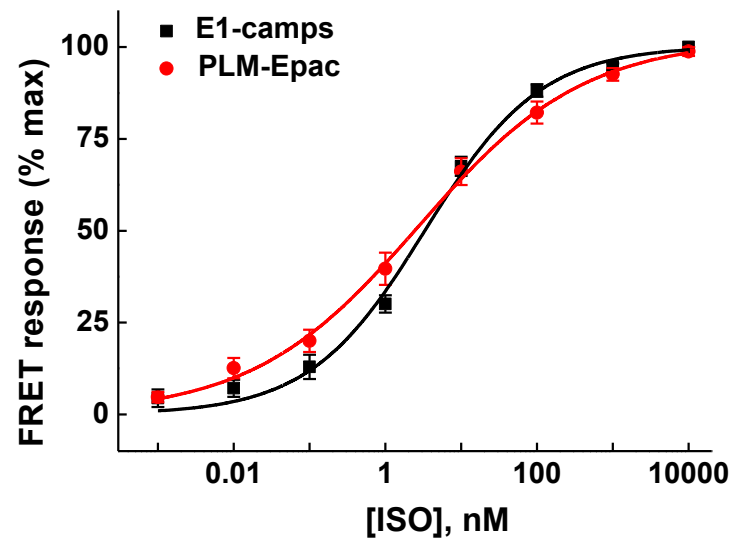

Figure 24. Sensitivity of E1-camps and PLM-E1 to cAMP and increasing isoprenaline (ISO) concentrations. (A) Concentration response dependencies measured in PLM-E1 and E1-camps transduced ARVMs pretreated with the adenylyl cyclase inhibitor MDL12,330A (100 $\mu \mathrm{M})$ for 10 minutes and treated with increasing concentrations of the cell-permeable cAMP analogue cAMP 8-Br-2'-O-MeCAMP-AM. PLM-E1 shows a 4-times lower affinity for than the cytosolic E1-camps biosensor (EC50= $4.1 \pm 1.1$ vs $E C 50=0.9 \pm 0.21 \mu \mathrm{M})$. Means $\pm S E, n=7-12$ cells from 4-5 rat hearts each. $(B)$ Isoprenaline induced FRET response for the Epac1-camps and the PLM-E1 sensor. Accordingly transfected ARVM treated with Iso concentrations ranging between $0.001 \mathrm{nM}$ and $1 \mu \mathrm{M}$ displayed no significant differences regarding the sensor Iso sensitivity for the targeted PLM-E1 and cytosolic E1-camps sensors $\mathrm{EC}_{50}$ values were $4.4 \pm 1.2$ and $4.3 \pm 0.7 \mathrm{nM}$, respectively. Means $\pm n=15$ and 10 cells from 4 rat hearts each. 


\section{3 Comparative FRET analysis of E1-camps and PLM-E1 transduced ARVMs}

Phospholemman exerts its inhibitory effect on the $\mathrm{Na}^{+} / \mathrm{K}^{+}$-ATPase only when it is not phosphorylated by kinases, for example at its Ser-68 residue by the PKA, which is downstream of the adrenergic $\beta_{1}$ - or $\beta_{2}$-adrenergic receptor subtypes.

\section{3. 1 Effects of individual $\beta$-AR subtypes}

To investigate which subtype is involved in the receptor-cAMP signaling pathway in the PLM/NKA microdomain, we performed the following FRET-based experiments. Individual $\beta$ $A R$ subtypes were selectively stimulated in E1-camps and PLM-E1 transduced ARVMs of 810 weeks old rats. To do so, we inhibited the $\beta_{1}$-AR with CGP-20712A (100 nM) and the $\beta_{2^{-}}$ AR with $\mathrm{ICI} 118551(50 \mathrm{nM})$ and stimulated the cAMP production with Isoprenaline (100 $\mathrm{nM})$. As a result, in the cytosol, we could detect proportionally the same signal strength in $\beta_{1}-A R$ FRET response as in the PLM microdomain. In contrast, we measured a significantly higher amount $(\sim 65 \%)$ of $\beta_{2}$-AR-CAMP induced FRET ratio change in the PLM/NKA microdomain compared to the cytosol (Figure 25E). $\beta_{2}$-AR signals have been previously demonstrated to be localized at T-tubular membranes and to be highly locally confined ${ }^{29}$. This data correlates with the displayed expression pattern of PLM-E1 sensor (Figure 18A2) and its ability to particularly well distinguish between the previously shown global $\beta_{1}$-AR and localized $\beta_{2}-A R$ cAMP pools ${ }^{28}$.

A

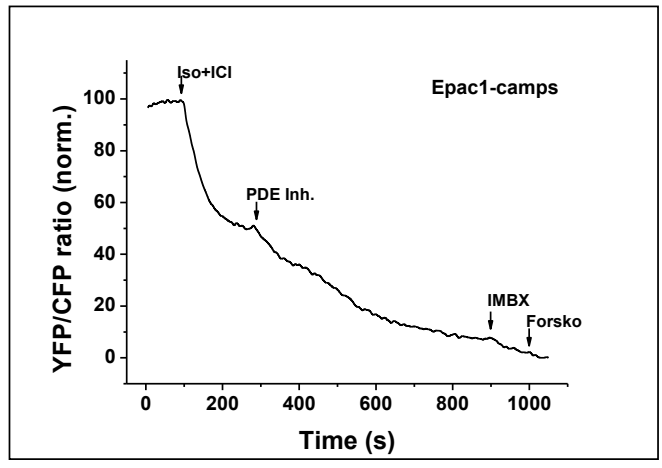

C

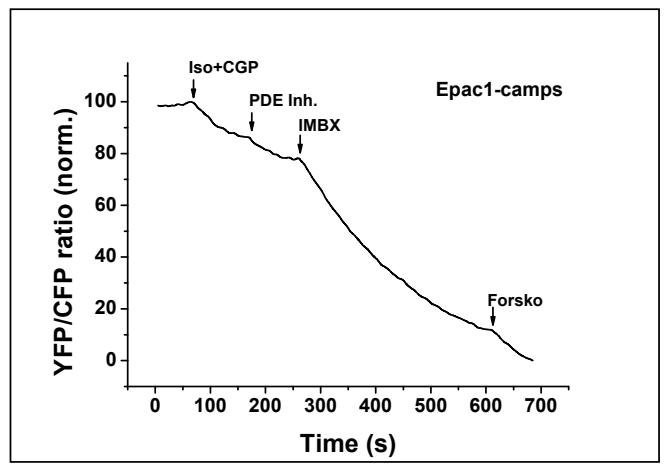

B

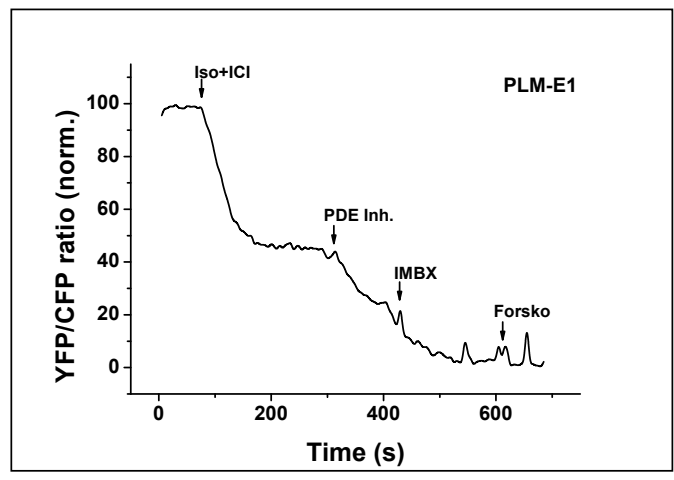

D

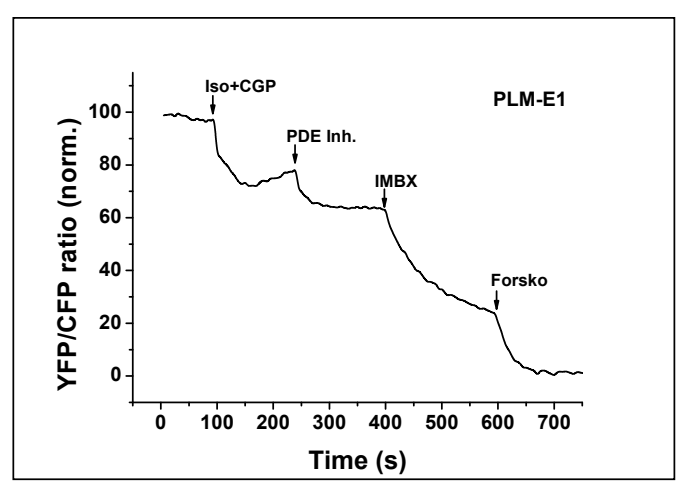


E

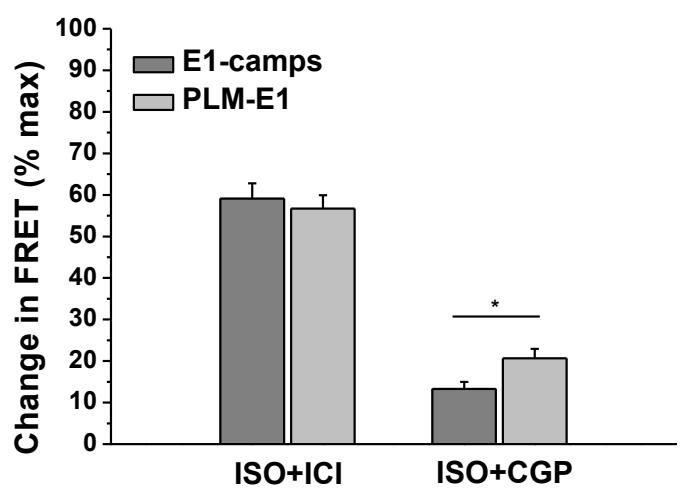

Figure 25. Predominant control of $\beta_{2}$-AR over the PLM/NKA microdomain.

$(\mathbf{A}, \mathbf{B})$ Representative FRET traces for E1-camps and PLM-E1 expressing rat cardiomyocytes upon $\beta_{1}$-AR-selective stimulation (100 nM ISO + $50 \mathrm{nM}$ of the $\beta 2$-AR blocker ICI 118551). (C,D) Representative FRET traces from Epac1-camps and PLM-E1 rat cardiomyocytes after $\beta_{2}$-ARselective stimulation (100 nM ISO $+100 \mathrm{nM}$ of the $\beta 1$-AR blocker CGP-20712A). $\beta_{1}$-AR stimulation showed no significant difference in FRET response between bulk cytosol and the PLM/NKA compartment. Maximal stimulation of adenylyl cyclase was achieved by selective PDE inhibitors used in different concentrations described in the next section, the unselective PDE inhibitor IBMX (100 $\mu \mathrm{M})$ and the direct adenylyl cyclase activator forskolin $(10 \mu \mathrm{M})$. (E) Quantification of the FRET experiments. Selective $\beta_{2}$-AR stimulations strongly rise cAMP the PLM/NKA microdomain and resulted a significant difference to the cytosolic FRET response. $\beta 1$-AR stimulation did not show a different percentage in max. FRET responses in the PLM/NKA microdomain compared to bulk cytosol. Means $\pm S E, n=30-40$ cells from 3-4 rat hearts per condition.

\section{3. 2 PDE Profiles in PLM-E1 and E1-camps transduced ARVMs}

It is well known, that through local activities of specific phosphodiesterases (PDEs), cAMP in the heart is organized in distinct microdomains. To investigate the organization of cAMP signal transduction in the PLM/NKA microdomain, we performed a series of experiments to analyze the regulation of cAMP dynamics via PDE families in the vicinity of Phospholemman versus the cytosol. Since the low availability of the inhibitors for PDE1, 8 and 9 prohibited further investigation in terms of their role in modulation of cyclic nucleotide signaling in the PLM/NKA microdomain and as the predominant CAMP degrading PDE subtypes in the heart are PDE2, 3 and 4, we focused on the latter ones. Looking at basal actions of the Phosphodiesterases and their control of local cAMP microdomains, we measured FRET responses without $\beta-A R$ stimulation of ARVM transduced with PLM-E1 and E1-camps. We used specific inhibitors for PDE2, 3 and 4 without prestimulating with Iso and continued with a general PDE blocker IBMX and further increase the cAMP production, adding Iso and Forskolin (Forsko) as the last steps until we fully saturated the FRET sensor. For calculations, we related the data to the maximal stimulation signal of the sensor. Interestingly, we detected no significant difference between any PDE family in E1-camps cells, but an important contribution of PDE2 in the PLM microdomain (Figure 26C), which 
seems to be strongly regulating basal cAMP levels in close proximity of PLM, as compared to the general cytosolic level of FRET response. In rat cardiac myocytes, splice variants of the PDE2 isoform, especially PDE2A2 and PDE2A3 have been found as preferentially associated with functional membrane structures, such as plasma membrane ${ }^{114}$, which could be responsible for the regulation of local cAMP pools in the vicinity of PLM.

A

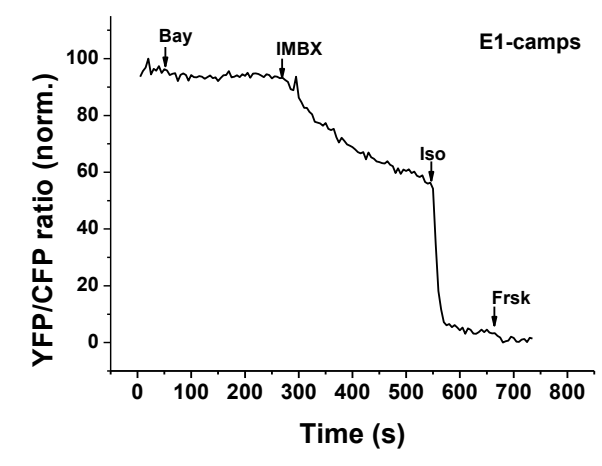

C

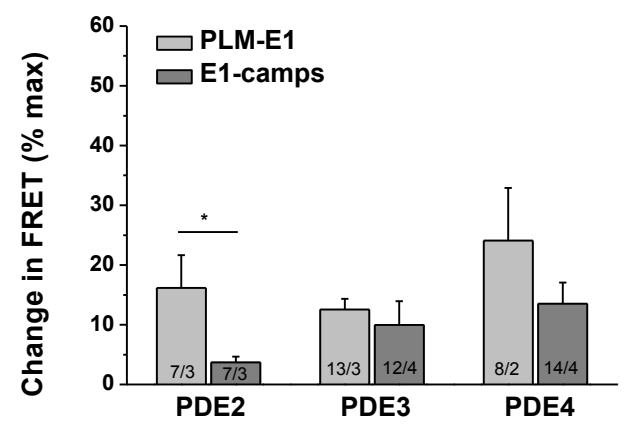

B

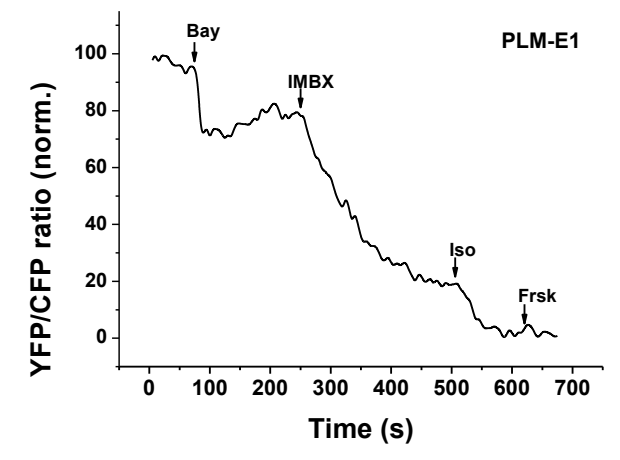

Figure 26. Significant PDE2 contribution to basal cAMP degradation in the vicinity of PLM. Representative FRET traces upon basal PDE2 Inhibition of Epac1-camps (A) and PLM-E1 (B) transduced 16 weeks old ARVM. Upon specific PDE inhibition either with BAY 60-7550 for PDE2 (100nM), Cilo for PDE3 $(10 \mu \mathrm{M})$ or Roli for PDE4 $(10 \mu \mathrm{M})$, CAMP accumulation in the cell is achieved through inhibition of all PDEs via IBMX $(100 \mu \mathrm{M})$ and continued with maximum stimulation of the cell by Iso $(100 \mathrm{nM})$ and Forsk $(10 \mu \mathrm{M})$ in order to saturate the sensor. (C) Quantification of FRET experiments as related to the maximum stimulation uncovered PDE2 as the PDE family crucial for confining the PLM/NKA compartment from the bulk cytosol. Means $\pm S E, n$ cells from $\mathrm{N}$ rat hearts per condition is written on the bars. ${ }^{*}$ - significant differences at $p<0.05$.

In the next set of experiments, we focused on the effects of PDE Inhibition in the presence of isoproterenol. Under selective stimulation of $\beta_{1}$ - and $\beta_{2}$-ARs, we analyzed the PDE profiles of PDE2, 3 and 4 after subtype specific $\beta$ - adrenergic stimulation in the microdomain versus the cytosol and could not find any differences amongst the groups regarding the $\beta_{1}$-subtype stimulation (Figure 27C). Looking at the FRET responses to individual PDE inhibitors after $\beta_{2}$-AR stimulation, we discovered that cAMP dynamics in the vicinity of the membrane protein Phospholemman are predominantly regulated via PDE3 in the PLM/NKA microdomain (Figure 27D). The localized PLM-E1 sensor detected an up to two-fold higher FRET signal change in the microdomain, in comparison to cells which were treated with PDE2 or PDE4 
inhibitors after selective $\beta_{2}$-AR stimulation. The obtained results were precisely calculated as the ratio of the PDE Inhibitor effect over the general PDE Inhibition by IBMX.

A

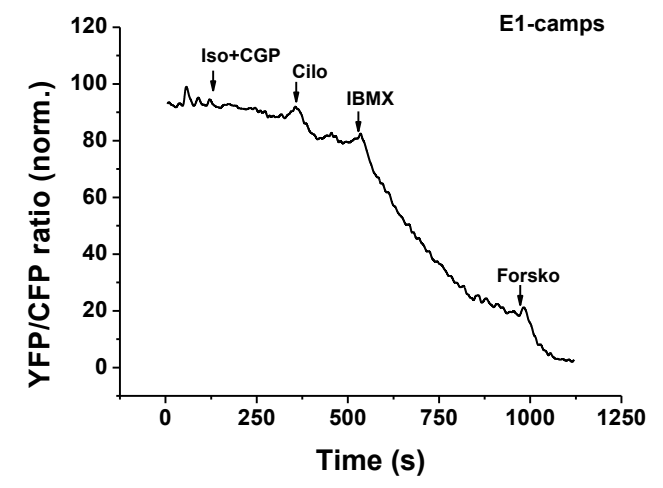

C

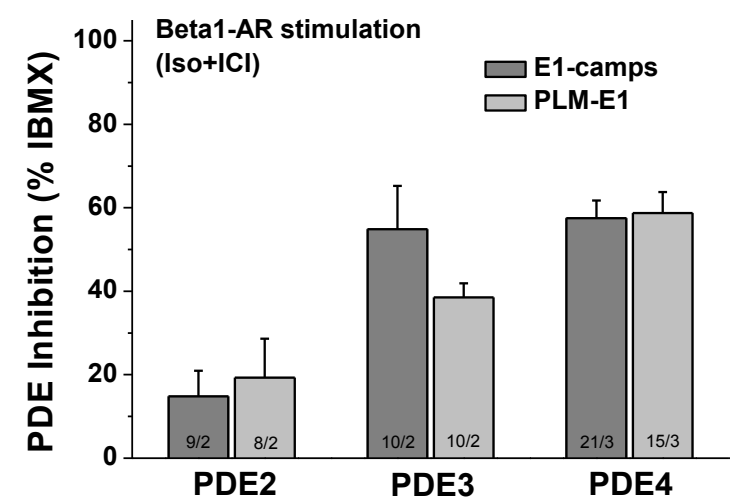

B

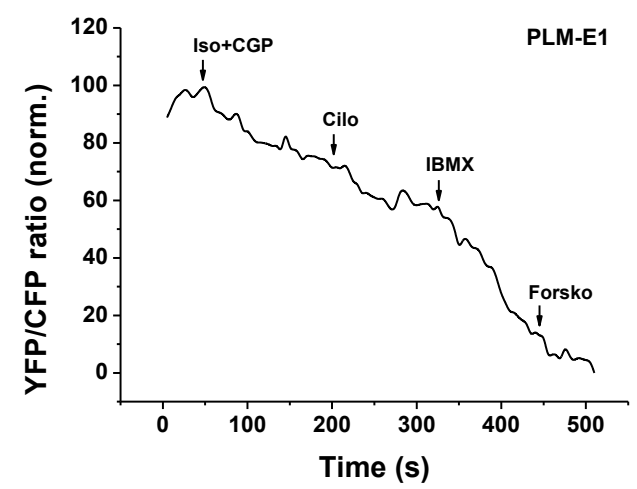

D

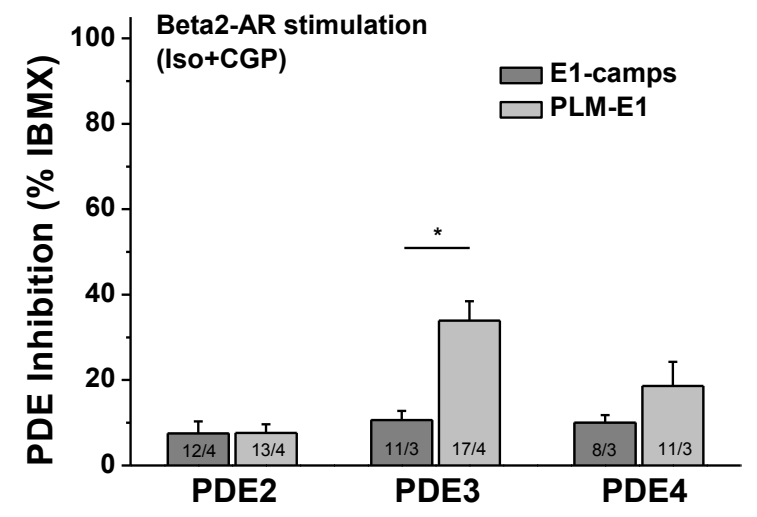

Figure 27. PDE3 pool in the vicinity of PLM confines $\beta_{2}$-AR mediated cAMP signals to the PLM/NKA microdomain. Representative FRET ratio traces demonstrating PDE3 effects in E1-camps (A) and PLM-E1 (B) transduced ARVMs after $\beta_{2}$-AR stimulation with Iso+CGP. (C, D) Quantifications of the PDE Inhibitor signals as a \% of the general PDE inhibitor IBMX effect. There is a markedly increased response to PDE3 Inhibition after selective $\beta_{2}$-AR stimulation in the PLM/NKA compartment in contrast to the bulk cytosol. ${ }^{*}$ - significant differences at $p<0.05$. Shown are means $\pm S E, n=$ cells from $\mathrm{N}=$ rat hearts per condition written on the bars. Substance concentrations: ISO $100 \mathrm{nM}$, IBMX 100 $\mu \mathrm{M}, \mathrm{BAY}$ 60-7550 $100 \mathrm{nM}$ (PDE2 inhibitor), cilostamide $10 \mu \mathrm{M}$ (PDE3 inhibitor), rolipram $10 \mu \mathrm{M}$ (PDE4 inhibitor).

\section{3. 3 ANP/cGMP mediated PDE3 response in the PLM/NKA microdomain}

Since there is an indication, that there is a $\beta_{2}$-AR mediated cAMP pool controlled by local PDE3 activity in the PLM/NKA microdomain, we tested whether the stimulation of the cGMPproducing ANP receptors in PLM-E1 transduced ARVMs have any effect on CAMP levels. cGMP can activate PDE2 through by an allosteric mechanism. It can also inhibit PDE3, since cGMP acts as a competitive inhibitor of the cAMP hydrolysis by this PDE (for CAMP/cGMP crosstalk see Introduction, section 1. 1. 3). Indeed, quantification of all experiments (Figure 29C) as well as the representative traces for $\beta_{2}$ AR (Iso+CGP) prestimulated PLM-E1 cells 
(Figure 29A,B), demonstrate that $\beta_{2}$-AR-triggered FRET signals could be further decreased by the addition of ANP and abolished via Cilo-pretreatment (Figure 29B). PLM and PDE3 colocalization was analyzed by confocal imaging experiments with cells from 8-10 weeks old animals. Thus, freshly isolated ARVM were immunostained with PDE3 and total PLM specific antibodies (Figure 28A) to study the degree of the overlay (Figure 28B). Good colocalization of PDE3 with the endogenous Phospholemman could be confirmed, which supports the FRET results in this section.

A

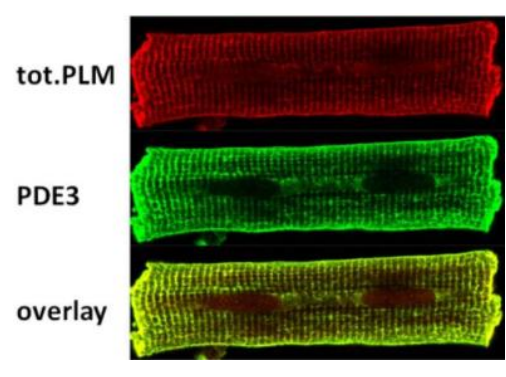

B

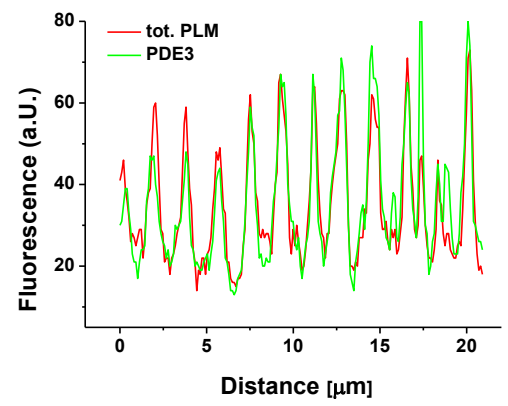

Figure 28. Co-localization of the PDE3A isoform with the PLM microdomain. (A) Representative ARVM immunostained with specific antiPLM and anti-PDE3 antibodies (B) showing a high degree of colocalization $(n>10)$.

A

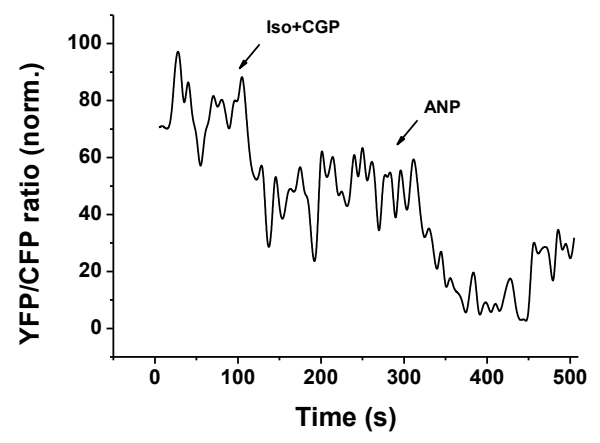

C

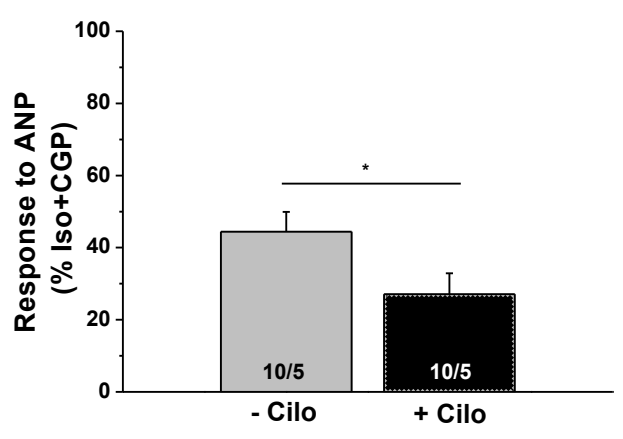

B

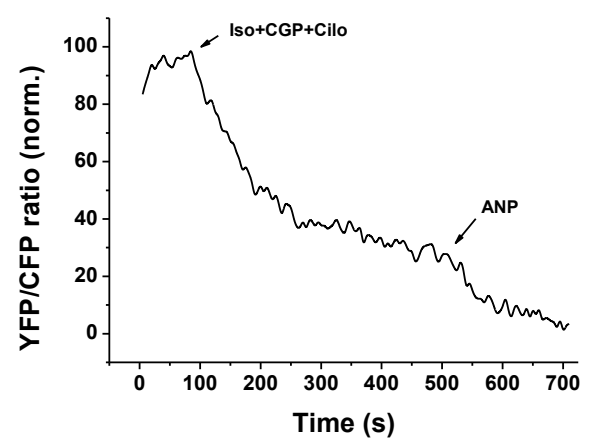

Figure 29. ANP induced PDE3 inhibition in ARVM results in high levels of $\beta_{2}$-AR/cAMP in the PLM/NKA microdomain. (A) Treatment of PLM-E1 transfected ARVM with atrial natriuretic peptide (ANP, $100 \mathrm{nM}$ ) after selective $\beta_{2}$-AR stimulation (Iso+CGP) leads to further accumulation of CAMP in the vicinity of PLM, (B) which is abrogated by Cilo $(10 \mu \mathrm{M})$ pretreatment, indicating a PDE3-dependent effect.

(C) Quantification of FRET ratio change to ANP treatment of PLM-E1 ARVM shown in \% Iso + CGP with and without pretreatment with Cilo. * - significant differences at $p<0.05$. Shown are means $\pm S E, n=$ cells from $\mathrm{N}=$ rat hearts per condition written on the bars. 


\section{3. 4 FRET measurements in PLM-E1 and E1-camps transfected ARVMs}

Considering the important pathological changes in $\beta$-AR - cAMP - PKA signaling at the subcellular level, we investigated possible alterations of spatiotemporal cAMP regulation in the vicinity of Phospholemman in ARVMs isolated from animals after myocardial infarction (MI). Sprague-Dawley rats were used to generate the progressive chronic heart failure model by ligation of the left anterior descending coronary artery via a suture. 16 weeks after $\mathrm{Ml}$ surgery, heart function was significantly decreased in MI vs. aged matched control (AMC) group as measured by echocardiography (Table 6).

Table 6. Phenotyping of aged matched control and 16 weeks post MI hearts. The results were calculated from M-mode echocardiography recordings (Vevo 770 micro-imaging system, Visualsonics) as a marker of alterations in wall contractile functionality. Additionally, the heart weight to tibia length (HW/TL) ratio was measured after isolation of the respective hearts as an indicator of progressive hypertrophy.

\begin{tabular}{|l|l|l|l|l|l|l|}
\hline \multirow{2}{*}{ Parameter } & \multicolumn{2}{l|}{$\begin{array}{l}\text { 24 weeks old aged matched } \\
\text { control (AMC) }\end{array}$} & $\begin{array}{l}16 \text { weeks post myocardial } \\
\text { infarction (MI) }\end{array}$ \\
\cline { 2 - 8 } & Value & $\mathrm{p}$ & $\mathrm{N}$ & Value & $\mathrm{p}$ & $\mathrm{N}$ \\
\hline Ejection fraction [\%] & $84.3 \pm 1.5$ & -- & 3 & $57.0 \pm 5.6$ & $\mathrm{p}<0.05$ & 4 \\
\hline $\begin{array}{l}\text { Heart hypertrophy } \\
{[\mathrm{mg} / \mathrm{mm}]}\end{array}$ & $29.5 \pm 0.5$ & -- & 3 & $36.8 \pm 2.3$ & $\mathrm{p}<0.05$ & 4 \\
\hline
\end{tabular}

\section{3. 5 Alterations in local PDE dependent cAMP regulation in the PLM/NKA microdomain}

We found out that the communication between the $\beta_{2^{-}}$AR and the PLM/NKA microdomain is predominantly driven by PDE3 in wildtype (WT) 8-12 weeks old Wistar rat hearts (Figure 27D). For that reason, we tested if this communication is deteriorated during the pathological state of myocardial infarction. We isolated cardiac cells from 16 weeks post-MI Sprague Dawley rats as control animals and transduced them with the PLM-E1 and E1-camps biosensor adenoviruses. After preblocking of the $\beta_{1}$-AR with CGP-20712A, we stimulated PLM-E1 transfected MI myocytes with Iso and looked at the different PDE inhibitor contributions by relating them to the proportion of IBMX induced FRET signal decrease. We demonstrated, that upon $\beta_{2}$-AR stimulation, PDE3 hydrolyzing activity is downregulated in the PLM/NKA microdomain. Concomitantly, PDE2 activity at the sarcolemmal PLM/NKA domain is upregulated (Figure $30 \mathrm{~F}$ ). There was no significant change in PDE contribution in response to $\beta_{2}$ mediated cAMP detected using the cytosolic E1-camps sensor (Figure 30E). 
A

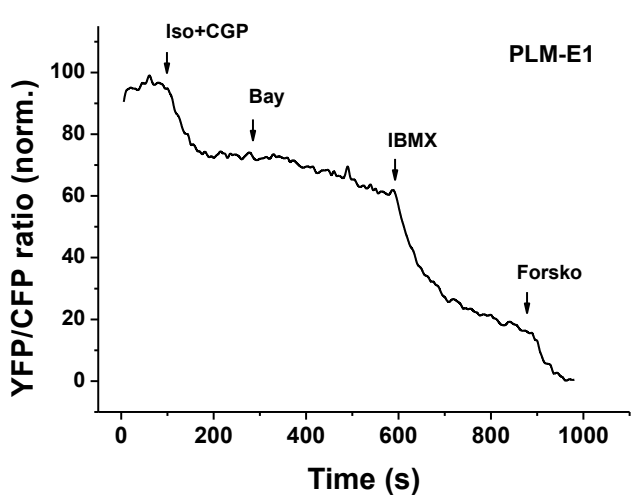

C

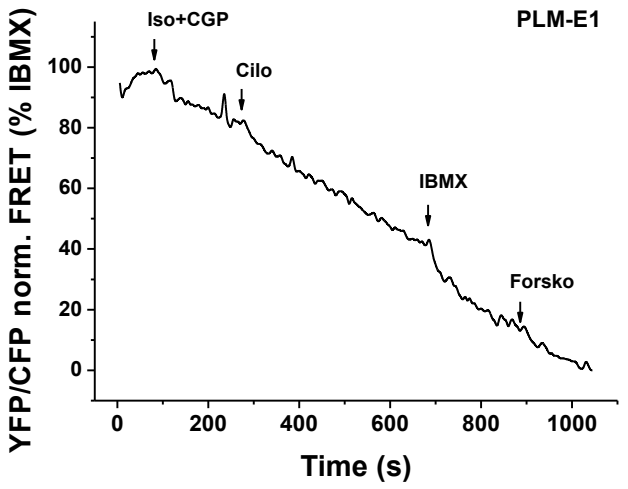

E

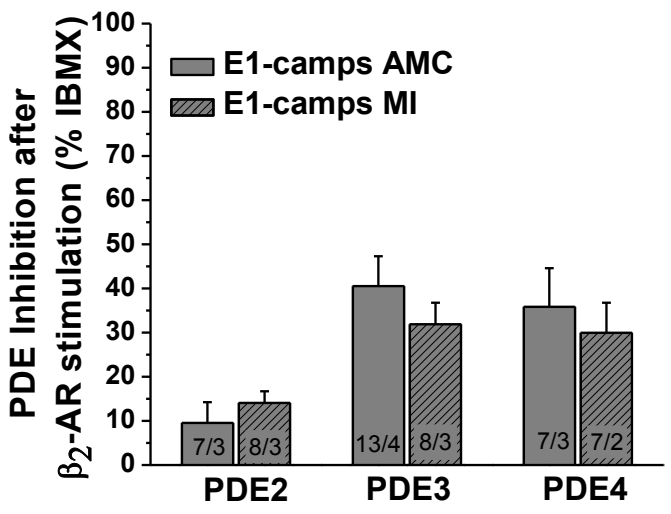

B

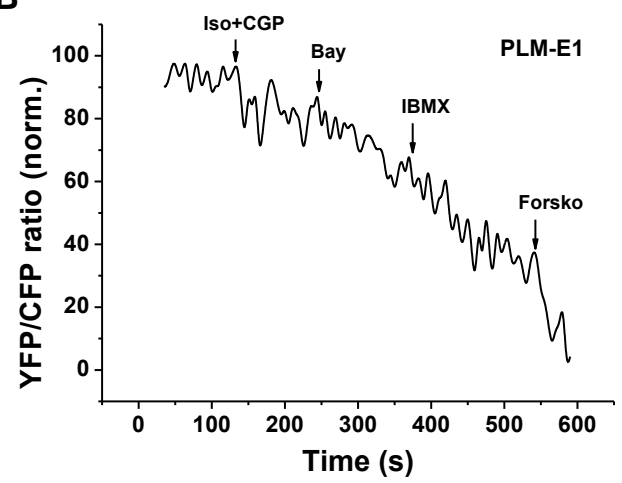

D

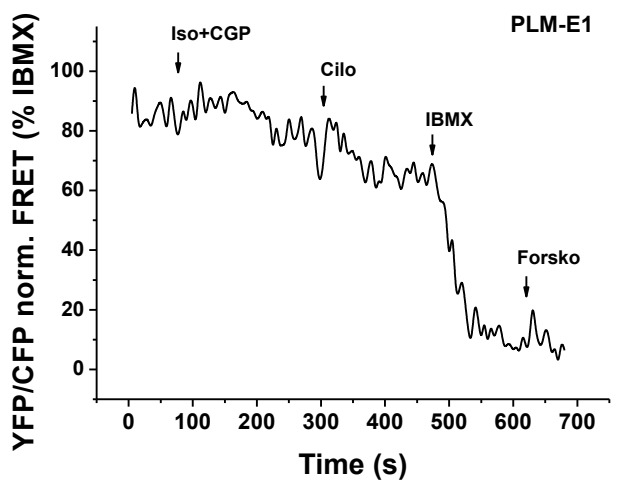

$\mathbf{F}$

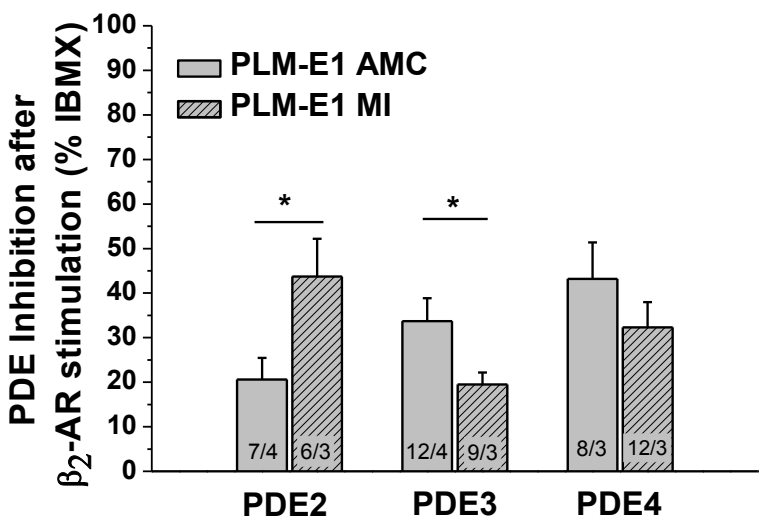

Figure 30. Contributions of individual PDEs to CAMP hydrolysis after $\beta_{2}$-AR stimulation in AMC and MI cardiomyocytes. (A,C) Representative FRET traces from AMC and (B,D) Ml cardiomyocytes expressing PLM-E1, treated with $100 \mathrm{nM}$ Iso+CGP for $\beta_{2}$-AR stimulation and subsequently with PDE2 inhibitor BAY 607550 (Bay, $100 \mathrm{nM}$ ) or PDE3 inhibitor cilosamide (Cilo, $10 \mu \mathrm{M}$ ), respectively. (E) Quantification of cytosolic (E1-camps) FRET experiments revealed no significant changes of any PDE contribution after MI. (F) Quantification of PLM-E1 specific (PLM-E1) FRET experiments showed a significant increase of PDE2 contribution in the microdomain of Ml cells, while PDE3 response was strongly decreased. Means $\pm \mathrm{SE}$, $\mathrm{n}=$ cells from $\mathrm{N}=$ rat hearts per condition. ${ }^{*}$ - significant differences at $\mathrm{p}<0.05$. 


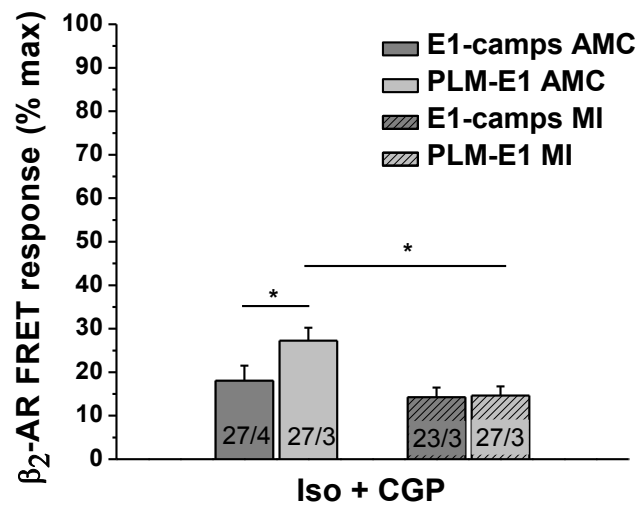

Figure 31. $\beta_{2}$-AR effects in AMC vs. MI cells. Comparison of the magnitudes of Iso (100 nM) responses measured with the targeted PLM-E1 and cytosolic E1-camps in an experiment performed as described in Figure 14. These data suggests a serious loss of receptor signal to the microdomain after $\mathrm{Ml}$ regarding $\beta_{2}$ adrenoreceptor signals. Means $\pm S E$, $\mathrm{n}=$ cells from $\mathrm{N}=$ rat hearts per condition. * - significant differences at $p<0.05$.

We checked for possible alterations in $\beta_{2}$-AR signal responses (Figure 31 ). Interestingly, the MI cells did no longer show a difference between cytosolic and PLM-E1 microdomain characteristic responses to Iso. The experiments revealed alterations regarding $\beta_{2}$-subtype specific cAMP responses, suggesting that the $\beta_{2}$-receptor-microdomain communication is seriously impaired in myocardial infarcted hearts.

\section{3. 6 cAMP degrading PDE3 pools in the PLM/NKA microdomain are delocalized in the hypertrophied heart}

Since we showed that $\beta_{2}-A R / C A M P$ signals are confined in the PLM/NKA microdomain through PDE3 activity, we focused on defining the alterations of this confined signaling in the chronic heart failure disease model. PDE3 dependent subtype specific $\beta_{2}-A R$ responses were dramatically diminished in hypertrophied heart cells compared to FRET responses in 24 weeks old aged matched control (AMC) rat heart cells (Figure 30F). We demonstrated the loss of PDE3 localization in the microdomain by immunostaining cardiomyocytes isolated from hearts after myocardial infarction with total PLM and PDE3A subfamily specific antibodies (Figure 32). Quantification of confocal images using the Pearson's coefficient, which is an indicator for the degree of co-localization, uncovered a disturbed localization of PDE3 in the microdomain in hypetrophied cells compared to aged matched control myocytes (0.85 vs. 0.92 ) (Figure 32 ). It was no more locally confined and in comparison to healthy aged matched control cells visibly altered. 


\section{tot.PLM PDE3 overlay}

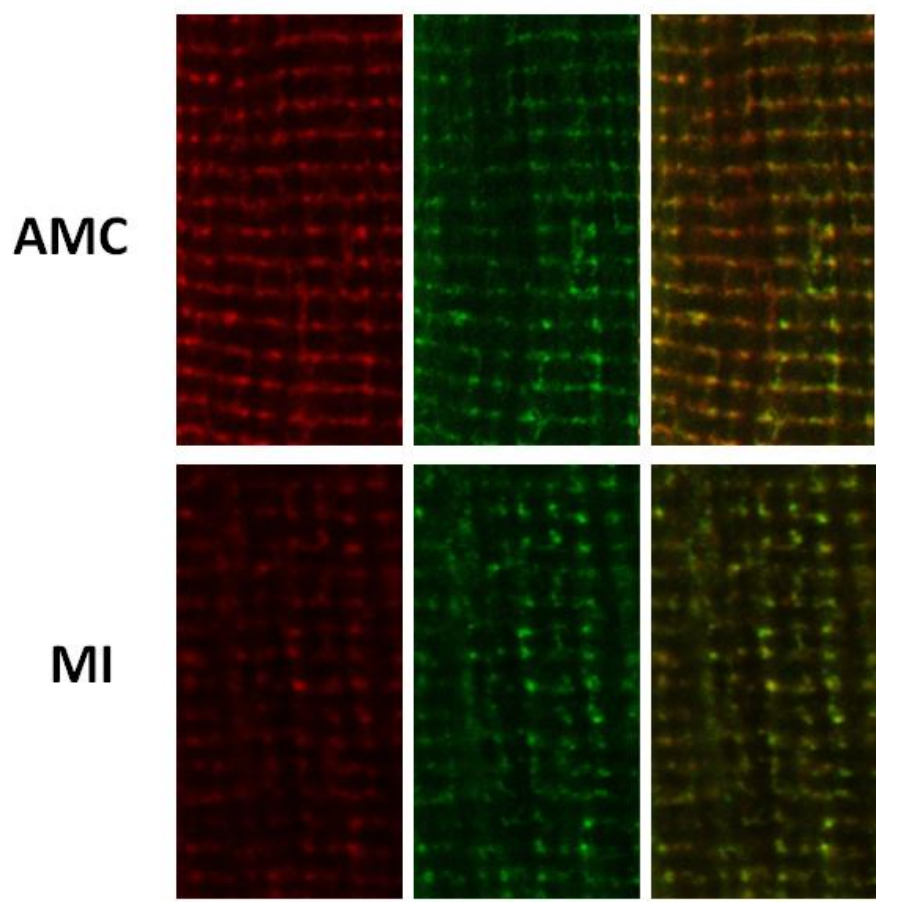

Figure 32. Confocal microscopy analysis of PDE3 localization. Aged matched control and $\mathrm{Ml}$ cells were immunostained with the PDE3 family specific antibody. Significant delocalization of locally confined PDE3 in hypertrophied cardiomyocytes were detected. Means $\pm \mathrm{SE}, \mathrm{n}=$ cells from $\mathrm{N}=$ rat hearts per condition. * - significant differences at $p<0.05$.

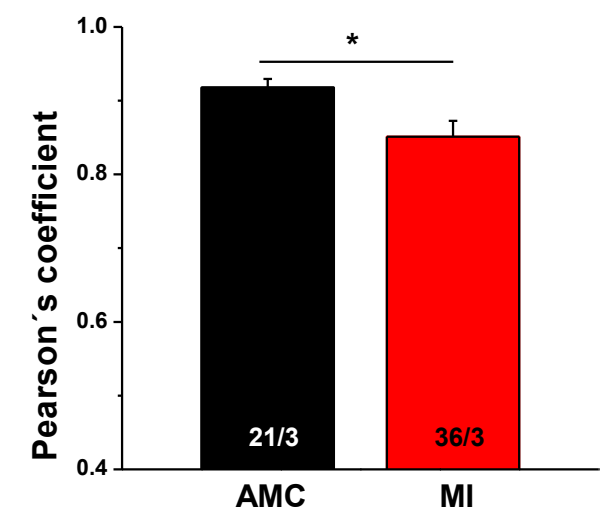

However, when the same protocol for the ANP FRET experiments (3. 3. 3) were applied during our analysis for MI-ARVM, we could not detect any clear FRET signals, so that it was not possible to conclude if there are better or worse ANP responses compared to aged matched control cardiomyocytes (data not shown). 


\section{Discussion}

\section{1 Challenges in generating novel PLM-E1 FRET biosensors with correct structural and functional properties}

The orchestrated action of specific subsets of PDEs, AKAPs, PKA subtypes and other scaffolding proteins involved in creating a local cAMP signalosome in subcellular microdomains has been proposed by numerous studies from different labs (see section 1. 1 . 3). The well-accepted model of cAMP compartmentation can explain the observed different physiological events operated by the same second messenger. Alterations in NKA activity and PLM expression ${ }^{230,255}$ are known to be associated with cardiac disease and are probably connected with a modification of CAMP/PKA dependent NKA regulation. In order to directly monitor cAMP dynamics at the PLM/NKA compartment and their changes in heart failure, the PLM-E1 FRET-based biosensor was generated. PLM-E1 is a targeted version of the cytosolic Epac1-camps ${ }^{249}$ (E1-camps) biosensor which carries the full-length PLM sequence on its $\mathrm{N}$-terminus. Initially, it was attempted to generate the recombinant DNA encoding for the PLM-E1 biosensor by tagging the CAMP sensitive single chain biosensor E1-camps to the human PLM via a 18 bp long linker region located between PLM and the YFP moiety (Figure 14).

Before generating the recombinant adenovirus with the PLM-E1 insert, non-viral HEK293 cell transfection served as a more 'easy-to-handle' gene delivery method to test functionality and localization of the sensor. Consistent with previous observations for the predominantly membrane expressed YFP-PLM ${ }^{205,254}$, the inclusion of the PLM sequence in the E1-camps construct showed an expected distribution of the targeted sensor exclusively at the plasma membrane (Figure 15A). Furthermore, the PLM-E1 sensor showed a correct expression pattern (Figure 15B) upon titration using Western blot with anti-GFP antibodies; the bands observed corresponded to the respective molecular weight ( $74 \mathrm{kD})$. The additional protein structure at $20 \mathrm{kD}$ might have been cleaved off during intracellular trafficking. However, we demonstrated that this had no effect on the functionality of the sensor (Figure 15C). The PLM-E1 transfected HEK293 cells showed a clear decrease in the FRET response triggered by Iso mediated intracellular cAMP elevation. Although the optical and functional results were as expected, the localized sensor did not co-immunoprecipitate with the endogenously expressed NKA- $\alpha$-SU (Figure 16A) from HEK293 cells in contrast to positive controls (Figure $16 \mathrm{~B}, \mathrm{C})$. This behavior of the sensor construct did not comply with the requirement for the PLM/NKA microdomain specific cAMP measurements. This discrepancy could potentially be caused by inaccurate folding of the protein of this size as we used a relatively short linker to connect the sequences of PLM and YFP. The optimized version with a 60 bp linker based on canine PLM-YFP (Figure 17B), which was successfully used in FRET studies before ${ }^{205}$, 
showed equal expression properties like humanPLM-E1 in HEK293 cells (data not shown). According to the cDNA sequence, dog and rat PLM are highly homologous ${ }^{256}$, so there was no concern about the compatibility of canine PLM-E1 FRET sensor in ARVMs. While working with titer controlled adenoviral gene delivery systems promises $100 \%$ transduction efficiency, such in vitro studies require a high quality cardiomyocyte population and successful cell isolation and culture ${ }^{257}$. Using an adenoviral vector to transfer the genetically modified biosensor sequence into cardiomyocytes is an efficient method which does not require damaging treatments for cells such as electroporation, somewhat similar to transgenic animal models ${ }^{154,251,258}$. This adenovirus mediated gene transfer approach has been used in many studies to demonstrate discrete spatial and temporal compartmentalization of CAMP in adult cardiac myocytes ${ }^{65,111,155,259-261}$. A clear advantage of in vitro adenoviral system studies is that there is no possibility for adverse effects on cardiac morphology and function, as compared to transgenic animals. Additionally, in our experiments the adenoviral transduction had no effect on cell viability or morphology of the cultured cells compared to non transfected cells. Upon generating the adenoviral vector with PLM-E1 as insert (Figure 17C), it was possible to characterize the sensor in ARVMs. It is confirmed in several studies that cardiac PLM resides at the surface membrane and its phophosphorylation status plays an important role in the regulatory effect on the $N_{K A}{ }^{166,262}$. Similarly, confocal imaging experiments of PLM-E1 transduced ARVMs (Figure 18A2 and Figure 20B) revealed thin parallel striations in correspondence of the sarcomeric $Z$ lines where endogenous PLM (end. PLM) is also expressed next to the NKA- $\alpha_{1}$-SU (Figure 20A). Despite of the observations of strongly fluorescent perinuclear clustering of the sensor in ARVMs, it could be demonstrated that there is enough protein expressed at the cell surface which co-immunoprecipitates with the NKA- $\alpha_{1}$-SU (Figure 21). Detailed sensor expression analysis with the tot. PLM antibody revealed bands corresponding to the respective molecular weights of the PLM-E1 sensor and end. PLM, indicating that not all of end. PLM was replaced by the sensor (Figure 18B). The assessed inhibitory effect of the sensor PLM on the NKA was in agreement with the end.

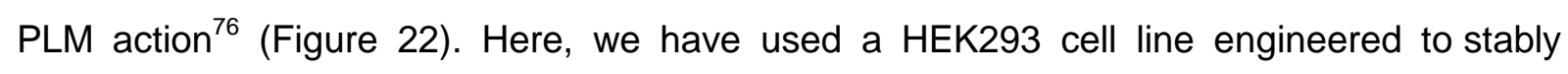
express PLM-E1, as experiments with transiently transfected cells may be confounded by transfection efficiency.

After adenoviral gene transfer of the PLM-E1 FRET biosensor into ARVMs, excitation of CFP at $436 \mathrm{~nm}$ and YFP at $514 \mathrm{~nm}$ led to robust fluorescent signals in both channels, proving the successful integration of donor and acceptor fluorescent proteins into the adenoviral FRET construct (Figure 18A2). Excluding areas of sporadically occurring strongly fluorescent perinuclear regions, presumably emerging from adenoviral overexpression and incorrect intracellular trafficking of the sensor, PLM-E1 was capable of generating a strong FRET signal in response to an elevation in intracellular cAMP concentration (Figure 10). This was 
similar to the unimolecular cytosolic E1-camps sensor which has been known to have a relatively large FRET ratio change and a high signal-to-noise ratio ${ }^{248}$. The kinetic properties of PLM-E1 are comparable to those of the E1-camps sensor (Figure 24), allowing FRET measurements with a high dynamic range at saturation (i.e. $100 \mathrm{nM}$ ) and subsaturating concentrations of Iso, which was particularly suitable for comparative measurements of PDE effects in the vicinity of PLM and bulk cytosol.

\section{2 Comparative FRET analysis of healthy ARVMs expressing adenoviral PLM-E1 and} E1-camps biosensors reveal differentially regulated local $\beta$-adrenergic signaling

Molecular mechanisms involving cAMP/PKA actions in the vicinity of PLM are known to be highly important in regulating cardiac function ${ }^{170}$. Especially during $\beta$-adrenergic stimulation PLM is an indispensable regulator of sodium and indirect calcium efflux ${ }^{190}$, whose absence and changes in regulatory properties may lead to alterations in cardiac function ${ }^{230,255}$. In this part of the study, the successful implementation of the localized FRET-based cAMP sensor PLM-E1 for direct monitoring of cAMP dynamics exclusively in sarcolemmal PLM/NKA microdomain could be demonstrated. Using this approach, it became possible to characterize the highly compartmentalized signals in the vicinity of PLM.

\section{2. 1 Restricted cAMP diffusion to the NKA/PLM complex via basal activity of phosphodiesterases}

It was observed that in PLM-E1 transduced ARVMs without concomitant cAMP stimulation, inhibition of PDE2 significantly raises CAMP as detected by the cAMP sensitive locally expressed biosensor (Figure 26C). Although the overall PDE2 action in cardiac myocytes is small compared to PDE3 and PDE4 activities $^{65}$, its contribution in cAMP degradation in the vicinity of PLM might be of great importance. This almost three-fold higher local PDE2 responses compared to FRET changes monitored by the cytosolic E1-camps sensor is the first direct evidence of PDE2 confining the PLM microdomain from the bulk cytosol. The basal PDE2 action in the PLM microdomain may serve to protect PLM in the subcellular compartment from excessive phosphorylation. All three PDE2 splice variants have been characterized to be located either at the cytosolic or at membrane fractions of cardiac myocytes. At the plasma membrane, there is a predominant control of PDE2A2 and PDE2A3 isoenzyme activities where it can hydrolyze both cAMP and CGMP and regulate the second messengers in a compartment-specific manner ${ }^{91}$. Indeed, it was shown that PDE2 is involved in cGMP dependent CAMP regulation of LTCCs of frog ventricular myocytes and human atrial cardiac myocytes ${ }^{91,263,264}$. PDE2 controlled cAMP might play a role in PLM phosphorylation and therefore in NKA regulation. 
It is noteworthy that PDE2 enzyme activity is stimulated by cGMP (as mentioned in chapter 1. 1. 3). Whether cGMP-mediated, PDE2-dependent decrease of basal cAMP blunts PLM phosphorylation and NKA activity remains to be defined. There is still a debate if cGMP is involved in PLM phosphorylation ${ }^{219,265}$. Madhani et al. have reported that the PLM-Ser69 phosphorylation could contribute to sildenafil (PDE5 inhibitor) induced cardioprotection against reperfusion injury via a NO/cGMP dependent pathway. Interestingly, experiments in field stimulated cardiomyocytes with an increase in endogenous NO levels revealed PLM phosphorylation and increasing NKA activity to be independent of the cGMP/sGC/PKG pathway.

PLM is described to be functionally quiescent in terms of regulating intracellular $\mathrm{Ca}^{2+}$ and $\mathrm{Na}^{+}$ levels ${ }^{170}$, and hence cardiac contractility at basal state. Rather it is recognized as a cardiac stress protein under conditions of fight-or-flight response, when it enhances NKA activity to minimize the risk of arrhythmogenesis but inhibits NCX to preserve inotropy during stress ${ }^{173}$. Nevertheless, our experiments regarding the control of PDEs over basal cAMP in the vicinity of PLM uncovered a possible role of PDE2 CAMP hydrolysis under normal physiological conditions. Indeed, further experiments are required to quantify PDE2 regulated NKA activity and PDE2 dependent basal phosphorylation rate of PLM for a complete awareness of the physiological regulation of NKA with potentially PDE2 modulated PKA targeting of PLM.

Several studies reported the prominent role of PDE4 in CAMP compartmentation in proximity to $\mathrm{Ca}^{2+}$ handling proteins important for cardiac contraction. PDE4 isoforms PDE4B and PDE4D regulate cardiac contractility and $\mathrm{Ca}^{2+}$ cycling ${ }^{106}$, furthermore, PDE4D3 was reported to act on PKA-mediated RyR2 phosphorylation ${ }^{108}$. Our findings suggest a trend towards an elevated basal PDE4 inhibition effect on the PLM associated CAMP levels but this was found to be not statistically different.

\section{2. 2 Locally confined $\beta_{2}$-AR mediates cAMP signaling pathway proximal to the PLM/NKA complex}

It is well known that $\beta_{1}-A R$ induce far-reaching cAMP signals, whereas $\beta_{2}-A R$ signals remain locally confined ${ }^{28}$. Indeed, we found that $\beta_{1}-A R$ responses in PLM-E1 transduced ARVMs to Iso induced stimulation were similar to signals detected in the bulk cytosol (Figure 16C). Likewise, PDE-dependent regulation of these signals in the microdomain was not significantly different from cytosolic $\beta_{1}$-AR cAMP signals which is in line with the far-reaching control of $\beta_{1}$-ARs. In contrast, highly compartment specific $\beta_{2}$-AR signals led to greater cAMP dependent signal change in the PLM/NKA complex. There is an experimental evidence that Cav3 selectively modulates the localization of $\beta_{2}$-AR and spatially compartmentalizes its signaling to the T-tubular membrane compartments ${ }^{146}$. In conjunction with this, NKA is 
compartmentalized by its $\beta$-SU in Cav3 rich domains ${ }^{266}$, also suggested by previous observations in co-immunostainings of the NKA- $\alpha$-SU organized within cardiac caveolin-rich microdomains ${ }^{267}$. Based on our findings, it can be reasoned that $\beta_{2}$-ARs phosphorylate PLM and build a local signalosome, which might also contain NKA and NCX in close proximity (see chapter 1. 2. 1). The signal transduction to the PLM/NKA complex is tightly coupled to the regulation of intracellular $\mathrm{Ca}^{2+}$ and cardiac contractility ${ }^{268}$. Furthermore, Mohler et al. report that $\mathrm{NKA}, \mathrm{NCX} 1$, and $\mathrm{InsP}_{3} \mathrm{R}$ are complexed with ankyrin-B, an adapter protein within a microdomain of cardiomyocyte T-tubules ${ }^{75}$. This subcelllular complex is demonstrated as an essential requirement for $\mathrm{Ca}^{2+}$ homeostasis and proper cardiac function. This is consistent with our assumption that $\beta_{2}$-AR signals, which originate in T-tubules, reach the PLM compartment having an impact on $\mathrm{Na}^{+}$and $\mathrm{Ca}^{2+}$ cycling in cardiomyocytes.

As many previous publications ${ }^{269}$, this study used cultivated cardiomyocytes expressing cytosolic and targeted FRET biosensors introduced by adenoviruses, which might cause some structural remodeling on t-tubular level ${ }^{270}$. Despite application of the adenoviral system onto control cells transduced with cytosolic E1-camps, there was still the risk in losing the proper structure of important cAMP compartments in the complex intracellular architecture of cardiomyocytes during cultivation. However, recent studies using morphological analysis of cell membrane morphology in rat myocytes after $48 \mathrm{~h}$ of adenoviral transduction in culture under similar conditions could demonstrate the well-preserved integrity of sarcolemmal Ttubules $^{29}$. These morphological findings are supported by our experiments. Obtaining clearly distinct results for locally confined $\beta_{2}$-AR signals in the microdomain formed by PLM, we could confirm different organization of cytosolic and local signaling complex at the subcellular level.

\section{2. 3 Critical PDE3-dependent regulation of $\beta_{2}$-AR mediated cAMP in the vicinity of}

\section{PLM}

Next to their differential coupling to $G_{s}$ and $G_{i}$ proteins, the $\beta$-ARs differ in subtype specific cAMP signaling pathways and phosphorylation of substrates by local pools of PKA. This phenomenon is justified mainly through compartmentation of signaling events, such as the formation of signalosomes by AKAPs and PDEs (see chapter 1. 1. 2). Nikolaev et al. demonstrated that $\beta_{1}$-AR-mediated cytoplasmic CAMP is mainly controlled by PDE4, whereas cAMP produced after $\beta_{2}$-AR stimulation is under the control of multiple PDE isoforms ${ }^{28}$. $\beta_{2^{-}}$ ARs have a number of interacting proteins including different isoforms of PDE4D, whereas PDE4D8 has been shown to be associated with $\beta_{1}-A R s^{271}$. Recently, it was demonstrated that membrane localized $\beta_{2}$-AR associated microdomains in caveolin-rich membrane compartments are primarily modulated by PDE ${ }^{251}$. Similarly, in our FRET experiments we obtained increased $\beta_{2}$-CAMP sensing under Iso+CGP stimulated PLM-E1 transduced 
cardiomyocytes by inhibiting PDE3 (Figure 30F). PDE3 and PDE4 show equal contributions to the control of $\beta_{2}$-AR mediated cAMP signals in the cytosol (Figure 14D). Since the PLM-E1 biosensor can relatively well resolve $\beta_{2}$-signals in the microdomain (Figure12), it revealed the strong cAMP augmenting effect of PDE3 selective inhibitor cilostamide. This clearly suggested a strong PDE3 contribution in the signaling effect of $\beta_{2}$-CAMP directly at the site of the PLM/NKA complex. Preventing cAMP diffusion from $\beta_{2}-A R$ into the cytosol, PDE3 might act as a scaffolding enzyme to localize generated CAMP to the PLM compartment with its downstream targets, such as PKA. Even though some groups characterized $\beta_{2}$-ARs signals to be in tight control of PDE4D isoenzymes possibly organized by arrestins ${ }^{110,272}$, we and others $^{251}$ have proven that PDE3 might also play a significant role in $\beta_{2}$-AR associated microdomains, such as the PLM/NKA complex. Experiments demonstrate that cGMP inhibited PDE3 provides a sustained increase in CAMP under ANP stimulation, which can be abolished by pretreatment with the PDE3 selective inhibitor cilostamide (Figure 29). Physical proof of microdomain specific PDE3 activity was provided by co-localization analysis with coimmunostained freshly isolated WT-ARVMs. Here PDE3 showed a high co-localization with end. PLM (Figure 28). It is intriguing to argue that PDE3 is primarily responsible for the local modulation of PKA phosphorylation of PLM via $\beta_{2}$-ARs. Indeed, through previous work it was shown that PDE3 isoenzymes work as scaffolding proteins in spatially confined microdomains. For example, PDE3A localizes at the SERCA2 and SR-membrane whereby it tightly controls basal cardiac contractility by regulating CAMP mediated phosphorylation of PLB and sarcoplasmatic $\mathrm{Ca}^{2+}$ uptake through the SERCA2 $2^{273}$. As PDE3 is recognized to have a largely interacting role in cardiac $\mathrm{Ca}^{2+}$ cycling and serves as a clinical cardiotonic target to regulate $\beta$-AR inotropic responses in the heart ${ }^{274,275}$, it is likely that its compartmentalized regulation of PLM under neurohormonal stimulation of $\beta_{2}$-ARs is coupled to $\mathrm{Ca}^{2+}$-regulatory proteins residing in the same cardiac microdomain, i.e. the NCX.

The important finding in this work is the predominant activity of the PDE3 enzyme in the $\beta_{2^{-}}$ AR-cAMP pool in the vicinity of PLM, where cGMP can act here as a competitive inhibitor of PDE3 (Figure 16C). For that reason functional NO-GC activity and effective cGMP synthesis might be essential within this microdomain, which in turn appears to be myocyte and species specific in previous findings ${ }^{276}$. In case of the L-Type $\mathrm{Ca}^{2+}$ current, it was shown that its regulation via cGMP dependent PDE2/3 vary in different species and source of myocytes $^{263,277}$. Similar conditions prevailed in FRET experiments with aged matched control (AMC) animals used in this Ph.D. thesis, which in contrast did not reveal dominant PDE3 control over $\beta_{2}-A R$, rather generally comparable PDE profiles with the PLM microdomain were detected. The discrepancy between our findings in 8-12 weeks old Wistar rats and 24 weeks old Sprague Dawley aged matched control (AMC) animals regarding PDE responses to $\beta_{2}$-AR could be caused by age dependent alterations of the cardiac physiology, the race 
difference or this could be another example of local cGMP dependent PDE2/PDE3 crosstalk. Despite being not significantly different $(p=0.13)$, there is indeed a clear trend of local PDE2 to respond to selective inhibition upon $\beta_{2}-A R$ stimulation in Sprague Dawley AMC animals. So there is an appreciated level of potentially cGMP activated PDE2 pool in the $\beta_{2}$-AR controlled PLM microdomain in 24 weeks old rats. In this regard, further experiments in these animals are necessary, which could better explain the moderate FRET change in local PDE3 response compared to Wistar rats (i.e. different NO-GC-cGMP levels, age related and race dependent differences in cardiac physiology, differences of genetic background).

In conclusion, one could postulate that there is a locally confined cAMP effect on PLM regulated by potentially cGMP dependent PDE3, which specifically hydrolyzes the local pool of $\beta_{2}$-AR-CAMP thereby forming a microdomain and disabling diffusion of the second messenger into the cytosol. In addition to its effects on the NKA and NCX (see chapter 1. 2. 1) there is a possibility that in cardiomyocytes, PLM might potentially be involved in the kinetics of $\mathrm{LTCCs}^{207}$. Under these conditions our findings about the role PDE3 in the PLMNKA microdomain might have a higher complexity in terms of cardiac contractility. As stated above, PDE3 inhibiting cardiotonic compounds are used in the clinic and could also induce a local increase in $\beta_{2}$-cAMP and subsequent PKA phosphorylation of PLM resulting in higher sodium and calcium efflux. The enhanced lusitropic effects would protect from $\mathrm{Na}^{+}$and $\mathrm{Ca}^{2+}$ overload in hypertrophied hearts and limit risks associated with NKA inhibition by cardiac glycosides, such as triggered arrhythmias.

\section{3. Alterations of $\beta_{2}$-AR control over subsarcolemmal PLM microdomain through localized PDE2/PDE3 subsets in ARVM with chronic heart failure}

FRET measurements in ARVMs from animals 16 weeks after myocardial infarction showed that the significant differences in $\beta_{2}$-AR stimulation between the bulk cytosol and the PLM/NKA microdomain were abolished (Figure 31 and Figure 32). This loss of communication between $\beta_{2}$-AR and the PLM microdomain is in line with pathophysiological changes occurring during transitioning from early cardiac disease to the functionally decompensated state of the heart, which comes along with structural deformation of subcellular systems and global as well as local alterations in signaling complexes important for proper cardiac function (Figure 5C). Especially, alterations in PDE effects are recognized in hypertrophied cells. Increasing body of evidence demonstrate a clear increase in whole cell PDE2 activity at end stage of heart failure ${ }^{155,278}$ protecting the diseased heart from stress, which is caused by excessive catecholamine dumping at this state. Also in early stage of HF, it was shown that locally detected PDE2 effects at the $\beta_{2}$-AR were changed, while whole cell PDE2 amounts and activities remained stable ${ }^{251}$. This microdomain specific replacement of cGMP inhibited PDE3 with cGMP activated PDE2 was postulated to be a positive adaptive 
mechanism to increased ANP/cGMP effects in hypertrophy. Similarly, our FRET experiments in PLM-E1 transduced MI cardiomyocytes revealed a significant decrease of PDE3 response after $\beta_{2}$-AR stimulation whereas PDE2 contributions increased in the PLM/NKA microdomain. Likewise, PDE3 and PLM co-immunostained MI cells showed a significantly decreased colocalization pattern compared to AMC cells (Figure 32). Interestingly, after $\beta_{2}$-AR stimulation no differences between AMC and MI cells in PDE contributions to cAMP hydrolysis in the bulk cytosol were detected. This might indicate that there is no change in global PDE activity after congestive heart failure in ARVMs, but rather subcellular alterations in PDE catalytic activity. Previously, it was shown that in rat hearts post-MI, the NKA expression and activity were depressed, although the determined overexpression of PLM in Ml cells may also account for reduced NKA activity ${ }^{173}$ and altered cardiac contractility ${ }^{279}$. In addition to previous findings, we detected a change in local $\beta_{2}-A R$ signals in Ml myocytes and a decrease in PDE3 controlled $\beta_{2}$-CAMP in the PLM microdomain being substituted by an increasing PDE2 effect. Our findings suggest impaired local signaling in the $\beta_{2}$-PLM-NKA network which may contribute to contractile and electrical dysfunction in arrhythmia upon ischemic disease. Since the implemented disease model for this Ph.D. thesis is an example of decompensated hypertrophy and chronic heart failure, further investigations about sarcolemmal architecture, altered $\beta$-AR densities and PLM localized changes of PDE effects on $\beta_{1}$-AR-cAMP would serve for a more detailed characterization of adrenergic signaling cascade between the receptor and PLM microdomain in cardiac disease. 


\section{Conclusion and Outlook}

The PLM/NKA complex provides the only significant cardiac $\mathrm{Na}^{+}$efflux pathway and is therefore vital for normal physiological function and an important target for therapy during congestive heart failure. The aim of this thesis was to extensively study real time dynamics of CAMP in the PLM-associated microdomain controlled by $\beta$-adrenergic signaling and its alterations in chronic heart disease. In order to determine differential regulation of the PLM microdomain via subtype specific $\beta$-AR signaling and changes of PDE effects over local pools of CAMP, a new localized FRET based CAMP sensitive biosensor named PLM-E1 was successfully generated und characterized. For the FRET based experiments an adenoviral system was used, which enabled titer controlled measurements in transduced cardiomyocytes after $48 \mathrm{~h}$ cultivation. The present study confirmed the existence of a differentially regulated PLM/NKA compartment confined from the cytosolic domain through individual PDE subtype contributions to the cAMP degradation under selective stimulation of $\beta$-AR subtypes. It identified a PDE2 dependent local cAMP regulation in the vicinity of PLM (Figure 33), which could be further explored in subsequent biochemical or electrophysiological studies, giving insights about PDE2 controlled PLM phosphorylation and NKA function or both. The second essential finding is that there is a tight coupling of $\beta_{2}-A R$ signals to the PLM/NKA complex regulated by a specific PDE pattern, which is changed dramatically in post-infarcted failing cardiomyocytes (Figure 34). We provided some interesting insights but in terms of PDE regulated CAMP/PKA signaling to PLM it would be exciting to investigate sodium and calcium induced local changes in cAMP at the plasma membrane. Considering its importance in cardiac ECC and the complex role of PLM controlling NKA, NCX or LTCCs ${ }^{207}$ by having three different substrate residues for PKA and PKC, it would be intriguing to investigate kinase activities and cGMP dynamics via PKC and cGMP sensitive FRET based localized biosensors.

In the longer term, our observations of PDE-dependent cAMP dynamics at the PLM/NKA complex and their changes in heart failure may serve not only as a basis for further studies but may also be interesting for clinical treatment of impaired NKA activity leading to sodium and calcium overload. Particularly, selective targeting of PDE2 could be a therapeutic approach during cardiac arrhythmia or increased catecholaminergic stress which may lead to low intracellular sodium levels by elevating the sodium pump activity. 


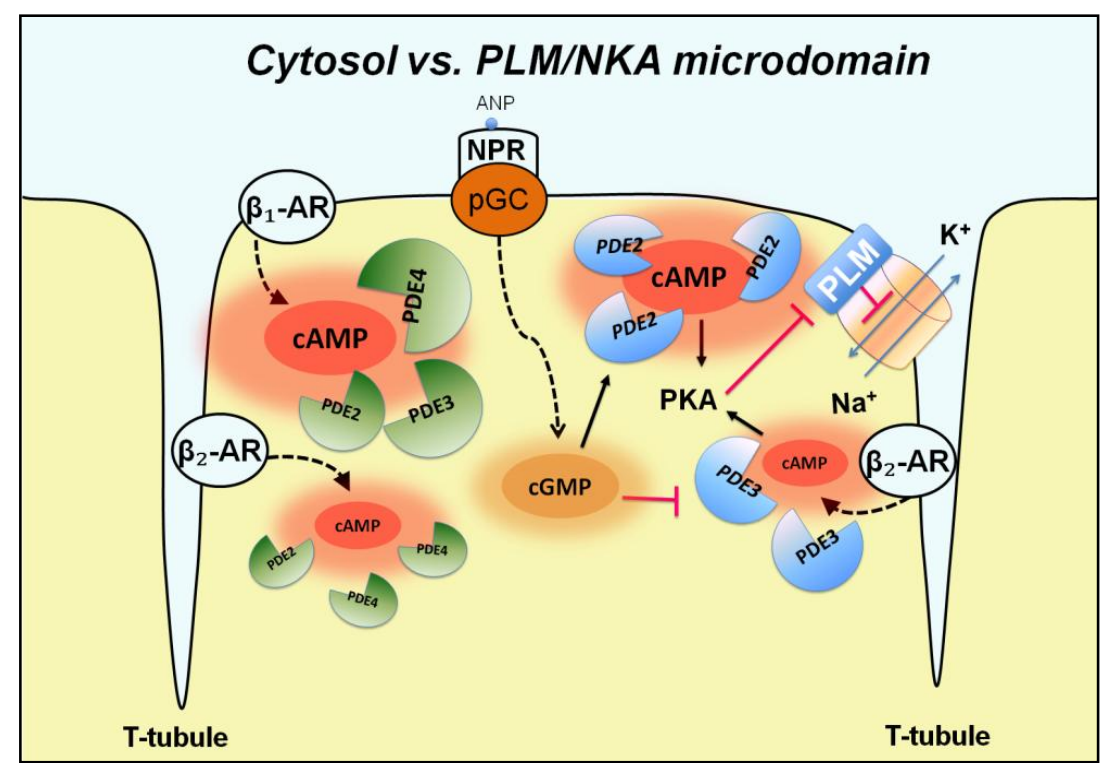

Figure 33. Cartoon showing highly confined basal cAMP dynamics and a distinct $\beta_{2}$-cAMP effect in the PLM microdomain of ARVMs. There is a predominant PDE2 control of the basal CAMP pool in the vicinity of PLM, whilst the microdomain specific PDE activities under $\beta_{1}$-AR stimulation are comparable to those in the cytosol (PDE4>PDE3>>PDE2). Far reaching $\beta_{1}$-AR signals equally effect the PLM/NKA complex. The $\beta_{2}-A R$ mediated cAMP effects on PLM are mainly controlled via cGMP dependent PDE3, while the low cytosolic cAMP amounts are hydrolyzed uniformly by PDE2-4. The cAMP/PKA regulation of the $\beta_{2}-A R$ associated PLM/NKA complex strongly rely on PDE enzymes activated (PDE2) and inhibited (PDE3) by cGMP.
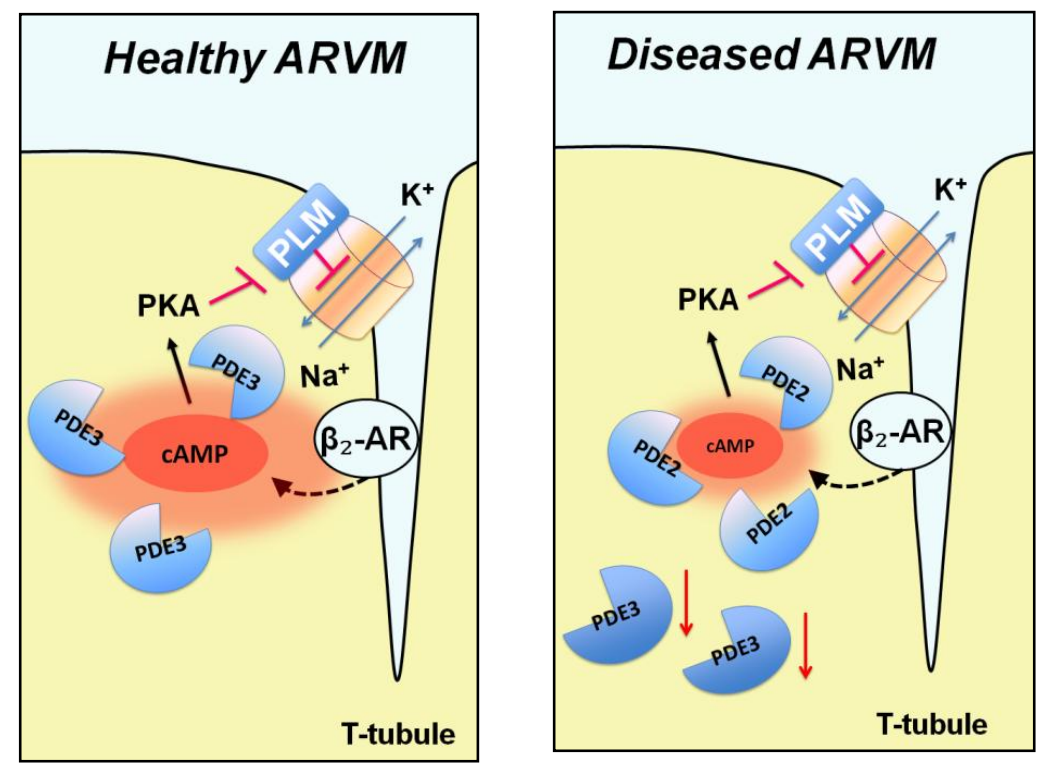

Figure 34. Cartoon demonstrating the change in local cAMP dynamics at the cardiac PLM microdomain. In healthy ARVMs there is a privileged communication between $\beta_{2}-A R$ and PLM/NKA microdomain, which is altered in chronic heart failure. This includes the general decrease in the $\beta_{2}-A R$ mediated cAMP effect and the substitution of its former PDE3 regulation with PDE2 at the $\beta_{2}-A R$ linked PLM/NKA complex. 


\section{$\underline{\text { 6. Bibliography }}$}

1 Lafontan, M. et al. Adrenergic regulation of adipocyte metabolism. Human reproduction 12 Suppl 1, 6-20 (1997).

2 Brudvik, K. W. \& Tasken, K. Modulation of T cell immune functions by the prostaglandin E(2) CAMP pathway in chronic inflammatory states. British journal of pharmacology 166, 411-419, doi:10.1111/j.1476-5381.2011.01800.x (2012).

3 Nishimura, K., Tamaoki, J., Isono, K., Aoshiba, K. \& Nagai, A. Beta-adrenergic receptormediated growth of human airway epithelial cell lines. The European respiratory journal 20, 353-358 (2002).

4 Morgado, M., Cairrao, E., Santos-Silva, A. J. \& Verde, I. Cyclic nucleotide-dependent relaxation pathways in vascular smooth muscle. Cellular and molecular life sciences : CMLS 69, 247-266, doi:10.1007/s00018-011-0815-2 (2012). Iwai-Kanai, E. et al. alpha- and beta-adrenergic pathways differentially regulate cell typespecific apoptosis in rat cardiac myocytes. Circulation 100, 305-311 (1999). Gordan, R., Gwathmey, J. K. \& Xie, L. H. Autonomic and endocrine control of cardiovascular function. World journal of cardiology 7, 204-214, doi:10.4330/wjc.v7.i4.204 (2015). Madamanchi, A. Beta-adrenergic receptor signaling in cardiac function and heart failure. McGill journal of medicine : MJM : an international forum for the advancement of medical sciences by students 10, 99-104 (2007).

8 Taylor, S. S., Buechler, J. A. \& Yonemoto, W. cAMP-dependent protein kinase: framework for a diverse family of regulatory enzymes. Annual review of biochemistry 59, 971-1005, doi:10.1146/annurev.bi.59.070190.004543 (1990).

9 Biel, M. \& Michalakis, S. Cyclic nucleotide-gated channels. Handbook of experimental pharmacology, 111-136, doi:10.1007/978-3-540-68964-5_7 (2009).

10 Wright, P. T., Schobesberger, S. \& Gorelik, J. Studying GPCR/cAMP pharmacology from the perspective of cellular structure. Frontiers in pharmacology 6, 148, doi:10.3389/fphar.2015.00148 (2015).

11 de Rooij, J. et al. Epac is a Rap1 guanine-nucleotide-exchange factor directly activated by cyclic AMP. Nature 396, 474-477, doi:10.1038/24884 (1998).

12 Metrich, M. et al. Epac mediates beta-adrenergic receptor-induced cardiomyocyte hypertrophy. Circulation research 102, 959-965, doi:10.1161/CIRCRESAHA.107.164947 (2008).

13 Krebs, E. G. \& Beavo, J. A. Phosphorylation-dephosphorylation of enzymes. Annual review of biochemistry 48, 923-959, doi:10.1146/annurev.bi.48.070179.004423 (1979).

14 Lohse, M. J., Engelhardt, S. \& Eschenhagen, T. What is the role of beta-adrenergic signaling in heart failure? Circulation research 93, 896-906, doi:10.1161/01.RES.0000102042.83024.CA (2003).

15 Despa, S. et al. Phospholemman-phosphorylation mediates the beta-adrenergic effects on $\mathrm{Na} / \mathrm{K}$ pump function in cardiac myocytes. Circulation research $97,252-259$, doi:10.1161/01.RES.0000176532.97731.e5 (2005).

16 Bers, D. M. Cardiac excitation-contraction coupling. Nature 415, 198-205, doi:10.1038/415198a (2002).

17 Bers, D. M. \& Despa, S. Na/K-ATPase--an integral player in the adrenergic fight-or-flight response. Trends in cardiovascular medicine 19, 111-118, doi:10.1016/j.tcm.2009.07.001 (2009).

18 Grimm, M. \& Brown, J. H. Beta-adrenergic receptor signaling in the heart: role of CaMKII. Journal of molecular and cellular cardiology 48, 322-330, doi:10.1016/j.yjmcc.2009.10.016 (2010).

19 Abraham, G., Kneuer, C., Ehrhardt, C., Honscha, W. \& Ungemach, F. R. Expression of functional beta2-adrenergic receptors in the lung epithelial cell lines $16 \mathrm{HBE} 14 \mathrm{o}(-)$, Calu-3 and 
A549. Biochimica et biophysica acta 1691, 169-179, doi:10.1016/j.bbamcr.2004.02.002 (2004).

Healy, D. P., Munzel, P. A. \& Insel, P. A. Localization of beta 1- and beta 2-adrenergic receptors in rat kidney by autoradiography. Circulation research 57, 278-284 (1985).

Zhou, Y. Y. et al. Localized cAMP-dependent signaling mediates beta 2-adrenergic modulation of cardiac excitation-contraction coupling. The American journal of physiology 273, H16111618 (1997).

22 Xiao, R. P., Ji, X. \& Lakatta, E. G. Functional coupling of the beta 2-adrenoceptor to a pertussis toxin-sensitive $G$ protein in cardiac myocytes. Molecular pharmacology 47, 322-329 (1995). Kuschel, M. et al. beta2-adrenergic cAMP signaling is uncoupled from phosphorylation of cytoplasmic proteins in canine heart. Circulation 99, 2458-2465 (1999).

24 Altschuld, R. A. et al. Response of failing canine and human heart cells to beta 2-adrenergic stimulation. Circulation 92, 1612-1618 (1995).

25 Levy, F. O., Zhu, X., Kaumann, A. J. \& Birnbaumer, L. Efficacy of beta 1-adrenergic receptors is lower than that of beta 2-adrenergic receptors. Proceedings of the National Academy of Sciences of the United States of America 90, 10798-10802 (1993).

26 Bristow, M. R., Hershberger, R. E., Port, J. D., Minobe, W. \& Rasmussen, R. Beta 1- and beta 2-adrenergic receptor-mediated adenylate cyclase stimulation in nonfailing and failing human ventricular myocardium. Molecular pharmacology 35, 295-303 (1989). Green, S. A., Holt, B. D. \& Liggett, S. B. Beta 1- and beta 2-adrenergic receptors display subtype-selective coupling to Gs. Molecular pharmacology 41, 889-893 (1992). Nikolaev, V. O., Bunemann, M., Schmitteckert, E., Lohse, M. J. \& Engelhardt, S. Cyclic AMP imaging in adult cardiac myocytes reveals far-reaching beta1-adrenergic but locally confined beta2-adrenergic receptor-mediated signaling. Circulation research 99, 1084-1091, doi:10.1161/01.RES.0000250046.69918.d5 (2006).

29 Nikolaev, V. O. et al. Beta2-adrenergic receptor redistribution in heart failure changes cAMP compartmentation. Science 327, 1653-1657, doi:10.1126/science.1185988 (2010).

Chen-Izu, Y. et al. G(i)-dependent localization of beta(2)-adrenergic receptor signaling to Ltype $\mathrm{Ca}(2+)$ channels. Biophysical journal 79, 2547-2556, doi:10.1016/S0006-3495(00)764952 (2000).

31 Zheng, M., Han, Q. D. \& Xiao, R. P. Distinct beta-adrenergic receptor subtype signaling in the heart and their pathophysiological relevance. Sheng li xue bao : [Acta physiologica Sinica] 56, 1-15 (2004).

32 Zhu, W. Z. et al. Dual modulation of cell survival and cell death by beta(2)-adrenergic signaling in adult mouse cardiac myocytes. Proceedings of the National Academy of Sciences of the United States of America 98, 1607-1612, doi:10.1073/pnas.98.4.1607 (2001).

33 Cannavo, A., Liccardo, D. \& Koch, W. J. Targeting cardiac beta-adrenergic signaling via GRK2 inhibition for heart failure therapy. Frontiers in physiology 4, 264, doi:10.3389/fphys.2013.00264 (2013).

34 Lohse, M. J., Benovic, J. L., Codina, J., Caron, M. G. \& Lefkowitz, R. J. beta-Arrestin: a protein that regulates beta-adrenergic receptor function. Science 248, 1547-1550 (1990).

35 Bristow, M. R. et al. Beta 1- and beta 2-adrenergic-receptor subpopulations in nonfailing and failing human ventricular myocardium: coupling of both receptor subtypes to muscle contraction and selective beta 1-receptor down-regulation in heart failure. Circulation research 59, 297-309 (1986).

36 Brodde, O. E. Beta-adrenoceptors in cardiac disease. Pharmacology \& therapeutics 60, 405430 (1993).

37 Bristow, M. R. et al. Decreased catecholamine sensitivity and beta-adrenergic-receptor density in failing human hearts. The New England journal of medicine 307, 205-211, doi:10.1056/NEJM198207223070401 (1982). 

beta(2)-adrenergic receptors on cardiac myocyte apoptosis : role of a pertussis toxinsensitive $\mathrm{G}$ protein. Circulation 100, 2210-2212 (1999). Brodde, O. E. The functional importance of beta 1 and beta 2 adrenoceptors in the human heart. The American journal of cardiology 62, 24C-29C (1988). Krief, S. et al. Tissue distribution of beta 3-adrenergic receptor mRNA in man. The Journal of clinical investigation 91, 344-349, doi:10.1172/JCI116191 (1993).

41 Devic, E., Xiang, Y., Gould, D. \& Kobilka, B. Beta-adrenergic receptor subtype-specific signaling in cardiac myocytes from beta(1) and beta(2) adrenoceptor knockout mice. Molecular pharmacology 60, 577-583 (2001).

42 Kohout, T. A. et al. Augmentation of cardiac contractility mediated by the human beta(3)adrenergic receptor overexpressed in the hearts of transgenic mice. Circulation 104, 24852491 (2001).

43 Gerhardt, C. C., Gros, J., Strosberg, A. D. \& Issad, T. Stimulation of the extracellular signalregulated kinase $1 / 2$ pathway by human beta-3 adrenergic receptor: new pharmacological profile and mechanism of activation. Molecular pharmacology 55, 255-262 (1999).

44 Soeder, K. J. et al. The beta3-adrenergic receptor activates mitogen-activated protein kinase in adipocytes through a Gi-dependent mechanism. The Journal of biological chemistry 274, 12017-12022 (1999).

45 Belge, C. et al. Enhanced expression of beta3-adrenoceptors in cardiac myocytes attenuates neurohormone-induced hypertrophic remodeling through nitric oxide synthase. Circulation 129, 451-462, doi:10.1161/CIRCULATIONAHA.113.004940 (2014).

46 Hammond, J. \& Balligand, J. L. Nitric oxide synthase and cyclic GMP signaling in cardiac myocytes: from contractility to remodeling. Journal of molecular and cellular cardiology 52, 330-340, doi:10.1016/j.yjmcc.2011.07.029 (2012).

47 Berthet, J., Rall, T. W. \& Sutherland, E. W. The relationship of epinephrine and glucagon to liver phosphorylase. IV. Effect of epinephrine and glucagon on the reactivation of phosphorylase in liver homogenates. The Journal of biological chemistry 224, 463-475 (1957).

48 Kandel, E. R. The molecular biology of memory: CAMP, PKA, CRE, CREB-1, CREB-2, and CPEB. Molecular brain 5, 14, doi:10.1186/1756-6606-5-14 (2012).

49 Shi, W. X. \& Bunney, B. S. Roles of intracellular cAMP and protein kinase $A$ in the actions of dopamine and neurotensin on midbrain dopamine neurons. The Journal of neuroscience : the official journal of the Society for Neuroscience 12, 2433-2438 (1992).

50 Yajima, H. et al. cAMP enhances insulin secretion by an action on the ATP-sensitive K+ channel-independent pathway of glucose signaling in rat pancreatic islets. Diabetes $\mathbf{4 8 , 1 0 0 6 -}$ 1012 (1999).

51 Tengholm, A. \& Gylfe, E. Oscillatory control of insulin secretion. Molecular and cellular endocrinology 297, 58-72, doi:10.1016/j.mce.2008.07.009 (2009).

52 Mayr, B. M., Canettieri, G. \& Montminy, M. R. Distinct effects of cAMP and mitogenic signals on CREB-binding protein recruitment impart specificity to target gene activation via CREB. Proceedings of the National Academy of Sciences of the United States of America 98, 1093610941, doi:10.1073/pnas.191152098 (2001).

53 Altarejos, J. Y. \& Montminy, M. CREB and the CRTC co-activators: sensors for hormonal and metabolic signals. Nature reviews. Molecular cell biology 12, 141-151, doi:10.1038/nrm3072 (2011).

54 Bodor, J. et al. Cyclic AMP underpins suppression by regulatory T cells. European journal of immunology 42, 1375-1384, doi:10.1002/eji.201141578 (2012).

55 Zagotta, W. N. et al. Structural basis for modulation and agonist specificity of HCN pacemaker channels. Nature 425, 200-205, doi:10.1038/nature01922 (2003).

56 Bai, Y. \& Sanderson, M. J. Airway smooth muscle relaxation results from a reduction in the frequency of $\mathrm{Ca} 2+$ oscillations induced by a cAMP-mediated inhibition of the IP3 receptor. Respiratory research 7, 34, doi:10.1186/1465-9921-7-34 (2006). 
Hayes, J. S., Brunton, L. L., Brown, J. H., Reese, J. B. \& Mayer, S. E. Hormonally specific expression of cardiac protein kinase activity. Proceedings of the National Academy of Sciences of the United States of America 76, 1570-1574 (1979). Vila Petroff, M. G., Egan, J. M., Wang, X. \& Sollott, S. J. Glucagon-like peptide-1 increases cAMP but fails to augment contraction in adult rat cardiac myocytes. Circulation research 89 , 445-452 (2001).

59 Hohl, C. M. \& Li, Q. A. Compartmentation of cAMP in adult canine ventricular myocytes. Relation to single-cell free Ca2+ transients. Circulation research 69, 1369-1379 (1991). Xiao, R. P. et al. Beta 2-adrenergic receptor-stimulated increase in cAMP in rat heart cells is not coupled to changes in $\mathrm{Ca} 2+$ dynamics, contractility, or phospholamban phosphorylation. The Journal of biological chemistry 269, 19151-19156 (1994).

61 Di Benedetto, G. et al. Protein kinase A type I and type II define distinct intracellular signaling compartments. Circulation research 103, 836-844, doi:10.1161/CIRCRESAHA.108.174813 (2008).

62 Zaccolo, M. cAMP signal transduction in the heart: understanding spatial control for the development of novel therapeutic strategies. British journal of pharmacology 158, 50-60, doi:10.1111/j.1476-5381.2009.00185.x (2009).

63 Lefkimmiatis, K., Leronni, D. \& Hofer, A. M. The inner and outer compartments of mitochondria are sites of distinct CAMP/PKA signaling dynamics. The Journal of cell biology 202, 453-462, doi:10.1083/jcb.201303159 (2013).

64 Sample, V. et al. Regulation of nuclear PKA revealed by spatiotemporal manipulation of cyclic AMP. Nature chemical biology 8, 375-382, doi:10.1038/nchembio.799 (2012).

65 Mongillo, M. et al. Fluorescence resonance energy transfer-based analysis of cAMP dynamics in live neonatal rat cardiac myocytes reveals distinct functions of compartmentalized phosphodiesterases. Circulation research 95, 67-75, doi:10.1161/01.RES.0000134629.84732.11 (2004).

66 DiPilato, L. M. \& Zhang, J. The role of membrane microdomains in shaping beta2-adrenergic receptor-mediated cAMP dynamics. Molecular bioSystems 5, 832-837, doi:10.1039/b823243a (2009).

67 Rich, T. C. et al. Cellular mechanisms underlying prostaglandin-induced transient cAMP signals near the plasma membrane of HEK-293 cells. American journal of physiology. Cell physiology 292, C319-331, doi:10.1152/ajpcell.00121.2006 (2007).

68 Agarwal, S. R. et al. Role of membrane microdomains in compartmentation of cAMP signaling. PloS one 9, e95835, doi:10.1371/journal.pone.0095835 (2014).

69 Pani, B. \& Singh, B. B. Lipid rafts/caveolae as microdomains of calcium signaling. Cell calcium 45, 625-633, doi:10.1016/j.ceca.2009.02.009 (2009).

70 Jurevicius, J. \& Fischmeister, R. cAMP compartmentation is responsible for a local activation of cardiac Ca2+ channels by beta-adrenergic agonists. Proceedings of the National Academy of Sciences of the United States of America 93, 295-299 (1996).

71 Davare, M. A. et al. A beta2 adrenergic receptor signaling complex assembled with the $\mathrm{Ca} 2+$ channel Cav1.2. Science 293, 98-101, doi:10.1126/science.293.5527.98 (2001).

72 Zaccolo, M. \& Pozzan, T. Discrete microdomains with high concentration of cAMP in stimulated rat neonatal cardiac myocytes. Science 295, 1711-1715, doi:10.1126/science.1069982 (2002).

73 Hulme, J. T., Lin, T. W., Westenbroek, R. E., Scheuer, T. \& Catterall, W. A. Beta-adrenergic regulation requires direct anchoring of PKA to cardiac CaV1.2 channels via a leucine zipper interaction with A kinase-anchoring protein 15. Proceedings of the National Academy of Sciences of the United States of America 100, 13093-13098, doi:10.1073/pnas.2135335100 (2003).

74 Hofmann, F., Flockerzi, V., Kahl, S. \& Wegener, J. W. L-type CaV1.2 calcium channels: from in vitro findings to in vivo function. Physiological reviews 94, 303-326, doi:10.1152/physrev.00016.2013 (2014). 
75 Mohler, P. J., Davis, J. Q. \& Bennett, V. Ankyrin-B coordinates the Na/K ATPase, Na/Ca exchanger, and InsP3 receptor in a cardiac T-tubule/SR microdomain. PLoS biology 3, e423, doi:10.1371/journal.pbio.0030423 (2005).

76 Crambert, G., Fuzesi, M., Garty, H., Karlish, S. \& Geering, K. Phospholemman (FXYD1) associates with $\mathrm{Na}$,K-ATPase and regulates its transport properties. Proceedings of the National Academy of Sciences of the United States of America 99, 11476-11481, doi:10.1073/pnas.182267299 (2002).

77 Brette, F., Salle, L. \& Orchard, C. H. Quantification of calcium entry at the T-tubules and surface membrane in rat ventricular myocytes. Biophysical journal 90, 381-389, doi:10.1529/biophysj.105.069013 (2006).

78 Brette, F. \& Orchard, C. T-tubule function in mammalian cardiac myocytes. Circulation research 92, 1182-1192, doi:10.1161/01.RES.0000074908.17214.FD (2003).

79 Perera, R. K. \& Nikolaev, V. O. Compartmentation of cAMP signalling in cardiomyocytes in health and disease. Acta physiologica 207, 650-662, doi:10.1111/apha.12077 (2013).

80 Conti, M. \& Beavo, J. Biochemistry and physiology of cyclic nucleotide phosphodiesterases: essential components in cyclic nucleotide signaling. Annual review of biochemistry 76, 481511, doi:10.1146/annurev.biochem.76.060305.150444 (2007).

81 Francis, S. H., Blount, M. A. \& Corbin, J. D. Mammalian cyclic nucleotide phosphodiesterases: molecular mechanisms and physiological functions. Physiological reviews 91, 651-690, doi:10.1152/physrev.00030.2010 (2011).

82 Lugnier, C. Cyclic nucleotide phosphodiesterase (PDE) superfamily: a new target for the development of specific therapeutic agents. Pharmacology \& therapeutics 109, 366-398, doi:10.1016/j.pharmthera.2005.07.003 (2006).

83 Zhang, M. \& Kass, D. A. Phosphodiesterases and cardiac cGMP: evolving roles and controversies. Trends in pharmacological sciences 32, 360-365, doi:10.1016/j.tips.2011.02.019 (2011).

84 Takimoto, E. Cyclic GMP-dependent signaling in cardiac myocytes. Circulation journal : official journal of the Japanese Circulation Society 76, 1819-1825 (2012).

85 Nikolaev, V. O. \& Lohse, M. J. Monitoring of cAMP synthesis and degradation in living cells. Physiology 21, 86-92, doi:10.1152/physiol.00057.2005 (2006).

86 Sonnenburg, W. K., Seger, D. \& Beavo, J. A. Molecular cloning of a cDNA encoding the "61kDa" calmodulin-stimulated cyclic nucleotide phosphodiesterase. Tissue-specific expression of structurally related isoforms. The Journal of biological chemistry 268, 645-652 (1993).

87 Miller, C. L. \& Yan, C. Targeting cyclic nucleotide phosphodiesterase in the heart: therapeutic implications. Journal of cardiovascular translational research 3, 507-515, doi:10.1007/s12265-010-9203-9 (2010).

88 Vandeput, F. et al. Cyclic nucleotide phosphodiesterase PDE1C1 in human cardiac myocytes. The Journal of biological chemistry 282, 32749-32757, doi:10.1074/jbc.M703173200 (2007).

89 Miller, C. L. et al. Role of Ca2+/calmodulin-stimulated cyclic nucleotide phosphodiesterase 1 in mediating cardiomyocyte hypertrophy. Circulation research 105, 956-964, doi:10.1161/CIRCRESAHA.109.198515 (2009).

90 Martinez, S. E. et al. The two GAF domains in phosphodiesterase 2A have distinct roles in dimerization and in CGMP binding. Proceedings of the National Academy of Sciences of the United States of America 99, 13260-13265, doi:10.1073/pnas.192374899 (2002).

91 Mongillo, M. et al. Compartmentalized phosphodiesterase-2 activity blunts beta-adrenergic cardiac inotropy via an NO/cGMP-dependent pathway. Circulation research 98, 226-234, doi:10.1161/01.RES.0000200178.34179.93 (2006).

92 Muller, B., Stoclet, J. C. \& Lugnier, C. Cytosolic and membrane-bound cyclic nucleotide phosphodiesterases from guinea pig cardiac ventricles. European journal of pharmacology 225, 263-272 (1992). 
93 Castro, L. R., Verde, I., Cooper, D. M. \& Fischmeister, R. Cyclic guanosine monophosphate compartmentation in rat cardiac myocytes. Circulation 113, 2221-2228, doi:10.1161/CIRCULATIONAHA.105.599241 (2006).

94 Stangherlin, A. et al. cGMP signals modulate cAMP levels in a compartment-specific manner to regulate catecholamine-dependent signaling in cardiac myocytes. Circulation research 108, 929-939, doi:10.1161/CIRCRESAHA.110.230698 (2011). Wechsler, J. et al. Isoforms of cyclic nucleotide phosphodiesterase PDE3A in cardiac myocytes. The Journal of biological chemistry 277, 38072-38078, doi:10.1074/jbc.M203647200 (2002).

96 Shakur, Y. et al. Regulation and function of the cyclic nucleotide phosphodiesterase (PDE3) gene family. Progress in nucleic acid research and molecular biology 66, 241-277 (2001).

97 Sun, B. et al. Role of phosphodiesterase type 3A and 3B in regulating platelet and cardiac function using subtype-selective knockout mice. Cellular signalling 19, 1765-1771, doi:10.1016/j.cellsig.2007.03.012 (2007).

98 Hambleton, R. et al. Isoforms of cyclic nucleotide phosphodiesterase PDE3 and their contribution to CAMP hydrolytic activity in subcellular fractions of human myocardium. The Journal of biological chemistry 280, 39168-39174, doi:10.1074/jbc.M506760200 (2005). Murashima, S., Tanaka, T., Hockman, S. \& Manganiello, V. Characterization of particulate cyclic nucleotide phosphodiesterases from bovine brain: purification of a distinct cGMPstimulated isoenzyme. Biochemistry 29, 5285-5292 (1990).

100 Shakur, Y. et al. Membrane localization of cyclic nucleotide phosphodiesterase 3 (PDE3). Two $\mathrm{N}$-terminal domains are required for the efficient targeting to, and association of, PDE3 with endoplasmic reticulum. The Journal of biological chemistry 275, 38749-38761, doi:10.1074/jbc.M001734200 (2000).

101 Degerman, E., Belfrage, P. \& Manganiello, V. C. Structure, localization, and regulation of cGMP-inhibited phosphodiesterase (PDE3). The Journal of biological chemistry 272, 68236826 (1997).

102 Abi-Gerges, A. et al. Decreased expression and activity of cAMP phosphodiesterases in cardiac hypertrophy and its impact on beta-adrenergic CAMP signals. Circulation research 105, 784-792, doi:10.1161/CIRCRESAHA.109.197947 (2009).

103 Fischmeister, R. et al. Compartmentation of cyclic nucleotide signaling in the heart: the role of cyclic nucleotide phosphodiesterases. Circulation research 99, 816-828, doi:10.1161/01.RES.0000246118.98832.04 (2006).

104 Richter, W., Jin, S. L. \& Conti, M. Splice variants of the cyclic nucleotide phosphodiesterase PDE4D are differentially expressed and regulated in rat tissue. The Biochemical journal 388, 803-811, doi:10.1042/BJ20050030 (2005).

105 Baillie, G. S. \& Houslay, M. D. Arrestin times for compartmentalised cAMP signalling and phosphodiesterase-4 enzymes. Current opinion in cell biology 17, 129-134, doi:10.1016/j.ceb.2005.01.003 (2005).

106 Leroy, J. et al. Phosphodiesterase 4B in the cardiac L-type $\mathrm{Ca}(2)(+)$ channel complex regulates $\mathrm{Ca}(2)(+)$ current and protects against ventricular arrhythmias in mice. The Journal of clinical investigation 121, 2651-2661, doi:10.1172/JCl44747 (2011).

107 Dodge, K. L. et al. mAKAP assembles a protein kinase A/PDE4 phosphodiesterase cAMP signaling module. The EMBO journal 20, 1921-1930, doi:10.1093/emboj/20.8.1921 (2001).

108 Lehnart, S. E. et al. Phosphodiesterase 4D deficiency in the ryanodine-receptor complex promotes heart failure and arrhythmias. Cell 123, 25-35, doi:10.1016/j.cell.2005.07.030 (2005).

109 Perry, S. J. et al. Targeting of cyclic AMP degradation to beta 2-adrenergic receptors by betaarrestins. Science 298, 834-836, doi:10.1126/science.1074683 (2002).

110 Richter, W. et al. Signaling from beta1- and beta2-adrenergic receptors is defined by differential interactions with PDE4. The EMBO journal 27, 384-393, doi:10.1038/sj.emboj.7601968 (2008). 
111 Ghigo, A. et al. Phosphoinositide 3-kinase gamma protects against catecholamine-induced ventricular arrhythmia through protein kinase A-mediated regulation of distinct phosphodiesterases. Circulation 126, 2073-2083, doi:10.1161/CIRCULATIONAHA.112.114074 (2012).

112 Lindman, B. R. et al. Effects of phosphodiesterase type 5 inhibition on systemic and pulmonary hemodynamics and ventricular function in patients with severe symptomatic aortic stenosis. Circulation 125, 2353-2362, doi:10.1161/CIRCULATIONAHA.111.081125 (2012).

113 Shan, X. et al. Differential expression of PDE5 in failing and nonfailing human myocardium. Circulation. Heart failure 5, 79-86, doi:10.1161/CIRCHEARTFAILURE.111.961706 (2012).

114 Lee, D. I. \& Kass, D. A. Phosphodiesterases and cyclic GMP regulation in heart muscle. Physiology 27, 248-258, doi:10.1152/physiol.00011.2012 (2012).

115 Guazzi, M., Vicenzi, M., Arena, R. \& Guazzi, M. D. PDE5 inhibition with sildenafil improves left ventricular diastolic function, cardiac geometry, and clinical status in patients with stable systolic heart failure: results of a 1-year, prospective, randomized, placebo-controlled study. Circulation. Heart failure 4, 8-17, doi:10.1161/CIRCHEARTFAILURE.110.944694 (2011).

116 Soderling, S. H., Bayuga, S. J. \& Beavo, J. A. Cloning and characterization of a cAMP-specific cyclic nucleotide phosphodiesterase. Proceedings of the National Academy of Sciences of the United States of America 95, 8991-8996 (1998).

117 Fisher, D. A., Smith, J. F., Pillar, J. S., St Denis, S. H. \& Cheng, J. B. Isolation and characterization of PDE8A, a novel human CAMP-specific phosphodiesterase. Biochemical and biophysical research communications 246, 570-577, doi:10.1006/bbrc.1998.8684 (1998).

118 Hayashi, M. et al. Molecular cloning and characterization of human PDE8B, a novel thyroidspecific isozyme of 3',5'-cyclic nucleotide phosphodiesterase. Biochemical and biophysical research communications 250, 751-756, doi:10.1006/bbrc.1998.9379 (1998).

119 Brown, K. M., Lee, L. C., Findlay, J. E., Day, J. P. \& Baillie, G. S. Cyclic AMP-specific phosphodiesterase, PDE8A1, is activated by protein kinase A-mediated phosphorylation. FEBS letters 586, 1631-1637, doi:10.1016/j.febslet.2012.04.033 (2012).

120 Patrucco, E., Albergine, M. S., Santana, L. F. \& Beavo, J. A. Phosphodiesterase 8A (PDE8A) regulates excitation-contraction coupling in ventricular myocytes. Journal of molecular and cellular cardiology 49, 330-333, doi:10.1016/j.yjmcc.2010.03.016 (2010).

121 Fisher, D. A., Smith, J. F., Pillar, J. S., St Denis, S. H. \& Cheng, J. B. Isolation and characterization of PDE9A, a novel human CGMP-specific phosphodiesterase. The Journal of biological chemistry 273, 15559-15564 (1998).

122 Lee, D. I. et al. Phosphodiesterase 9A controls nitric-oxide-independent cGMP and hypertrophic heart disease. Nature 519, 472-476, doi:10.1038/nature14332 (2015).

123 Diviani, D., Dodge-Kafka, K. L., Li, J. \& Kapiloff, M. S. A-kinase anchoring proteins: scaffolding proteins in the heart. American journal of physiology. Heart and circulatory physiology 301, H1742-1753, doi:10.1152/ajpheart.00569.2011 (2011).

124 Perino, A., Ghigo, A., Scott, J. D. \& Hirsch, E. Anchoring proteins as regulators of signaling pathways. Circulation research 111, 482-492, doi:10.1161/CIRCRESAHA.111.262899 (2012).

125 Scott, J. D. \& Santana, L. F. A-kinase anchoring proteins: getting to the heart of the matter. Circulation 121, 1264-1271, doi:10.1161/CIRCULATIONAHA.109.896357 (2010).

126 Troger, J., Moutty, M. C., Skroblin, P. \& Klussmann, E. A-kinase anchoring proteins as potential drug targets. British journal of pharmacology 166, 420-433, doi:10.1111/j.14765381.2011.01796.x (2012).

127 Scott, J. D., Dessauer, C. W. \& Tasken, K. Creating order from chaos: cellular regulation by kinase anchoring. Annual review of pharmacology and toxicology 53, 187-210, doi:10.1146/annurev-pharmtox-011112-140204 (2013).

128 Gao, T. et al. cAMP-dependent regulation of cardiac L-type Ca2+ channels requires membrane targeting of PKA and phosphorylation of channel subunits. Neuron 19, 185-196 (1997). 
129 Nichols, C. B. et al. Sympathetic stimulation of adult cardiomyocytes requires association of AKAP5 with a subpopulation of L-type calcium channels. Circulation research 107, 747-756, doi:10.1161/CIRCRESAHA.109.216127 (2010).

130 Henn, V. et al. Identification of a novel A-kinase anchoring protein 18 isoform and evidence for its role in the vasopressin-induced aquaporin-2 shuttle in renal principal cells. The Journal of biological chemistry 279, 26654-26665, doi:10.1074/jbc.M312835200 (2004).

131 Lygren, B. et al. AKAP complex regulates $\mathrm{Ca} 2+$ re-uptake into heart sarcoplasmic reticulum. EMBO reports 8, 1061-1067, doi:10.1038/sj.embor.7401081 (2007).

132 Marx, S. O. et al. Requirement of a macromolecular signaling complex for beta adrenergic receptor modulation of the KCNQ1-KCNE1 potassium channel. Science 295, 496-499, doi:10.1126/science.1066843 (2002).

133 Terrenoire, C., Houslay, M. D., Baillie, G. S. \& Kass, R. S. The cardiac IKs potassium channel macromolecular complex includes the phosphodiesterase PDE4D3. The Journal of biological chemistry 284, 9140-9146, doi:10.1074/jbc.M805366200 (2009).

134 Kapiloff, M. S. et al. An adenylyl cyclase-mAKAPbeta signaling complex regulates cAMP levels in cardiac myocytes. The Journal of biological chemistry 284, 23540-23546, doi:10.1074/jbc.M109.030072 (2009).

135 Dodge-Kafka, K. L. et al. The protein kinase A anchoring protein mAKAP coordinates two integrated cAMP effector pathways. Nature 437, 574-578, doi:10.1038/nature03966 (2005).

136 Tucci, P. J. Pathophysiological characteristics of the post-myocardial infarction heart failure model in rats. Arquivos brasileiros de cardiologia 96, 420-424 (2011).

137 Kolk, M. V. et al. LAD-ligation: a murine model of myocardial infarction. Journal of visualized experiments : JoVE, doi:10.3791/1438 (2009).

138 Rubin, S. A., Fishbein, M. C. \& Swan, H. J. Compensatory hypertrophy in the heart after myocardial infarction in the rat. Journal of the American College of Cardiology 1, 1435-1441 (1983).

139 Frangogiannis, N. G. Pathophysiology of Myocardial Infarction. Comprehensive Physiology 5, 1841-1875, doi:10.1002/cphy.c150006 (2015).

140 Diwan, A. \& Dorn, G. W., 2nd. Decompensation of cardiac hypertrophy: cellular mechanisms and novel therapeutic targets. Physiology 22, 56-64, doi:10.1152/physiol.00033.2006 (2007).

141 BenedPrognostic significance of plasma norepinephrine in patients with asymptomatic left ventricular dysfunction. SOLVD Regulation of PKA binding to AKAPs in the heart: alterations in human heart failureict, C. R. et al. Prognostic significance of plasma norepinephrine in patients with asymptomatic left ventricular dysfunction. SOLVD Investigators. Circulation 94, 690-697 (1996).

142 Movsesian, M. A. \& Bristow, M. R. Alterations in cAMP-mediated signaling and their role in the pathophysiology of dilated cardiomyopathy. Current topics in developmental biology 68, 25-48, doi:10.1016/S0070-2153(05)68002-7 (2005).

143 Ungerer, M., Bohm, M., Elce, J. S., Erdmann, E. \& Lohse, M. J. Altered expression of betaadrenergic receptor kinase and beta 1-adrenergic receptors in the failing human heart. Circulation 87, 454-463 (1993).

144 Wagner, E. et al. Stimulated emission depletion live-cell super-resolution imaging shows proliferative remodeling of T-tubule membrane structures after myocardial infarction. Circulation research 111, 402-414, doi:10.1161/CIRCRESAHA.112.274530 (2012).

145 Lyon, A. R. et al. Loss of T-tubules and other changes to surface topography in ventricular myocytes from failing human and rat heart. Proceedings of the National Academy of Sciences of the United States of America 106, 6854-6859, doi:10.1073/pnas.0809777106 (2009).

146 Wright, P. T. et al. Caveolin-3 regulates compartmentation of cardiomyocyte beta2adrenergic receptor-mediated CAMP signaling. Journal of molecular and cellular cardiology 67, 38-48, doi:10.1016/j.yjmcc.2013.12.003 (2014).

147 Zakhary, D. R., Moravec, C. S. \& Bond, M. Regulation of PKA binding to AKAPs in the heart: alterations in human heart failure. Circulation 101, 1459-1464 (2000). 
148 McConnell, B. K., Moravec, C. S. \& Bond, M. Troponin I phosphorylation and myofilament calcium sensitivity during decompensated cardiac hypertrophy. The American journal of physiology 274, H385-396 (1998).

149 Zakhary, D. R., Moravec, C. S., Stewart, R. W. \& Bond, M. Protein kinase A (PKA)-dependent troponin-I phosphorylation and PKA regulatory subunits are decreased in human dilated cardiomyopathy. Circulation 99, 505-510 (1999).

150 Marx, S. O. et al. PKA phosphorylation dissociates FKBP12.6 from the calcium release channel (ryanodine receptor): defective regulation in failing hearts. Cell 101, 365-376 (2000).

151 Wehrens, X. H. et al. FKBP12.6 deficiency and defective calcium release channel (ryanodine receptor) function linked to exercise-induced sudden cardiac death. Cell 113, 829-840 (2003).

152 Shan, J. et al. Role of chronic ryanodine receptor phosphorylation in heart failure and betaadrenergic receptor blockade in mice. The Journal of clinical investigation 120, 4375-4387, doi:10.1172/JCl37649 (2010).

153 Pare, G. C. et al. The mAKAP complex participates in the induction of cardiac myocyte hypertrophy by adrenergic receptor signaling. Journal of cell science 118, 5637-5646, doi:10.1242/jcs.02675 (2005).

154 Sprenger, J. U. et al. In vivo model with targeted cAMP biosensor reveals changes in receptor-microdomain communication in cardiac disease. Nature communications 6, 6965, doi:10.1038/ncomms7965 (2015).

155 Mehel, H. et al. Phosphodiesterase-2 is up-regulated in human failing hearts and blunts betaadrenergic responses in cardiomyocytes. Journal of the American College of Cardiology 62, 1596-1606, doi:10.1016/j.jacc.2013.05.057 (2013).

156 Ding, B. et al. Functional role of phosphodiesterase 3 in cardiomyocyte apoptosis: implication in heart failure. Circulation 111, 2469-2476, doi:10.1161/01.CIR.0000165128.39715.87 (2005).

157 Smith, C. J. et al. Development of decompensated dilated cardiomyopathy is associated with decreased gene expression and activity of the milrinone-sensitive CAMP phosphodiesterase PDE3A. Circulation 96, 3116-3123 (1997).

158 Baim, D. S. et al. Evaluation of a new bipyridine inotropic agent--milrinone--in patients with severe congestive heart failure. The New England journal of medicine 309, 748-756, doi:10.1056/NEJM198309293091302 (1983).

159 Packer, M. et al. Effect of oral milrinone on mortality in severe chronic heart failure. The PROMISE Study Research Group. The New England journal of medicine 325, 1468-1475, doi:10.1056/NEJM199111213252103 (1991).

160 Froese, A. \& Nikolaev, V. O. Imaging alterations of cardiomyocyte cAMP microdomains in disease. Frontiers in pharmacology 6, 172, doi:10.3389/fphar.2015.00172 (2015).

161 Skou, J. C. The influence of some cations on an adenosine triphosphatase from peripheral nerves. Biochimica et biophysica acta 23, 394-401 (1957).

162 Blaustein, M. P. Sodium ions, calcium ions, blood pressure regulation, and hypertension: a reassessment and a hypothesis. The American journal of physiology 232, C165-173 (1977).

163 Molitoris, B. A. \& Kinne, R. Ischemia induces surface membrane dysfunction. Mechanism of altered $\mathrm{Na}+$-dependent glucose transport. The Journal of clinical investigation 80, 647-654, doi:10.1172/JCl113117 (1987).

164 Bers, D. M. \& Despa, S. Na+ transport in cardiac myocytes; Implications for excitationcontraction coupling. IUBMB life 61, 215-221, doi:10.1002/iub.163 (2009).

165 Bers, D. M., Barry, W. H. \& Despa, S. Intracellular Na+ regulation in cardiac myocytes. Cardiovascular research 57, 897-912 (2003).

166 Fuller, W. et al. Regulation of the cardiac sodium pump. Cellular and molecular life sciences : CMLS 70, 1357-1380, doi:10.1007/s00018-012-1134-y (2013).

167 Clausen, $\mathrm{T}$. Quantification of $\mathrm{Na}+, \mathrm{K}+$ pumps and their transport rate in skeletal muscle: functional significance. The Journal of general physiology 142, 327-345, doi:10.1085/jgp.201310980 (2013). 
168 Hunt, S. A. et al. 2009 focused update incorporated into the ACC/AHA 2005 Guidelines for the Diagnosis and Management of Heart Failure in Adults: a report of the American College of Cardiology Foundation/American Heart Association Task Force on Practice Guidelines: developed in collaboration with the International Society for Heart and Lung Transplantation. Circulation 119, e391-479, doi:10.1161/CIRCULATIONAHA.109.192065 (2009).

169 Simmerman, H. K. \& Jones, L. R. Phospholamban: protein structure, mechanism of action, and role in cardiac function. Physiological reviews 78, 921-947 (1998).

170 Cheung, J. Y. et al. Phospholemman: a novel cardiac stress protein. Clinical and translational science 3, 189-196, doi:10.1111/j.1752-8062.2010.00213.x (2010).

171 Bossuyt, J., Despa, S., Martin, J. L. \& Bers, D. M. Phospholemman phosphorylation alters its fluorescence resonance energy transfer with the Na/K-ATPase pump. The Journal of biological chemistry 281, 32765-32773, doi:10.1074/jbc.M606254200 (2006).

172 Bibert, S., Roy, S., Schaer, D., Horisberger, J. D. \& Geering, K. Phosphorylation of phospholemman (FXYD1) by protein kinases $\mathrm{A}$ and $\mathrm{C}$ modulates distinct $\mathrm{Na}, \mathrm{K}-\mathrm{ATPase}$ isozymes. The Journal of biological chemistry 283, 476-486, doi:10.1074/jbc.M705830200 (2008).

173 Zhang, X. Q. et al. Phospholemman inhibition of the cardiac $\mathrm{Na}+/ \mathrm{Ca} 2+$ exchanger. Role of phosphorylation. The Journal of biological chemistry 281, 7784-7792, doi:10.1074/jbc.M512092200 (2006).

174 Fuller, W. et al. FXYD1 phosphorylation in vitro and in adult rat cardiac myocytes: threonine 69 is a novel substrate for protein kinase C. American journal of physiology. Cell physiology 296, C1346-1355, doi:10.1152/ajpcell.00523.2008 (2009).

175 Morth, J. P. et al. Crystal structure of the sodium-potassium pump. Nature 450, 1043-1049, doi:10.1038/nature06419 (2007).

176 Shinoda, T., Ogawa, H., Cornelius, F. \& Toyoshima, C. Crystal structure of the sodiumpotassium pump at 2.4 A resolution. Nature 459, 446-450, doi:10.1038/nature07939 (2009).

177 Ogawa, H., Shinoda, T., Cornelius, F. \& Toyoshima, C. Crystal structure of the sodiumpotassium pump ( $\mathrm{Na}+, \mathrm{K}+-\mathrm{ATPase}$ ) with bound potassium and ouabain. Proceedings of the National Academy of Sciences of the United States of America 106, 13742-13747, doi:10.1073/pnas.0907054106 (2009).

178 Kaplan, J. H. Biochemistry of Na,K-ATPase. Annual review of biochemistry 71, 511-535, doi:10.1146/annurev.biochem.71.102201.141218 (2002).

179 Sweadner, K. J. Isozymes of the Na+/K+-ATPase. Biochimica et biophysica acta 988, 185-220 (1989).

180 Geering, K. The functional role of the beta-subunit in the maturation and intracellular transport of Na,K-ATPase. FEBS letters 285, 189-193 (1991).

181 Sweadner, K. J. \& Rael, E. The FXYD gene family of small ion transport regulators or channels: cDNA sequence, protein signature sequence, and expression. Genomics 68, 41-56, doi:10.1006/geno.2000.6274 (2000).

182 Cornelius, F. \& Mahmmoud, Y. A. Functional modulation of the sodium pump: the regulatory proteins "Fixit". News in physiological sciences : an international journal of physiology produced jointly by the International Union of Physiological Sciences and the American Physiological Society 18, 119-124 (2003).

183 Horisberger, J. D., Jaunin, P., Good, P. J., Rossier, B. C. \& Geering, K. Coexpression of alpha 1 with putative beta 3 subunits results in functional $\mathrm{Na}+/ \mathrm{K}+$ pumps in Xenopus oocytes.

Proceedings of the National Academy of Sciences of the United States of America 88, 83978400 (1991).

184 Berry, R. G., Despa, S., Fuller, W., Bers, D. M. \& Shattock, M. J. Differential distribution and regulation of mouse cardiac $\mathrm{Na}+/ \mathrm{K}+-\mathrm{ATPase}$ alpha1 and alpha2 subunits in T-tubule and surface sarcolemmal membranes. Cardiovascular research 73, 92-100, doi:10.1016/j.cardiores.2006.11.006 (2007). 
185 Swift, F., Tovsrud, N., Enger, U. H., Sjaastad, I. \& Sejersted, O. M. The Na+/K+-ATPase alpha2isoform regulates cardiac contractility in rat cardiomyocytes. Cardiovascular research 75 , 109-117, doi:10.1016/j.cardiores.2007.03.017 (2007).

186 Swift, F. et al. Altered $\mathrm{Na}+/ \mathrm{Ca2+-exchanger} \mathrm{activity} \mathrm{due} \mathrm{to} \mathrm{downregulation} \mathrm{of} \mathrm{Na}+/ \mathrm{K}+-\mathrm{ATPase}$ alpha2-isoform in heart failure. Cardiovascular research 78, 71-78, doi:10.1093/cvr/cvn013 (2008).

187 Dostanic, I., Schultz Jel, J., Lorenz, J. N. \& Lingrel, J. B. The alpha 1 isoform of Na,K-ATPase regulates cardiac contractility and functionally interacts and co-localizes with the $\mathrm{Na} / \mathrm{Ca}$ exchanger in heart. The Journal of biological chemistry 279, 54053-54061, doi:10.1074/jbc.M410737200 (2004).

188 Pavlovic, D., Fuller, W. \& Shattock, M. J. Novel regulation of cardiac Na pump via phospholemman. Journal of molecular and cellular cardiology 61, 83-93, doi:10.1016/j.yjmcc.2013.05.002 (2013).

189 Despa, S., Lingrel, J. B. \& Bers, D. M. Na(+)/K)+)-ATPase alpha2-isoform preferentially modulates $\mathrm{Ca} 2(+)$ transients and sarcoplasmic reticulum $\mathrm{Ca} 2(+)$ release in cardiac myocytes. Cardiovascular research 95, 480-486, doi:10.1093/cvr/cvs213 (2012).

190 Shattock, M. J. et al. Na+/Ca2+ exchange and $\mathrm{Na}+/ \mathrm{K}+-\mathrm{ATP}$ ase in the heart. The Journal of physiology 593, 1361-1382, doi:10.1113/jphysiol.2014.282319 (2015).

191 James, P. F. et al. Identification of a specific role for the Na,K-ATPase alpha 2 isoform as a regulator of calcium in the heart. Molecular cell 3, 555-563 (1999).

192 Tian, J. \& Xie, Z. J. The Na-K-ATPase and calcium-signaling microdomains. Physiology 23, 205211, doi:10.1152/physiol.00008.2008 (2008).

193 Liu, L., Zhao, X., Pierre, S. V. \& Askari, A. Association of PI3K-Akt signaling pathway with digitalis-induced hypertrophy of cardiac myocytes. American journal of physiology. Cell physiology 293, C1489-1497, doi:10.1152/ajpcell.00158.2007 (2007).

194 Geering, K. FXYD proteins: new regulators of Na-K-ATPase. American journal of physiology. Renal physiology 290, F241-250, doi:10.1152/ajprenal.00126.2005 (2006).

195 Palmer, C. J., Scott, B. T. \& Jones, L. R. Purification and complete sequence determination of the major plasma membrane substrate for CAMP-dependent protein kinase and protein kinase C in myocardium. The Journal of biological chemistry 266, 11126-11130 (1991).

196 Bogaev, R. C. et al. Gene structure and expression of phospholemman in mouse. Gene 271, 69-79 (2001).

197 Wetzel, R. K. \& Sweadner, K. J. Phospholemman expression in extraglomerular mesangium and afferent arteriole of the juxtaglomerular apparatus. American journal of physiology. Renal physiology 285, F121-129, doi:10.1152/ajprenal.00241.2002 (2003).

198 Feschenko, M. S. et al. Phospholemman, a single-span membrane protein, is an accessory protein of $\mathrm{Na}, \mathrm{K}-\mathrm{ATP}$ ase in cerebellum and choroid plexus. The Journal of neuroscience : the official journal of the Society for Neuroscience 23, 2161-2169 (2003).

199 Walaas, S. I., Czernik, A. J., Olstad, O. K., Sletten, K. \& Walaas, O. Protein kinase C and cyclic AMP-dependent protein kinase phosphorylate phospholemman, an insulin and adrenalineregulated membrane phosphoprotein, at specific sites in the carboxy terminal domain. The Biochemical journal 304 ( Pt 2), 635-640 (1994).

200 Han, F., Bossuyt, J., Despa, S., Tucker, A. L. \& Bers, D. M. Phospholemman phosphorylation mediates the protein kinase $\mathrm{C}$-dependent effects on $\mathrm{Na}+/ \mathrm{K}+$ pump function in cardiac myocytes. Circulation research 99, 1376-1383, doi:10.1161/01.RES.0000251667.73461.fb (2006).

201 Khafaga, M. et al. $\mathrm{Na}(+) / \mathrm{K}(+)$-ATPase E960 and phospholemman F28 are critical for their functional interaction. Proceedings of the National Academy of Sciences of the United States of America 109, 20756-20761, doi:10.1073/pnas.1207866109 (2012).

202 Pavlovic, D., Fuller, W. \& Shattock, M. J. The intracellular region of FXYD1 is sufficient to regulate cardiac $\mathrm{Na} / \mathrm{K}$ ATPase. FASEB journal : official publication of the Federation of 
American Societies for Experimental Biology 21, 1539-1546, doi:10.1096/fj.06-7269com (2007).

203 Silverman, B. et al. Serine 68 phosphorylation of phospholemman: acute isoform-specific activation of cardiac Na/K ATPase. Cardiovascular research 65, 93-103, doi:10.1016/j.cardiores.2004.09.005 (2005).

204 Zhang, X. Q. et al. Phospholemman overexpression inhibits Na+-K+-ATPase in adult rat cardiac myocytes: relevance to decreased $\mathrm{Na}+$ pump activity in postinfarction myocytes. Journal of applied physiology 100, 212-220, doi:10.1152/japplphysiol.00757.2005 (2006).

205 Bossuyt, J. et al. Isoform specificity of the $\mathrm{Na}$ /K-ATPase association and regulation by phospholemman. The Journal of biological chemistry 284, 26749-26757, doi:10.1074/jbc.M109.047357 (2009).

206 Lifshitz, Y., Lindzen, M., Garty, H. \& Karlish, S. J. Functional interactions of phospholemman (PLM) (FXYD1) with Na+,K+-ATPase. Purification of alpha1/beta1/PLM complexes expressed in Pichia pastoris. The Journal of biological chemistry 281, 15790-15799, doi:10.1074/jbc.M601993200 (2006).

207 Wang, X. et al. Phospholemman modulates the gating of cardiac L-type calcium channels. Biophysical journal 98, 1149-1159, doi:10.1016/j.bpj.2009.11.032 (2010).

208 Moorman, J. R. et al. Unitary anion currents through phospholemman channel molecules. Nature 377, 737-740, doi:10.1038/377737a0 (1995).

209 Moorman, J. R., Palmer, C. J., John, J. E., 3rd, Durieux, M. E. \& Jones, L. R. Phospholemman expression induces a hyperpolarization-activated chloride current in Xenopus oocytes. The Journal of biological chemistry 267, 14551-14554 (1992).

210 Morales-Mulia, M., Pasantes-Morales, H. \& Moran, J. Volume sensitive efflux of taurine in HEK293 cells overexpressing phospholemman. Biochimica et biophysica acta 1496, 252-260 (2000).

211 Despa, S., Tucker, A. L. \& Bers, D. M. Phospholemman-mediated activation of Na/K-ATPase limits [Na]i and inotropic state during beta-adrenergic stimulation in mouse ventricular myocytes. Circulation 117, 1849-1855, doi:10.1161/CIRCULATIONAHA.107.754051 (2008).

212 Wang, J. et al. Phospholemman and beta-adrenergic stimulation in the heart. American journal of physiology. Heart and circulatory physiology 298, H807-815, doi:10.1152/ajpheart.00877.2009 (2010).

213 Presti, C. F., Jones, L. R. \& Lindemann, J. P. Isoproterenol-induced phosphorylation of a 15kilodalton sarcolemmal protein in intact myocardium. The Journal of biological chemistry 260, 3860-3867 (1985).

214 Presti, C. F., Scott, B. T. \& Jones, L. R. Identification of an endogenous protein kinase C activity and its intrinsic 15-kilodalton substrate in purified canine cardiac sarcolemmal vesicles. The Journal of biological chemistry 260, 13879-13889 (1985).

215 Sweadner, K. J. \& Feschenko, M. S. Predicted location and limited accessibility of protein kinase A phosphorylation site on Na-K-ATPase. American journal of physiology. Cell physiology 280, C1017-1026 (2001).

216 Bell, J. R. et al. Characterization of the phospholemman knockout mouse heart: depressed left ventricular function with increased Na-K-ATPase activity. American journal of physiology. Heart and circulatory physiology 294, H613-621, doi:10.1152/ajpheart.01332.2007 (2008).

217 Bossuyt, J., Ai, X., Moorman, J. R., Pogwizd, S. M. \& Bers, D. M. Expression and phosphorylation of the na-pump regulatory subunit phospholemman in heart failure. Circulation research 97, 558-565, doi:10.1161/01.RES.0000181172.27931.c3 (2005).

218 Fuller, W., Eaton, P., Bell, J. R. \& Shattock, M. J. Ischemia-induced phosphorylation of phospholemman directly activates rat cardiac Na/K-ATPase. FASEB journal : official publication of the Federation of American Societies for Experimental Biology 18, 197-199, doi:10.1096/fj.03-0213fje (2004). 
219 Pavlovic, D. et al. Nitric oxide regulates cardiac intracellular $\mathrm{Na}(+)$ and $\mathrm{Ca}(2)(+)$ by modulating $\mathrm{Na} / \mathrm{K}$ ATPase via PKCepsilon and phospholemman-dependent mechanism. Journal of molecular and cellular cardiology 61, 164-171, doi:10.1016/j.yjmcc.2013.04.013 (2013).

220 Song, J. et al. Serine 68 of phospholemman is critical in modulation of contractility, [Ca2+]i transients, and $\mathrm{Na}+/ \mathrm{Ca} 2+$ exchange in adult rat cardiac myocytes. American journal of physiology. Heart and circulatory physiology 288, H2342-2354, doi:10.1152/ajpheart.01133.2004 (2005).

221 Wang, J. et al. Regulation of in vivo cardiac contractility by phospholemman: role of $\mathrm{Na}+/ \mathrm{Ca} 2+$ exchange. American journal of physiology. Heart and circulatory physiology 300, H859-868, doi:10.1152/ajpheart.00894.2010 (2011).

222 Neubauer, S., Newell, J. B. \& Ingwall, J. S. Metabolic consequences and predictability of ventricular fibrillation in hypoxia. A 31P- and 23Na-nuclear magnetic resonance study of the isolated rat heart. Circulation 86, 302-310 (1992).

223 Tani, M. \& Neely, J. R. Role of intracellular $\mathrm{Na}+$ in Ca2+ overload and depressed recovery of ventricular function of reperfused ischemic rat hearts. Possible involvement of $\mathrm{H}+-\mathrm{Na}+$ and $\mathrm{Na}+-\mathrm{Ca} 2+$ exchange. Circulation research 65, 1045-1056 (1989).

224 Pogwizd, S. M., Sipido, K. R., Verdonck, F. \& Bers, D. M. Intracellular Na in animal models of hypertrophy and heart failure: contractile function and arrhythmogenesis. Cardiovascular research 57, 887-896 (2003).

225 Verdonck, F., Volders, P. G., Vos, M. A. \& Sipido, K. R. Intracellular Na+ and altered Na+ transport mechanisms in cardiac hypertrophy and failure. Journal of molecular and cellular cardiology 35, 5-25 (2003).

226 Verdonck, F., Volders, P. G., Vos, M. A. \& Sipido, K. R. Increased Na+ concentration and altered $\mathrm{Na} / \mathrm{K}$ pump activity in hypertrophied canine ventricular cells. Cardiovascular research 57, 1035-1043 (2003).

227 Despa, S., Islam, M. A., Weber, C. R., Pogwizd, S. M. \& Bers, D. M. Intracellular Na(+) concentration is elevated in heart failure but $\mathrm{Na} / \mathrm{K}$ pump function is unchanged. Circulation 105, 2543-2548 (2002).

228 Undrovinas, A. I., Maltsev, V. A. \& Sabbah, H. N. Repolarization abnormalities in cardiomyocytes of dogs with chronic heart failure: role of sustained inward current. Cellular and molecular life sciences: CMLS 55, 494-505 (1999).

229 Fuller, W., Parmar, V., Eaton, P., Bell, J. R. \& Shattock, M. J. Cardiac ischemia causes inhibition of the $\mathrm{Na} / \mathrm{K}$ ATPase by a labile cytosolic compound whose production is linked to oxidant stress. Cardiovascular research 57, 1044-1051 (2003).

230 Boguslavskyi, A. et al. Cardiac hypertrophy in mice expressing unphosphorylatable phospholemman. Cardiovascular research 104, 72-82, doi:10.1093/cvr/cvu182 (2014).

231 El-Armouche, A. et al. Phospholemman-dependent regulation of the cardiac Na/K-ATPase activity is modulated by inhibitor-1 sensitive type-1 phosphatase. FASEB journal : official publication of the Federation of American Societies for Experimental Biology 25, 4467-4475, doi:10.1096/fj.11-184903 (2011).

232 Semb, S. O. et al. Reduced myocardial $\mathrm{Na}+, \mathrm{K}(+)$-pump capacity in congestive heart failure following myocardial infarction in rats. Journal of molecular and cellular cardiology 30, 13111328 (1998).

233 Allen, P. D., Schmidt, T. A., Marsh, J. D. \& Kjeldsen, K. Na,K-ATPase expression in normal and failing human left ventricle. Basic research in cardiology 87 Suppl 1, 87-94 (1992).

234 Mirza, M. A. et al. Phospholemman deficiency in postinfarct hearts: enhanced contractility but increased mortality. Clinical and translational science 5, 235-242, doi:10.1111/j.17528062.2012.00403.x (2012).

235 Correll, R. N. et al. Overexpression of the $\mathrm{Na}+/ \mathrm{K}+$ ATPase alpha2 but not alpha1 isoform attenuates pathological cardiac hypertrophy and remodeling. Circulation research 114, 249256, doi:10.1161/CIRCRESAHA.114.302293 (2014). 
236 Brooker, G., Harper, J. F., Terasaki, W. L. \& Moylan, R. D. Radioimmunoassay of cyclic AMP and cyclic GMP. Advances in cyclic nucleotide research 10, 1-33 (1979).

237 Harper, J. F. \& Brooker, G. Femtomole sensitive radioimmunoassay for cyclic AMP and cyclic GMP after 2' 0 acetylation by acetic anhydride in aqueous solution. Journal of cyclic nucleotide research 1, 207-218 (1975).

238 Williams, C. cAMP detection methods in HTS: selecting the best from the rest. Nature reviews. Drug discovery 3, 125-135, doi:10.1038/nrd1306 (2004).

239 Sprenger, J. U. \& Nikolaev, V. O. Biophysical techniques for detection of cAMP and cGMP in living cells. International journal of molecular sciences 14, 8025-8046, doi:10.3390/ijms14048025 (2013).

240 Förster, T. Zwischenmolekulare Energiewanderung und Fluoreszenz. Annalen der Physik 437, 55-75, doi:10.1002/andp.19484370105 (1948).

241 Piston, D. W. \& Kremers, G. J. Fluorescent protein FRET: the good, the bad and the ugly. Trends in biochemical sciences 32, 407-414, doi:10.1016/j.tibs.2007.08.003 (2007).

242 Shrestha, D., Jenei, A., Nagy, P., Vereb, G. \& Szollosi, J. Understanding FRET as a research tool for cellular studies. International journal of molecular sciences 16, 6718-6756, doi:10.3390/ijms16046718 (2015).

243 Zhang, J., Campbell, R. E., Ting, A. Y. \& Tsien, R. Y. Creating new fluorescent probes for cell biology. Nature reviews. Molecular cell biology 3, 906-918, doi:10.1038/nrm976 (2002).

244 Frings, S., Seifert, R., Godde, M. \& Kaupp, U. B. Profoundly different calcium permeation and blockage determine the specific function of distinct cyclic nucleotide-gated channels. Neuron 15, 169-179 (1995).

245 Willoughby, D. \& Cooper, D. M. Organization and Ca2+ regulation of adenylyl cyclases in cAMP microdomains. Physiological reviews 87, 965-1010, doi:10.1152/physrev.00049.2006 (2007).

246 Rochais, F. et al. Negative feedback exerted by cAMP-dependent protein kinase and cAMP phosphodiesterase on subsarcolemmal cAMP signals in intact cardiac myocytes: an in vivo study using adenovirus-mediated expression of CNG channels. The Journal of biological chemistry 279, 52095-52105, doi:10.1074/jbc.M405697200 (2004).

247 Adams, S. R., Harootunian, A. T., Buechler, Y. J., Taylor, S. S. \& Tsien, R. Y. Fluorescence ratio imaging of cyclic AMP in single cells. Nature 349, 694-697, doi:10.1038/349694a0 (1991).

248 Zaccolo, M. et al. A genetically encoded, fluorescent indicator for cyclic AMP in living cells. Nature cell biology 2, 25-29, doi:10.1038/71345 (2000).

249 Nikolaev, V. O., Bunemann, M., Hein, L., Hannawacker, A. \& Lohse, M. J. Novel single chain cAMP sensors for receptor-induced signal propagation. The Journal of biological chemistry 279, 37215-37218, doi:10.1074/jbc.C400302200 (2004).

250 Nikolaev, V. O., Gambaryan, S., Engelhardt, S., Walter, U. \& Lohse, M. J. Real-time monitoring of the PDE2 activity of live cells: hormone-stimulated cAMP hydrolysis is faster than hormone-stimulated CAMP synthesis. The Journal of biological chemistry 280, 1716-1719, doi:10.1074/jbc.C400505200 (2005).

251 Perera, R. K. et al. Microdomain switch of cGMP-regulated phosphodiesterases leads to ANPinduced augmentation of beta-adrenoceptor-stimulated contractility in early cardiac hypertrophy. Circulation research 116, 1304-1311, doi:10.1161/CIRCRESAHA.116.306082 (2015).

252 Howie, J. et al. Substrate recognition by the cell surface palmitoyl transferase DHHC5. Proceedings of the National Academy of Sciences of the United States of America 111, 1753417539, doi:10.1073/pnas.1413627111 (2014).

253 Lewis, C. J., Gong, H., Brown, M. J. \& Harding, S. E. Overexpression of beta 1-adrenoceptors in adult rat ventricular myocytes enhances CGP 12177A cardiostimulation: implications for 'putative' beta 4-adrenoceptor pharmacology. British journal of pharmacology 141, 813-824, doi:10.1038/sj.bjp.0705668 (2004). 
254 Tulloch, L. B. et al. The inhibitory effect of phospholemman on the sodium pump requires its palmitoylation. The Journal of biological chemistry 286, 36020-36031, doi:10.1074/jbc.M111.282145 (2011).

255 Jia, L. G. et al. Hypertrophy, increased ejection fraction, and reduced Na-K-ATPase activity in phospholemman-deficient mice. American journal of physiology. Heart and circulatory physiology 288, H1982-1988, doi:10.1152/ajpheart.00142.2004 (2005).

256 Chen, L. S., Lo, C. F., Numann, R. \& Cuddy, M. Characterization of the human and rat phospholemman (PLM) CDNAs and localization of the human PLM gene to chromosome 19q13.1. Genomics 41, 435-443, doi:10.1006/geno.1997.4665 (1997).

257 Louch, W. E., Sheehan, K. A. \& Wolska, B. M. Methods in cardiomyocyte isolation, culture, and gene transfer. Journal of molecular and cellular cardiology 51, 288-298, doi:10.1016/j.yjmcc.2011.06.012 (2011).

258 Calebiro, D. et al. Persistent cAMP-signals triggered by internalized G-protein-coupled receptors. PLoS biology 7, e1000172, doi:10.1371/journal.pbio.1000172 (2009).

259 Lomas, O. et al. Adenoviral transduction of FRET-based biosensors for CAMP in primary adult mouse cardiomyocytes. Methods in molecular biology 1294, 103-115, doi:10.1007/978-14939-2537-7_8 (2015).

260 Leroy, J. et al. Spatiotemporal dynamics of beta-adrenergic cAMP signals and L-type Ca2+ channel regulation in adult rat ventricular myocytes: role of phosphodiesterases. Circulation research 102, 1091-1100, doi:10.1161/CIRCRESAHA.107.167817 (2008).

261 Mika, D., Richter, W., Westenbroek, R. E., Catterall, W. A. \& Conti, M. PDE4B mediates local feedback regulation of beta(1)-adrenergic cAMP signaling in a sarcolemmal compartment of cardiac myocytes. Journal of cell science 127, 1033-1042, doi:10.1242/jcs.140251 (2014).

262 Shattock, M. J. Phospholemman: its role in normal cardiac physiology and potential as a druggable target in disease. Current opinion in pharmacology 9, 160-166, doi:10.1016/j.coph.2008.12.015 (2009).

263 Fischmeister, R., Castro, L., Abi-Gerges, A., Rochais, F. \& Vandecasteele, G. Species- and tissue-dependent effects of NO and cyclic GMP on cardiac ion channels. Comparative biochemistry and physiology. Part A, Molecular \& integrative physiology 142, 136-143, doi:10.1016/j.cbpb.2005.04.012 (2005).

264 Rivet-Bastide, M. et al. cGMP-stimulated cyclic nucleotide phosphodiesterase regulates the basal calcium current in human atrial myocytes. The Journal of clinical investigation 99, 27102718, doi:10.1172/JCl119460 (1997).

265 Madhani, M. et al. Phospholemman Ser69 phosphorylation contributes to sildenafil-induced cardioprotection against reperfusion injury. American journal of physiology. Heart and circulatory physiology 299, H827-836, doi:10.1152/ajpheart.00129.2010 (2010).

266 Liu, L. \& Askari, A. Beta-subunit of cardiac Na+-K+-ATPase dictates the concentration of the functional enzyme in caveolae. American journal of physiology. Cell physiology 291, C569578, doi:10.1152/ajpcell.00002.2006 (2006).

267 Liu, L. et al. Role of caveolae in signal-transducing function of cardiac Na+/K+-ATPase. American journal of physiology. Cell physiology 284, C1550-1560, doi:10.1152/ajpcell.00555.2002 (2003).

268 Tian, J., Gong, X. \& Xie, Z. Signal-transducing function of Na+-K+-ATPase is essential for ouabain's effect on [Ca2+]i in rat cardiac myocytes. American journal of physiology. Heart and circulatory physiology 281, H1899-1907 (2001).

269 Warrier, S. et al. Beta-adrenergic- and muscarinic receptor-induced changes in cAMP activity in adult cardiac myocytes detected with FRET-based biosensor. American journal of physiology. Cell physiology 289, C455-461, doi:10.1152/ajpcell.00058.2005 (2005).

270 Horackova, M. \& Byczko, Z. Differences in the structural characteristics of adult guinea pig and rat cardiomyocytes during their adaptation and maintenance in long-term cultures: confocal microscopy study. Experimental cell research 237, 158-175, doi:10.1006/excr.1997.3775 (1997). 
271 Xiang, Y. K. Compartmentalization of beta-adrenergic signals in cardiomyocytes. Circulation research 109, 231-244, doi:10.1161/CIRCRESAHA.110.231340 (2011).

272 Baillie, G. S. et al. beta-Arrestin-mediated PDE4 cAMP phosphodiesterase recruitment regulates beta-adrenoceptor switching from Gs to Gi. Proceedings of the National Academy of Sciences of the United States of America 100, 940-945, doi:10.1073/pnas.262787199 (2003).

273 Beca, S. et al. Phosphodiesterase type 3A regulates basal myocardial contractility through interacting with sarcoplasmic reticulum calcium ATPase type 2a signaling complexes in mouse heart. Circulation research 112, 289-297, doi:10.1161/CIRCRESAHA.111.300003 (2013).

274 Malecot, C. O., Bers, D. M. \& Katzung, B. G. Biphasic contractions induced by milrinone at low temperature in ferret ventricular muscle: role of the sarcoplasmic reticulum and transmembrane calcium influx. Circulation research 59, 151-162 (1986).

275 Jurevicius, J., Skeberdis, V. A. \& Fischmeister, R. Role of cyclic nucleotide phosphodiesterase isoforms in cAMP compartmentation following beta2-adrenergic stimulation of $\mathrm{ICa}, \mathrm{L}$ in frog ventricular myocytes. The Journal of physiology 551, 239-252, doi:10.1113/jphysiol.2003.045211 (2003).

276 Yan, C., Miller, C. L. \& Abe, J. Regulation of phosphodiesterase 3 and inducible cAMP early repressor in the heart. Circulation research 100, 489-501, doi:10.1161/01.RES.0000258451.44949.d7 (2007).

277 Lohmann, S. M., Fischmeister, R. \& Walter, U. Signal transduction by cGMP in heart. Basic research in cardiology 86, 503-514 (1991).

278 Zoccarato, A. et al. Cardiac Hypertrophy Is Inhibited by a Local Pool of cAMP Regulated by Phosphodiesterase 2. Circulation research 117, 707-719, doi:10.1161/CIRCRESAHA.114.305892 (2015).

279 Song, J. et al. Overexpression of phospholemman alters contractility and [Ca(2+)](i) transients in adult rat myocytes. American journal of physiology. Heart and circulatory physiology $\mathbf{2 8 3}$ H576-583, doi:10.1152/ajpheart.00197.2002 (2002). 


\section{Acknowledgements}

It was a great honor and absolute pleasure to work with my supervisor and excellent guide Prof. Viacheslav Nikolaev, who supported and encouraged me giving best strategies throughout this exciting project. I would also like to thank him for giving me opportunities to develop my own ideas and motivating me with alternative experimental approaches. I very much appreciate having you as mentor during the past three years, Slava.

I would like to express my sincere gratitude to all members of my thesis committee, Prof. Blanche Schwappach, Prof. Walter Stühmer and Prof. Michael Shattock for inspiring thoughts about my work and vivid discussions in the time of our committee meetings. I am very grateful to Prof. Dörthe Katschinski and Prof. Susanne Lutz for accepting to be on my examination board and commenting on my thesis.

Part of the ideas for this dissertation stem from my co-supervisor Prof. Michael Shattock, whose enthusiasm always gave me the strength at the right time and made me learn and profit from my mistakes. It was an extremely enriching experience to work with him and his colleagues. I would like to address my genuine gratitude to him, Dr. Davor Pavlovic and Dr. Andrii Boguslavskyi at the Rayne Institute for all the support through the 'London-phase' of my project. At this point, I would also like to extend many thanks to Dr. William Fuller and Dr. Jacqueline Howie for their advice and assistance in generating the Rb-data in Dundee.

I am gratefully indebt to Prof. Julia Gorelik and Dr. Peter Wright for their cooperation and huge help in producing valuable MI-data.

I owe very special thanks to my teammates; Karina Schlosser for excellent technical support and always an open ear for me, as well as Tobias Goldak without whose help most of the experiments would not be possible. Julia Sprenger, Ruwan Perera and Konrad Götz were the best colleagues and friends one could imagine during my PhD time, thank you all for warmly welcoming me and helping me in all kinds of problems.

I surely want to thank the 'IRTG1816 family' and all its members, the administrative staff as well as each student. We shared so many unforgettable moments during the retreats, lunches and numerous events which gave me much more reason to the think that 'research can be fun'. Therefore I would like to thank especially Prof. Dörthe Katschinski for making the IRTG possible.

Finally, I would like to say that I am extremely lucky in having such wonderful parents and sisters who were so patient and have supported me in every way possible. I would like to thank my family and my husband Mahmut for their endless encouragements and for making every thesis related life circumstance easier. 